\title{
Champs affines
}

\section{Bertrand Toën}

\begin{abstract}
The purpose of this work is to introduce a notion of affine stacks, which is a homotopy version of the notion of affine schemes, and to give several applications in the context of algebraic topology and algebraic geometry.

As a first application we show how affine stacks can be used in order to give a new point of view (and new proofs) on rational and $p$-adic homotopy theory. This gives a first solution to A. Grothendieck's schematization problem described in [18].

We also use affine stacks in order to introduce a notion of schematic homotopy types. We show that schematic homotopy types give a second solution to the schematization problem, which also allows us to go beyound rational and $p$-adic homotopy theory for spaces with arbitrary fundamental groups. The notion of schematic homotopy types is also used in order to construct various homotopy types of algebraic varieties corresponding to various cohomology theories (Betti, de Rham, $l$-adic, ...), extending the well known constructions of the various fundamental groups.

Finally, as algebraic stacks are obtained by gluing affine schemes we define $\infty$-geometric stacks as a certain gluing of affine stacks. Example of $\infty$ geometric stacks in the context of algebraic topology (moduli spaces of dga structures up to quasi-isomorphisms) and Hodge theory (non-abelian periods) are given.
\end{abstract}

Mathematics Subject Classification (2000). 14F25.

Keywords. Stacks, schematic homotopy type, rational and p-adic homotopy theory. 
Table des matières

0. Introduction 3

1. Rappels sur les champs $\quad 15$

1.1. Rappel des définitions $\quad 15$

1.2. Limites homotopiques et décomposition de Postnikov 18

1.3. Cohomologie des préfaisceaux simpliciaux 23

1.4. $H_{\infty}$-Champs 28

1.5. Schémas en groupes affines 31

2. Champs affines $\quad 35$

2.1. Rappel sur les algèbres co-simpliciales 36

2.2. Champs affines 41

2.3. Affination des types d'homotopie 48

2.4. Champs affines sur un corps 51

2.5. Comparaison avec l'homotopie rationnelle et $p$-adique 61

2.6. Critique des champs affines 65

3. $\infty$-Gerbes affines et types d'homotopie schématiques 65

$\begin{array}{lll}\text { 3.1. Définitions } & 66\end{array}$

3.2. Exemples d' $\infty$-gerbes affines et de types d'homotopie schématiques 70

3.3. Schématisation des types d'homotopie 74

$\begin{array}{ll}\text { 3.4. Exemples et contre-exemples } & 79\end{array}$

3.5. Types d'homotopie des variétés algébriques 82

3.5.1. Type d'homotopie schématiques et théorie de Hodge 82

3.5.2. Type d'homotopie (iso-)cristallin 85

\begin{tabular}{ll} 
3.5.3. & Type d'homotopie $l$-adique \\
\hline
\end{tabular}

4. Champs $\infty$-géométriques 89

4.1. Définition 89

4.2. Application $\quad 92$

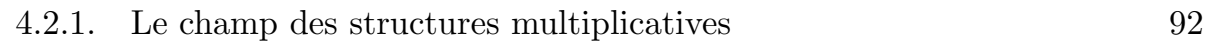

$\begin{array}{lr}\text { 4.2.2. Périodes non-abéliennes } & 96\end{array}$

Appendix A. Le problème de la schématisation 100

$\begin{array}{ll}\text { Références } & 102\end{array}$ 


\section{Introduction}

Le but principal de ce travail est de présenter une notion de champ ${ }^{1}$ affine, généralisation de nature homotopique de la notion de schéma affine, et d'en donner plusieurs applications que nous allons commencer par résumer.

- Dans un premier temps on montrera comment la notion de champs affines peut-être utilisée afin de réinterpréter géométriquement, et d'éclairer, les théories de l'homotopie rationnelle et $p$-adique (par exemple au sens de $[41,3,4,56,34,16,37])$. Ceci passe par l'existence d'un foncteur d'affination, associant à tout type d'homotopie un champ affine vérifiant une propriété universelle. Nous proposerons cette construction comme une première solution au problème de la schématisation de A. Grothendieck de [18] (voir aussi l'appendice $A$ ).

- Les champs affines seront utilisés pour définir une notion de type d'homotopie schématique que nous proposons comme modèles pour construire des types d'homotopie de variétés algébriques. Nous démontrerons l'existence d'un foncteur de schématisation, associant à un type d'homotopie (au sens usuel) un type d'homotopie schématique vérifiant une propriété universelle. Cette construction donne une seconde solution au problème de la schématisation, et permet de plus d'aller au-delà des théories de l'homotopie rationnelle et $p$ adique pour des espaces à groupes fondamentaux arbitraires.

- On construit, pour les théories cohomologiques usuelles de la géométrie algébrique (Betti, de Rham, Hodge, $l$-adique et cristalline) des théories homotopiques associées dont les valeurs sont des types d'homotopie schématiques. On espère que ces théories d'homotopie capturent des informations arithmétiques et/ou géométriques intéressantes. Dans le cas des variétés complexes projectives et de la théorie de Betti, on explique comment le type d'homotopie associé possède une décomposition de Hodge généralisant les structures de Hodge sur la coholomogie, le groupe fondamental et sur le type d'homotopie rationnel. De même, dans le cas de la cohomologie cristalline le type d'homotopie associé possède une structure de $F$-isocristal (due a M. Olsson, voir $[40]$ ), et pour le cas de la cohomologie $l$-adique une action continue du groupe de Galois du corps de base. On propose aussi une construction d'une variante non-abélienne des applications d'Abel Jacobi.

- Tout comme un schéma (ou plus généralement un champ algébrique) est obtenu par recollement de schémas affines, on peut recoller des champs affines pour obtenir une notion de champs $\infty$-géométriques. Cette notion, qui est une généralisation de la notion de champs algébriques, permet de résoudre de nouveaux problèmes de modules qui ne peuvent être raisonnablement résolus à l'aide de la notion usuelle de champs algébriques. On construit par exemple le champ $\infty$-géométrique qui classifie les structures d'algèbre

\footnotetext{
${ }^{1}$ Dans ce texte l'expression champ signiefira toujours champ en $\infty$-groupoides. Un modèle pour la théorie des champs (que nous utiliserons) est celui des préfaisceaux simpliciaux de A. Joyal et R. Jardine (voir [30, 28]).
} 
différentielle graduée à quasi-isomorphismes près. On construit aussi le champ $\infty$-géométrique classifiant les filtrations sur une algèbre différentielle graduée commutative, que l'on utilise pour définir une application des périodes nonabéliennes qui contrôle la variation de la filtration de Hodge sur le type d'homotopie rationnel d'une famille de variétés complexes lisses et projectives.

Certains des points précédents seront traités en détails, mais d'autres feront l'objet d'articles à parts entières et ne seront que survolés dans ce travail.

Décrivons à présent le contenu mathématique de cet article.

\section{Champs}

Un modèle adéquat pour une théorie des champs en $\infty$-groupoides est la théorie des préfaisceaux simpliciaux (voir [30, 28, 29, 2, 13]). Ainsi, le mot champ signifiera pour nous un objet de la catégorie homotopique des préfaisceaux simpliciaux. Pour un anneau $k$, nous considérerons la catégorie des schémas affines sur Speck, munie de la topologie fidèlement plate et quasi-compacte. La catégorie homotopique des préfaisceaux simpliciaux sur ce site sera notre catégorie des champs sur Speck.

D'après les travaux fondamentaux [30, 28] la théorie homotopique des champs admet une structure de modèles. Une conséquence importante, et non triviale, de l'existence de cette structure de modèles est l'existence de constructions standards tel que les limites et colimites homotopiques. De façon plus précise la catégorie de modèles des préfaisceaux simpliciaux est un topos de modèles, au sens de [60]. Ceci implique en particulier des propriétés d'exactitudes additionelles de la théorie des champs, comme par exemple l'existence de Hom internes. Il est bon de garder à l'esprit que la théorie des champs fonctionne de façon tout à fait similaire à celle des faisceaux. Une présentation de quelques constructions et propriétés standards de la théorie des champs fait l'objet du chapitre $\S 1$.

\section{Champs affines}

La notion de champs affines sur un anneau $k$ est une version dérivée de la notion de schémas affines, et généralise celle-ci dans un cadre homotopique. Pour définir cette notion nous utiliserons une structure de catégorie de modèles sur la catégorie des $k$-algèbres co-simpliciales. Pour $A$ une telle algèbre co-simpliciale, nous considérerons le schéma affine simplicial $\operatorname{Spec} A$, ainsi que le préfaisceau simplicial sur le site des $k$-schémas affines qu'il représente. Nous définissons ainsi un foncteur

$$
\text { Spec }:\left(k-A l g^{\Delta}\right)^{o p} \longrightarrow S \operatorname{Pr}(k),
$$

de la catégorie des $k$-algèbres co-simpliciales, vers la catégorie des préfaisceaux simpliciaux sur le site des $k$-schémas affines pour la topologie fidèlement plate et 
quasi-compacte. Notre première observation, qui est au centre du présent travail est la proposition suivante.

Proposition 0.0.1. (Voir Prop. 2.2.2 et Cor. 2.2.3) Le foncteur Spec est un foncteur de Quillen à droite. De plus, le foncteur dérivé

$$
\mathbb{R} \text { Spec : } H o\left(k-A l g^{\Delta}\right)^{o p} \longrightarrow H o(S P r(k))
$$

est pleinement fidèle.

Avant d'aller plus loin dans la définition des champs affines il me semble important de faire deux remarques.

- Lorsque la catégorie des schémas affines sur $k$ est munie de la topologie triviale, la proposition précédente est plus ou moins une trivialité. En contre partie, lorsque l'on travaille avec la topologie fidèlement plate, la preuve du fait que Spec est un foncteur de Quillen à droite utilise un résultat récent de D. Dugger, S. Hollander et D. Isaksen qui caractérise les préfaisceaux simpliciaux fibrants (voir [13]). Il est important de noter ici que l'utilisation de la topologie fidèlement plate est cruciale pour les résultats qui vont suivre, qui sont de toute évidence faux pour la topologie triviale. Par exemple, une des propriétés fondamentales de cette topologie que nous utiliserons est le fait que la sous-catégorie pleine de la catégorie des faisceaux en groupes, formée des faisceaux représentables par des schémas en groupes affines, est stable par conoyaux et limites projectives (du moins lorsque $k$ est un corps). Cette propriété sera très importante pour les théorèmes fondamentaux 0.0.2 et 0.0 .4 .

- La proposition 0.0.1 implique donc que la théorie homotopique des $k$-algèbres co-simpliciales se plongent dans celle des champs sur Speck pour la topologie fidèlement plate. Le foncteur $\mathbb{R} S p e c$ permet ainsi de géométriser les objets algébriques que sont les $k$-algèbres co-simpliciales. De plus, lorsque $k$ est de caractéristique nulle, les théories homotopiques des $k$-algèbres co-simpliciales et des $k$-algèbres différentielles graduées commutatives et en degrés positifs sont équivalentes (voir [21]). Ainsi, nous obtenons aussi un plongement de la catégorie homtopique des $k$-algèbres différentielles graduées commutatives (et en degrés positifs) dans la catégorie des champs sur Speck.

Nous définissons la catégorie des champs affines sur $k$ commme la souscatégorie pleine des champs sur Spec $k$ image essentielle du foncteur $\mathbb{R} S p e c$. Ainsi, en caractéristique nulle, la catégorie des champs affines sur $k$ est anti-équivalente à la catégorie homotopique des $k$-algèbres différentielles graduées commutatives et en degrés positifs. Il se trouve que la catégorie des champs affines ainsi définie possède plusieurs descriptions équivalentes. Nous commencerons par montrer que les champs affines sont les objets locaux pour la théorie cohomologique représentée par $\mathbb{G}_{a}$, et de taille raisonnable (voir Thm. 2.2.9). On diposera ainsi d'une interprétation de nature cohomologique de la notion de champs affines. Nous montrerons aussi que la catégorie des champs affines est aussi la plus petite souscatégorie pleine de la catégorie des champs sur Speck contenant les champs de la 
forme $K\left(\mathbb{G}_{a}, n\right)$ et qui est stable par limites homotopiques (voir la remarque qui suit le théorème 2.2.9). Ceci montre en particulier que la notion de champs affines est intrinsèque à la théorie des champs sur Spec $k$. Enfin, lorsque $k$ est un corps, la notion de champs affines se simplifie quelque peu, et nous donnerons alors la caractérisation suivante des champs affines, pointés et connexes.

Théorème 0.0.2. (Voir Thm. 2.4.1 et Thm. 2.4.5) Les champs affines, pointés et connexes sur un corps $k$ sont exactement les préfaisceaux simpliciaux pointés et connexes $F$, tels que pour tout $i>0$ le faisceau $\pi_{i}(F, *)$ soit représentable par un schéma en groupes affine et unipotent.

En corollaire de ce théorème on obtient qu'il existe un équivalence entre la théorie homotopique des $k$-algèbres co-simpliciales (ou encore $k$-algèbres différentielles graduées si $k$ est de caractéristique nulle) augmentées et connexes et celle des champs pointés vérifiant les conditions du théorème 0.0.2. Cette inteprétation géométrique des $k$-algèbres co-simpliciales est à ma connaissance un résultat nouveau, tout particulièrement dans le cas où $k$ est de caractéristique positive. C'est aussi un théorème non-trivial car il permet par exemple de retrouver (et ce de façon purement formelle) les théorèmes fondamentaux de l'homotopie rationnelle et $p$-adique (voir Thm. 2.5.1 et Cor. 2.5.3).

\section{Affination des types d'homotopie}

Les champs affines forment une catégorie de champs qui donne une solution problème de la schématisation de A. Grothendieck tel que je l'ai personnellement compris (voir l'appendice A). Dans ce cadre il s'interprétera de la façon suivante.

Si $k$ est un anneau, on peut considérer le foncteur dérivé des sections globales $\mathbb{R} \Gamma$, de la catégorie des champs affines sur $k$ vers la catégorie homotopique des ensembles simpliciaux. Notre premier résultat d'existence est le suivant, et est une conséquence immédiate de la proposition 0.0.1.

Corollaire 0.0.3. (voir Cor. 2.3.3) Le foncteur $\mathbb{R} \Gamma$ restreint aux champs affines possède un adjoint à gauche $X \mapsto(X \otimes k)^{\text {uni }}$. De plus, si $k^{X}$ est la $k$-algèbre co-simpliciale de cohomologie de $X$ à valeurs dans $k$ on a

$$
(X \otimes k)^{u n i} \simeq \mathbb{R} \operatorname{Spec}\left(k^{X}\right) .
$$

Le champ affine $(X \otimes k)^{\text {uni }}$ est appelé une affination de $X$ sur $k$.

Il est important de remarquer que le fait que les champs $K\left(\mathbb{G}_{a}, n\right)$ soient des champs affines implique qu'il existe une propriété de conservation de la cohomologie $H^{*}(X, k) \simeq H^{*}\left((X \otimes k)^{u n i}, \mathbb{G}_{a}\right)$ (qui est une des propriétés chères à A. Grothendieck dans [18]). Ainsi, comme les champs affines sont des objets locaux pour la théorie cohomologique représentés par $\mathbb{G}_{a}$, le morphisme d'adjonction $X \longrightarrow(X \otimes k)^{u n i}$ peut aussi s'interpréter comme un morphisme de localisation (dans le sens que A.K. Bousfield a donné à ce terme dans [3]).

En utilisant le théorème 0.0 .2 , il est possible de décrire le champ $(X \otimes k)^{u n i}$, du moins lorsque $X$ est nilpotent de type fini et $k$ est un corps. Dans ce cas, nous 
montrerons que $(X \otimes \mathbb{Q})^{u n i}$ est un modèle pour le type d'homotopie rationnel de $X$, et $\left(X \otimes \overline{\mathbb{F}}_{p}\right)^{u n i}$ un modèle pour son type d'homotopie $p$-adique (voir Thm. 2.5.1 et Cor. 2.5.3). Ces résultats sont des conséquences formelle de la propriété universelle satisfaite $\operatorname{par}(X \otimes k)^{u n i}$ et du théorème 0.0.2. La notion de champ affine permet ainsi de construire des modèles géométrico-algébriques aux types d'homotopie.

Enfin, signalons que lorsque $X$ est un ensemble simplicial fini, le foncteur $X \mapsto(X \otimes k)^{u n i}$ est compatible aux changements d'anneaux. De ce fait, le champ affine $(X \otimes \mathbb{Z})^{u n i}$ permet d'unifier les types d'homotopie rationnel et $p$-adique de $X$. Nous donnerons une description conjecturale de ce champ lorsque $X$ est 1connexe et de type fini au paragraphe $\S 2.3$ (voir Conj. 2.3.6).

\section{$\infty$-Gerbes affines et types d'homotopie schématiques}

De par leur définition, les types d'homotopie représentés par des champs affines ont une forte tendence à être nilpotents. Ceci est un fait très bien connu en théorie de l'homotopie rationnelle ou $p$-adique, où les groupes fondamentaux que l'on obtient à partir des modèles algébriques sont les complétions de Mal'cev (relativement au corps de base) des groupes fondamentaux usuels. De même, pour un espace $X$ qui est non-nilpotent, le champ $(X \otimes k)^{u n i}$ ne verra pas la partie réductive du type d'homotopie de $X$ (e.g. le schéma en groupes $\pi_{1}\left((X \otimes k)^{u n i}, x\right)$ est un schéma en groupes affine pro-unipotent). On est donc naturellement amenés à introduire une notion plus générale que celle de champs affines qui soit moins restrictive au niveau des groupes fondamentaux. C'est pour cette notion plus générale que nous réserverons l'expression types d'homotopie schématiques.

Tout d'abord nous introduirons une notion d' $\infty$-gerbe affine qui généralise la notion de gerbe affine utilisée dans le formalisme Tannakien (voir [43, 10]), et de façon à ce que les $\infty$-gerbes affines soient aux gerbes affines ce que les champs affines sont aux schémas affines. En clair, nous définirons une $\infty$-gerbe affine (pointée) sur un corps $k$ comme étant un champ pointé et connexe $F$ sur Speck, dont le champ des lacets $\Omega_{*} F$ est un champ affine sur $k$ (voir Def. 3.1.2). Les types d'homotopie schématiques pointés sur $k$ seront alors les $\infty$-gerbes affines pointées possédant une certaine propriété de localité cohomologique (voir Def. 3.1.2). Nous conjecturons cependant que cette propriété est toujours satisfaite, et que toute $\infty$-gerbe affine pointée est donc un type d'homotopie schématique (voir 3.2.10). Quoiqu'il en soit, la catégorie des types d'homotopie schématiques pointés ainsi définie est une sous-catégorie pleine de la catégorie homotopique des préfaisceaux simpliciaux pointés sur le site des schémas affines sur le corps $k$. Les $\infty$-gerbes affines et les types d'homotopie schématiques sur un anneau de base plus général ne seront pas définis dans ce travail.

Le premier résultat que nous démontrerons est le critère de reconnaissance suivant, qui permet de donner de nombreux exemples intéressants de types d'homotopie schématiques pointés. 
Théorème 0.0.4. (Voir Thm. 3.2.4 et Thm. 3.2.9) Les types d'homotopie schématiques sont exactement les préfaisceaux simpliciaux pointés et connexes tels que pour tout $i>0$, le faisceau $\pi_{i}(F, *)$ soit représentable par un schéma en groupes affine, qui est de plus unipotent pour $i>1$.

De plus, si $F$ est une $\infty$-gerbe affine pointée, alors pour tout $i>1$ le faisceau $\pi_{i}(F, *)$ est représentable par un schéma en groupes affine et unipotent. Le faisceau $\pi_{1}(F, *)$ est un sous-faisceau d'un faisceau représentable par un schéma en groupes affine.

Le théorème précédent répond presque à la conjecture 3.2.10. Pour donner une preuve complète à cette conjecture il resterait à montrer que pour toute $\infty$ gerbe affine pointée $F$, le faisceau $\pi_{1}(F, *)$ soit représentable par un schéma affine. On peut en réalité montrer que le faisceau $\pi_{1}(F, *)$ possède un espace de modules grossier affine $\widetilde{\pi_{1}(F, *)}$, et que le morphisme naturel $\pi_{1}(F, *) \longrightarrow \widetilde{\pi_{1}(F, *)}$ est un monomorphisme.

Les types d'homotopie schématiques forment une catégorie de champs donnant une solution du problème de la schématisation (au sens de l'appendice A). De façon plus précise, pour un corps $k$, on peut considérer le foncteur dérivé des sections globales $\mathbb{R} \Gamma$, de la catégorie des types d'homotopie schématiques pointés sur $k$ vers la catégorie homotopique des ensembles simpliciaux pointés. Notre second théorème d'existence est le suivant.

Théorème 0.0.5. (voir Thm. 3.3.4) Le foncteur $\mathbb{R} \Gamma$ restreint à la catégorie des types d'homotopie schématiques pointés possède un adjoint à gauche $X \mapsto(X \otimes k)^{\text {sch }}$. Le champ $(X \otimes k)^{\text {sch }}$ sera appelé une schématisation de $X$ sur $k$.

On déduit immédiatement de la propriété universelle des schématisations que le schéma en groupes affine $\pi_{1}\left((X \otimes k)^{s c h}, x\right)$ est le complété affine du groupe discret $\pi_{1}(X, x)$. Il s'agit donc du groupe fondamental algébrique de l'espace $X$ (décrit par exemple dans [9] et [43, VI $\S 1]$ ). On entrevoit ici la différence fondamentale entre les foncteurs d'affination et de schématisation. Il existe en effet toujours un morphisme naturel $(X \otimes k)^{s c h} \longrightarrow(X \otimes k)^{u n i}$, qui au niveau des groupes fondamentaux induit la projection de $\pi_{1}\left((X \otimes k)^{s c h}, x\right)$ sur son quotient unipotent maximal. Ce morphisme au niveau des champs peut aussi être raisonnablement pensé comme un quotient unipotent maximal.

Tout comme pour le cas des affinations, le morphisme d'adjonction $X \longrightarrow$ $(X \otimes k)^{s c h}$ possède une certaine propriété de préservation de la cohomologie. Plus précisèment, le groupe $\pi_{1}(X, x)$ et le faisceau en groupes $\pi_{1}\left((X \otimes k)^{s c h}, x\right)$ possèdent les mêmes représentations linéaires. De plus, pour $V$ une telle représentation linéaire on a $H^{*}(X, V) \simeq H^{*}\left((X \otimes k)^{s c h}, V \otimes \mathbb{G}_{a}\right)$. Ces deux conditions peuvent aussi se combiner en une notion de $P$-équivalence (voir Def. 3.1.1), ce qui permet d'affirmer que le morphisme $X \longrightarrow(X \otimes k)^{s c h}$ est aussi un morphisme de localisation au sens de A.K. Bousfield. 
Enfin, tout comme pour le cas du foncteur d'affination, le foncteur $X \mapsto$ $(X \otimes k)^{s c h}$ permet de modeler une certaine partie de la théorie de l'homotopie (une partie que l'on pourra appeler schématique). Cependant, même pour des types d'homotopie de type fini $X$, il n'est pas raisonnable de penser pouvoir décrire explicitement le champ $(X \otimes k)^{s c h}$. Ainsi, ce que modéle exactement les types d'homotopie schématiques reste un peu mystérieux. Quoiqu'il en soit, des exemples simples montrent que le champ $(X \otimes k)^{s c h}$ est généralement beaucoup plus gros que le champ $(X \otimes k)^{\text {uni }}$ (voir $\S 3.4$ ). Enfin, lorsque $X$ est simplement connexe on a $(X \otimes k)^{s c h} \simeq(X \otimes k)^{u n i}$. L'objet $(X \otimes k)^{s c h}$ nous semble donc un substitut bien adapté à la théorie de l'homotopie rationnelle et $p$-adique pour les types d'homotopie non-simplement connexes. A titre d'exemple d'application, la construction $X \mapsto(X \otimes \mathbb{C})^{s c h}$ sera utilisée dans un travail ultérieur pour étudier les types d'homotopie des variétés complexes lisses et projectives à groupes fondamentaux arbitraires. L'étude par la théorie de Hodge de ce nouvel invariant d'homotopie permettra alors d'obtenir de nouvelles restrictions sur les types d'homotopie des variétés projectives, qui semblent hors d'atteinte par une approche utilisant la théorie de l'homotopie rationnelle (voir [31] ainsi que §3.5.1).

Signalons aussi que les algèbres co-simpliciales sont des modèles algébriques pour les champs affines. De même, il existe une notion de $H_{\infty}$-algèbres de Hopf qui permet de donner des modèles algébriques des types d'homotopie schématiques (voir Def. 3.1.4). L'étude de ces modèles algébriques ne sera pas le but de ce travail, mais nous espérons pouvoir revenir sur le sujet par la suite (voir [32]). A titre indicatif, on peut résumer le double apport de cet article par le diagramme symbolique suivant.

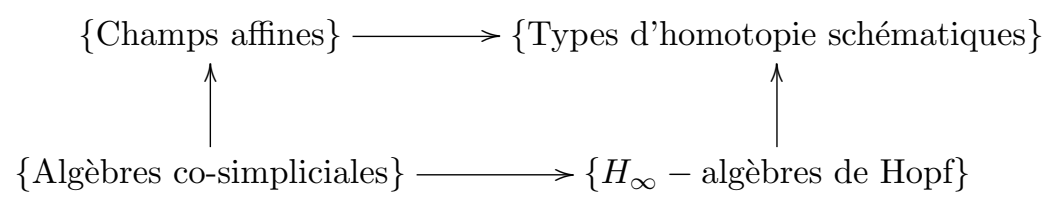

Dans ce diagramme, les flèches verticales sont les procédés de géométrisation induit par le foncteur $\mathbb{R} S p e c$ (i.e. le passage de l'algèbre à la géométrie), et les fléches horizontales symbolisent le passage aux objets en groupes.

\section{Types d'homotopie des variétés algébriques}

La théorie générale des types d'homotopie schématiques et du foncteur de schématisation possède plusieurs applications dans le cadre de la géométrie algébrique. Une première application est l'étude des types d'homotopie des variétés algébriques complexes (voir §3.5.1). En effet, pour une variété complexe lisse et projective $X$ et $x \in X(\mathbb{C})$, on peut considérer son espace topologique sousjacent des points complexes (muni de la topologie analytique) $X^{t o p}$, ainsi que sa schématisation $\left(X^{\text {top }} \otimes \mathbb{C}\right)^{\text {sch }}$. Dans le travail [31] on construit une décomposition de Hodge sur le champ $\left(X^{\text {top }} \otimes \mathbb{C}\right)^{s c h}$, qui englobe les différentes structures de 
Hodge connues sur la cohomologie, les groupes d'homotopie et le groupe fondamental. L'existence de cette structure additionelle sur le champ $\left(X^{\text {top }} \otimes \mathbb{C}\right)^{\text {sch }}$ possède d'intéressantes conséquences sur le type d'homotopie de la variété $X$ et impose de sérieuses restrictions. On peut ainsi construire des nouveaux exemples de types d'homotopie qui ne sont pas réalisables par des variétés projectives, et dont l'obstruction se trouve dans des invariants d'homotopie supérieurs (précisemment l'action du groupe fondamental sur les groupes d'homotopie).

De façon plus générale pour une variété algébrique $X$ sur un corps de base $k$ on peut utiliser la notion de types d'homotopie schématiques afin de construire des types d'homotopie associés aux théories cohomologiques usuelles (Betti, de Rham, $l$-adique, crystalline, $\ldots$, voir $§ 3.5 .2,3.5 .3)$. Toutes ces constructions montrent que la notion de type d'homotopie schématique est adéquate pour l'étude homotopique des variétés algébriques, tout comme il était déjà bien connu que le bon cadre pour une théorie des groupes fondamentaux de variétés algébriques est en réalité celui des schémas en groupes affines et non celui des groupes discrets (voir par exemple $[9,43])$. Cependant, ces constructions ne seront pas décrites en détail et nous nous contenterons de donner les propriétés fondamentales (et parfois caractéristiques) de ces types d'homotopie.

\section{Champs $\infty$-géométriques}

Une autre application de la théorie des champs affines est la notion de champ $\infty$-géométrique (voir §4). Par définition un champ algébrique (par exemple au sens d'Artin) est obtenu en recollant des schémas affines à l'aide de l'action d'un groupoide lisse. En remplaçant formellement la notion de schéma affine par celle plus générale de champ affine on donne une définition de champ $\infty$-géométrique. Il se trouve que les types d'homotopie schématiques fournissent les premiers exemples de champs $\infty$-géométriques. Un deuxième exemple fondamental est le champ des structures multiplicatives sur un complex parfait $V$, généralisant le champ des structures d'algèbres sur un espace vectoriel donné au cas des algèbres différentielles graduées (voir §4.2.1). On peut aussi donner des exemples plus sophistiqués de champs $\infty$-géométriques, comme le champ des filtrations sur une algèbre différentielle graduée commutative, qui pourra être utilisé pour construire un domaine des périodes non-abéliennes ainsi qu'une application de périodes correspondantes qui controlera la variation de la filtration de Hodge sur le type d'homotopie rationnel d'une famille de variétés lisses et projectives (voir §4.2.2).

\section{Relations avec d'autres travaux}

La notion de champs affine me semble nouvelle. Il en existe cependant des avatars ou versions voisines dans les articles [53, §6] et [20]. En caractéristique nulle et pour le cas des types d'homotopie 1-connexes et de type fini le théorème 0.0 .2 apparait dans $[53, \S 6]$.

Il y a aussi les quelques six cents pages de [18], dont il est extrèmement difficile de faire une comparaison avec le point de vue adopté ici. Par exemple, la 
notion de champs affines me semble assez proche de celle de complexes of unipotent bundles discutés dans [18, p. 446]. Cependant, l'objet que A. Grothendieck appelle schématisation d'un espace $X$ semble plus proche de notre affination $(X \otimes k)^{u n i}$ que de notre schématisation $(X \otimes k)^{s c h}$. A vrai dire la question de l'existence du champ $(X \otimes k)^{s c h}$, bien que fortement inspirée des considérations sur la théorie de l'homotopie que l'on trouve dans les lettres de A. Grothendieck à L. Breen, ne semble pas avoir été considérée dans [18]. Cependant, la motivation principale de ce travail est née d'un désir de comprendre certains passages de [18], et de ce fait ce manuscrit a eu une influence déterminante sur les définitions et les énoncés qui apparaissent dans cet article. Ce travail s'insère donc naturellement dans le vaste programme proposé par C. Simpson et intitulé la poursuite de la poursuite des champs (ou encore comme l'a suggéré D. Husemöller lors d'une conversation sur le sujet la 2-poursuite des champs).

Il existe aussi d'autres approches au problème de la schématisation des types d'homotopie tel qu'il est abordé dans [18]. La première, qui est exposée dans [6], donne une construction de la sphère schématique stable en caractéristique positive. Il n'est pas tout à fait clair que cette construction soit comparable à celle de cet article, même si cette sphère schématique stable ressemble de très près à ce que l'on pourrait appeler dans notre langage un spectre affine (version stable des champs affines). Une seconde approche a été introduite dans [51]. Ici aussi la comparaison avec notre construction n'est pas immédiate, bien que la théorie des champs présentables et très présentables soit intimement liée à celle des types d'homotopie schématiques (voir Thm. 3.2.4). Enfin, dans [58] nous avons construit un foncteur de schématisation à l'aide d'une notion de catégorie simpliciale Tannakienne, qui est conjecturé être isomorphe à celui défini dans ce travail (voir aussi [59]). Signalons au passage que les constructions et résultats du présent travail ont étés pour la plupart devinés à l'aide de ce formalisme Tannakien.

Comme nous l'avons fait remarquer le foncteur de schématisation que nous construisons peut être considéré comme un substitut des théories de l'homotopie rationnelle et $p$-adique pour des espaces non-nilpotents. Il existe aussi une autre approche qui consiste à utiliser une version équivariante de l'homotopie rationnelle et $p$-adique, et à appliquer celle-ci aux revêtements universels (voir [7, 17]). Il me semble un problème intéressant de savoir comment ces deux points de vue sont reliés.

Enfin, la notion de champs $\infty$-géométriques présenté dans le paragraphe $\S 4$ a été inspirée par [53]. Elle est aussi tout à fait analogue a la notion de $D$-champs géométriques de [62] (voir aussi [61]). Pour tout dire la théorie des champs affines de cet article a fortement inspiré le développement de la géométrie algébrique homotopique de [60,61].

\section{Remerciements}

Je voudrais tout d'abord remercier C. Simpson, qui par l'intermédiaire d'articles, de discussions et de correspondances a beaucoup influencé ce travail. Je 
remercie aussi particulièrement H. Baues, qui m'a suggéré d'utiliser une version dérivée du foncteur de complétion affine des groupes discrets afin de construire le foncteur de schématisation. Le lecteur remarquera que ceci est une étape fondamentale dans la preuve du théorème 3.3.4.

Pour de nombreuses discussions, remarques et correspondances sur le sujet je remercie aussi les personnes suivantes: L. Breen, A. Hirschowitz, L. Katzarkov, M. Olsson, T. Pantev, J. Sauloy, M. Spitzweck, D. Stanley, J. Tapia et G. Vezzosi.

La majeure partie de ce travail a été effectuée à l'institut Max Planck de Bonn durant l'année 1999 - 2000, que je tiens à remercier pour son hospitalité et ses conditions de travail exceptionnelles. 
Notations et conventions: Pour un univers $\mathbb{U}$ nous appellerons $\mathbb{U}$-ensemble (resp. U-ensemble simplicial, resp. U-groupe, resp. ...) un ensemble (resp. un ensemble simplicial, resp. un groupe, resp. ... ) qui est un élément de U. La catégorie des $\mathbb{U}$-ensembles (resp. U-ensembles simpliciaux, resp. U-groupes, resp ...) sera alors notée $\mathbb{U}-E n s$ (resp. $\mathbb{U}-S E n s$, resp. $\mathbb{U}-G p$, resp. ... ). Nous ferons une exception avec l'expression U-catégorie, que nous réservons à la notion usuelle (voir [48, IDef.1.2]), désignant une catégorie $C$ telle que étant donné deux objets $X$ et $Y$, l'ensemble des morphismes de $X$ vers $Y$ dans $C$ soit un $\mathbb{U}$-ensemble. Pour désigner une catégorie appartenant à $\mathbb{U}$ nous parlerons de catégorie $\mathbb{U}$-petite.

Nous parlerons de $\mathbb{U}$-limites et $\mathbb{U}$-colimites (resp. $\mathbb{V}$-limites et $\mathbb{V}$-colimites) pour désigner des limites et colimites dont les catégories d'indices appartiennent à $\mathbb{U}($ resp. à $\mathbb{V})$. De même, nous parlerons de $\mathbb{U}$-limites et $\mathbb{U}$-colimites homotopiques (resp. $\mathbb{V}$-limites et $\mathbb{V}$-colimites homotopiques) à valeurs dans une catégorie de modèles (voir $[22, \S 20]$ ).

Pour la suite nous fixerons $\mathbb{U}$ un univers contenant l'ensenbles des nombres naturels, et $\mathbb{V}$ un univers avec $\mathbb{U} \in \mathbb{V}$. Comme il en est l'usage, nous noterons $\Delta$ la catégorie simpliciale standard. C'est la catégorie U-petite dont les objets sont les nombres naturels $[n]$, et dont les morphismes de $[m]$ vers $[n]$ sont les applications croissantes de $\{0, \ldots, m\}$ vers $\{0, \ldots, n\}$. Pour tout $\mathbb{V}$-catégorie $C$, nous noterons $S C$ la $\mathbb{V}$-catégorie des objets simpliciaux dans $C$ (i.e. la $\mathbb{V}$-catégorie des foncteurs de $\Delta^{o}$ vers $C$ ), et pour $F \in S C$ nous noterons $F_{n}:=F([n])$. Nous identifierons systématiquement $C$ à la sous-catégorie pleine de $C^{\Delta^{\circ}}$ formée des foncteurs constants $F: \Delta^{o} \longrightarrow C$.

Si $C$ est une catégorie $\mathbb{V}$-petite, et $X$ un objet de $C$, nous noterons $h_{X}$ le $\mathbb{V}$-préfaisceau simplicial sur $C$ qu'il représente. C'est donc le foncteur défini par $h_{X}(Y):=\operatorname{Hom}(Y, X)$, où l'ensemble $\operatorname{Hom}(Y, X)$ est vu comme un ensemble simplicial constant. De même, si $X$ est un objet simplicial de $C$, nous noterons $h_{X}$ le $\mathbb{V}$-préfaisceau simplicial dont le préfaisceau des simplexes de dimension $n$ est défini par la formule $\left(h_{X}\right)_{n}(Y):=\operatorname{Hom}\left(Y, X_{n}\right)$.

Nous utiliserons les définitions de catégories de modèles fermées énoncées dans [26]. Elles seront toujours $\mathbb{V}$-petites. On supposera implicitement lorsqu'elles seront engendrées par cofibrations (voir [26, §2.1]) que les ensembles générateurs des cofibrations et cofibrations triviales seront des $\mathbb{V}$-ensembles (de même, les ordinaux apparaissant dans les colimites transfinies seront des éléments de $\mathbb{V}$ ). Rappelons que la catégorie $\mathbb{V}-S E n s$ est une catégorie de modèles fermée, monoïdale symétrique pour le produit direct, et engendrée par cofibrations pour la convention ci-dessus (voir $[26, \S 3]$ ). Les Hom internes de $\mathbb{V}-S E n s$ seront notés $\underline{\text { Hom }}$, et ces Hom dérivés $\mathbb{R} \underline{H o m}$ (voir $[26, \S 4]$ ). Une catégorie de modèles fermée simpliciale sera alors une catégorie ( $\mathbb{V}$-petite) de modèles fermée, qui est un module sur $\mathbb{V}-S E n s$ (au sens de $[26,4.2 .28]$ ). Enfin, si $M$ est une catégorie de modèles fermée $H o(M)$ désignera sa catéorie homotopique. C'est une catégorie $\mathbb{V}$-petite 
qui est naturellement équivalente à une U-catégorie. Les ensembles de morphismes dans $H o(M)$ seront notés $[-,-]_{M}$.

Enfin, pour une catégorie de modèles fermée $M$ nous noterons $M_{*}$ la catégorie de modèles de ses objets pointés. Lorsque de plus $M$ est une catégorie de modèles simpliciale, il en est de même de $M_{*}$. Ses Hom simpliciaux seront alors notés $\underline{\mathrm{Hom}}_{*}($ voir $[26,4.2 .19])$. 


\section{Rappels sur les champs}

Dans ce premier chapitre nous avons rassemblé un certains nombres de définitions et de résultats standards de la théorie des préfaisceaux simpliciaux sur un site de Grothendieck. Les idées essentielles de la théorie ont été développées par A. Joyal dans une lettre à A. Grothendieck (voir [30]), où l'existence d'une structure de catégorie de modèles sur la catégorie des faisceaux simpliciaux est démontrée. Nous utiliserons cependant une structure un peu différente, où la classe des cofibrations est strictement plus petite que celle des monomorphismes, et où les objets fibrants sont faciles à caractériser (voir 1.1.2). Basée sur des idées remontant à D. Quillen, A.K. Bousfield et D.M. Kan, cette structure à été considérée pour la première fois dans [23, 5], et a été reprise en détail dans [2]. Pour ce qui est des preuves et des détails techniques nous renvoyons le lecteur à $[28,23,15,23,2]$. Signalons aussi le travail en cours [13], où il est probable que le point de vue de la localisation de Bousfield décrit dans $[23,6]$ soit repris en détail.

Nous commencerons par rappeler la définition de la structure de catégorie de modèles fermée sur la catégorie des préfaisceaux simpliciaux, ainsi que ces principales propriétés. L'existence de cette structure de catégorie de modèles nous sera utile tout au long de ce travail. Dans les paragraphes suivants nous rappellerons comment elle permet de définir la cohomologie d'un préfaisceau simplicial à valeurs dans un système local. Nous donnerons aussi une brève démonstration d'un théorème de comparaison avec la cohomologie définie par foncteurs dérivés, ainsi qu'un critère pour s'assurer qu'un préfaisceau simplicial possède une décomposition de Postnikov convenable. Nous terminerons ce chapitre par un aperçu de la théorie du délaçage dans le cadre des préfaisceaux simpliciaux, et un bref rappel sur les schémas en groupes affines sur un corps.

Pour tout ce chapitre $C$ désignera une catégorie $\mathbb{V}$-petite, et $\operatorname{SPr}(C)$ la catégorie des préfaisceaux en $\mathbb{V}$-ensembles simpliciaux sur $C$. La catégorie $\operatorname{Pr}(C)$ est donc une $\mathbb{V}$-catégorie. Nous supposerons que $C$ possède des produits fibrés, et qu'elle est munie d'une topologie de Grothendieck (au sens de $[47, I V]$ ). Pour fixer les idées, notons que par la suite $C$ sera la catégorie des U-schémas affines sur Speck, pour $k$ un anneau commutatif, et la topologie sera engendrée par les morphismes fidèlement plats et quasi-compacts (i.e. la topologie $f p q c$ de $[47, I V .6 .3]$ ).

\subsection{Rappel des définitions}

On commence par munir la catégorie $S \operatorname{Pr}(C)$ d'une structure de catégorie de modèles que nous appellerons le structure forte (dans la littérature on trouve aussi l'expression de structure projective niveau par niveau), et qui a étée introduite pour la première fois dans [5]. Il s'agit du cas où l'on considère la topologie discrète, et donc où l'on voit $C$ comme une simple catégorie d'indices. On appellera alors fibration forte (resp. équivalence forte) tout morphisme de $\operatorname{PPr}(C), f: F \longrightarrow F^{\prime}$, tel que pour tout objet $X \in C$ le morphisme $f_{X}: F(X) \longrightarrow F\left(X^{\prime}\right)$ soit une 
fibration (resp. une équivalence) dans $\mathbb{V}-S E n s$. Pour ces définitions, $C$ étant $\mathbb{V}$ petite, la catégorie $S \operatorname{Pr}(C)$ est une catégorie de modèles fermée simpliciale (voir [15, Ex.II.6.9]). Les Hom simpliciaux de cette dernière seront notés $\underline{\text { Hom }}$. En clair, ceci signifie que pour $X \in \mathbb{V}-S E n s$ et $F, F^{\prime} \in S \operatorname{Pr}(C)$ on peut définir fonctoriellement dans $S P r(C)$ des objets $X \otimes F, \underline{\operatorname{Hom}}\left(F, F^{\prime}\right)$ et $\left(F^{\prime}\right)^{X}$ satisfaisant à la formule d'adjonction

$$
\underline{\operatorname{Hom}}\left(X, \underline{\operatorname{Hom}}\left(F, F^{\prime}\right)\right) \simeq \underline{\operatorname{Hom}}\left(X \otimes F, F^{\prime}\right) \simeq \underline{\operatorname{Hom}}\left(F,\left(F^{\prime}\right)^{X}\right) .
$$

Ces Hom simpliciaux étant compatibles avec la structure de catégorie de modèles on peut définir des Hom simpliciaux dérivés (voir [26, §4.3])

$$
\mathbb{R}_{f} \underline{\operatorname{Hom}}\left(F, F^{\prime}\right):=\underline{\operatorname{Hom}}\left(Q F, R F^{\prime}\right) \in H o(\mathbb{V}-S E n s),
$$

où $Q F \longrightarrow F$ est un remplacement cofibrant, et $F^{\prime} \longrightarrow R F^{\prime}$ un remplacement fibrant. Noter l'indice $f$ dans la notation $\mathbb{R}_{f}$, qui fait référence à la structure forte. Ces objets sont bien définis et fonctoriels comme objets de la catégorie homotopique $H o(\mathbb{V}-S E n s)$. De plus, la formule d'adjonction précédente induit une formule d'adjonction dérivée

$$
\mathbb{R}_{f} \underline{\operatorname{Hom}}\left(X, \mathbb{R}_{f} \underline{\operatorname{Hom}}\left(F, F^{\prime}\right)\right) \simeq \mathbb{R}_{f} \underline{\operatorname{Hom}}\left(X \otimes F, F^{\prime}\right) \simeq \mathbb{R}_{f} \underline{\operatorname{Hom}}\left(F,\left(R F^{\prime}\right)^{X}\right),
$$

où $F^{\prime} \longrightarrow R F^{\prime}$ est un remplacement fibrant.

Pour $X \in C$, on dispose du foncteur d'évaluation en $X$

$$
\begin{aligned}
& j_{X}: \quad S P r(C) \longrightarrow \mathbb{V}-\text { SEns } \\
& F \quad \mapsto \quad F(X)
\end{aligned}
$$

Ce foncteur possède un adjoint à gauche noté $j_{X}^{*}$, et l'adjonction $\left(j_{X}^{*}, j_{X}\right)$ est une adjonction de Quillen. En particulier, pour tout $X \in C, j_{X}^{*}(*)$ est un objet cofibrant de $S \operatorname{Pr}(C)$. Or, il est clair que $j_{X}^{*}(*)$ est isomorphe à $h_{X}$. Ainsi, pour tout objet du site $X \in C$, le préfaisceau simplicial qu'il représente $h_{X}$ est un objet cofibrant. Dans le cas où $X=e$ est un objet final, nous noterons comme il en est l'usage

$$
\begin{array}{ccc}
\Gamma:=j_{e}: \quad S P r(C) & \longrightarrow & \mathbb{V}-\text { SEns } \\
F & \mapsto & F(e),
\end{array}
$$

le foncteur des sections globales. Son adjoint à gauche $j_{e}^{*}$ est le foncteur qui associe à $X \in \mathbb{V}-S E n s$ le préfaisceau constant $\underline{X}$. Remarquer que lorsque $C$ ne possède pas d'objet final les préfaisceaux constants ne sont généralement pas cofibrants. De plus, le foncteur des sections globales $\Gamma(-):=\underline{H o m}(*,-)$ n'est alors plus de Quillen à droite en général.

Revenons au cas où $C$ est munie d'une topologie de Grothendieck. A un objet $F \in S \operatorname{Pr}(C)$ on associe ses préfaisceaux d'homotopie de la façon suivante. Pour tout $X \in C$, et tout 0 -simplexe $s \in F(X)_{0}$, on définit le $m$-ème préfaisceau 
d'homotopie de $F$ pointé en $s$ par

$$
\begin{array}{cccc}
\pi_{m}^{p r}(F, s): & (C / X)^{o} & \longrightarrow & \mathbb{V}-G p \\
(u: Y \rightarrow X) & \mapsto & \pi_{m}\left(F(Y), u^{*}(s)\right) .
\end{array}
$$

C'est un préfaisceau en $\mathbb{V}$-groupes sur $C / X$ qui est abélien lorsque $m>1$. Le préfaisceau des composantes connexes de $F$ est défini par

$$
\begin{aligned}
\pi_{0}^{p r}(F): & C^{o} \\
X & \mapsto \mathbb{V}-\text { Ens } \\
X & \pi_{0}(F(X)) .
\end{aligned}
$$

On définit alors les faisceaux d'homotopie de $F$ comme étant les faisceaux associés aux préfaisceaux d'homotopie. Ils seront notés

$$
\pi_{m}(F, s):=a\left(\pi_{m}^{p r}(F, s)\right) \quad \pi_{0}(F):=a\left(\pi_{0}^{p r}(F)\right) .
$$

Définition 1.1.1. Soit $f: F \longrightarrow F^{\prime}$ un morphisme de préfaisceaux simpliciaux sur le site $C$.

- Le morphisme $f$ est une équivalence si les deux conditions suivantes sont satisfaites.

- Le morphisme induit $\pi_{0}(f): \pi_{0}(F) \longrightarrow \pi_{0}\left(F^{\prime}\right)$ est un isomorphisme de faisceaux sur $C$.

- Pour tout objet $X \in C$, tout $m>0$ et tout 0 -simplexe $s \in F(X)_{0}$, les morphismes induits

$$
\pi_{m}(f, s): \pi_{m}(F, s) \longrightarrow \pi_{m}\left(F^{\prime}, f(s)\right)
$$

sont des isomorphismes de faisceaux sur $C / X$.

- Le morphisme $f$ est une cofibration si c'est une cofibration forte.

Comme il est expliqué dans [23, Thm. 5.1], ces définitions munissent la catégorie $S \operatorname{Pr}(C)$ d'une structure de catégorie de modèles simpliciale. Remarquons au passage que toute équivalence forte est une équivalence, que toute fibration est une fibration forte, et que tout fibration triviale est une équivalence forte. De même, toute équivalence entre objets fibrants dans $S \operatorname{Pr}(C)$ est une équivalence forte.

Les Hom simpliciaux dérivés relativement à cette nouvelle structure seront notés $\mathbb{R}$ Hom . De même, lorsque $C$ possèdera un objet final $e$, le foncteur dérivé à droite des sections globales sera noté $\mathbb{R} \Gamma$. On dispose aussi des isomorphismes d'adjonctions analogues à ceux décrits précédemment

$$
\mathbb{R} \underline{\operatorname{Hom}}\left(X \times F, F^{\prime}\right) \simeq \mathbb{R} \underline{\operatorname{Hom}}\left(X, \underline{\mathbb{R}} \underline{\operatorname{Hom}}\left(F, F^{\prime}\right)\right) \simeq \mathbb{R} \underline{\operatorname{Hom}}\left(F,\left(R F^{\prime}\right)^{X}\right) .
$$

Il existe une relation entre les deux structures de catégorie de modèles sur $S \operatorname{Pr}(C)$ connue sous le nom de localisation de Bousfield à gauche (voir [22]). Nous n'insisterons pas trop sur cette notion et nous nous contenterons de retenir que le fait que $S \operatorname{Pr}(C)$ soit une localisation de Bousfield à gauche de $S \operatorname{Pr}(C)$ munie de la structure forte implique l'existence d'une jolie caractérisation des objets fibrants dans $S \operatorname{Pr}(C)$. Le lecteur remarquera qu'il s'agit d'un analogue de nature homotopique de la définition d'un faisceau. 
Lemme 1.1.2. Un objet $F \in S \operatorname{Pr}(C)$ est fibrant si et seulement si les deux assertions suivantes sont satisfaites.

1. Pour tout objet $X \in C, F(X)$ est fibrant dans $\mathbb{V}-S E n s$ (i.e. F est fortement fibrant).

2. Pour tout objet $X \in C$, et tout hyper-recouvrement $U_{*}$ de $X$ dans $C$, le morphisme naturel

$$
F(X) \longrightarrow \operatorname{Holim}_{[m] \in \Delta} F\left(U_{m}\right)
$$

est une équivalence dans $\mathbb{V}-$ SEns.

Preuve: Voir [13].

Un corollaire important de ce dernier lemme est qu'un préfaisceau simplicial de la forme $K(M, n)$, où $M$ est un faisceau flasque sur $C$, est toujours fibrant.

Remarquer que s'il l'on considère des préfaisceaux en groupoïdes plutôt que des préfaisceaux simpliciaux, alors la seconde condition du lemme précédent est exactement équivalente à la condition usuelle d'être un champ (voir [29] pour plus de détails sur les relations entre préfaisceaux simpliciaux et champs en groupoïdes). La définition suivante est alors naturelle. Notons qu'il s'agit aussi du point de vue adopté dans [23] pour développer la théorie des $n$-champs de Segal.

Définition 1.1.3. Un champ sur $C$ est un objet de la catégorie $\operatorname{Sr}(C)$ satisfaisant à la condition (2) du lemme 1.1.2. Par extension, tout objet $F$ de la catégorie homotopique $\operatorname{Ho}(S \operatorname{Pr}(C))$ sera appelé un champ.

Avec ce langage, le champ associé à un préfaisceau simplicial $F$ est simplement un de ses modèles fibrants.

\subsection{Limites homotopiques et décomposition de Postnikov}

Soit $I$ une catégorie $\mathbb{V}$-petite, et $S \operatorname{Pr}(C)^{I}$ la catégorie des foncteurs de $I$ vers $S \operatorname{Pr}(C)$. On peut munir $S \operatorname{Pr}(C)^{I}$ d'une structure de catégorie de modèles engendrée par cofibrations où les équivalences (resp. les cofibrations) sont définies niveau par niveau (i.e. $F \longrightarrow F^{\prime}$ est une équivalence si et seulement si pour tout $i \in I, F(i) \longrightarrow F^{\prime}(i)$ est une équivalence dans $\left.S \operatorname{Pr}(C)\right)$. Noter que cette structure n'est pas celle que nous avons utilisée pour définir $\operatorname{Pr}(C)$, mais est une structure de type injective analogue à celle définie dans [28, Thm. 2.3]. La catégorie de modèles ainsi obtenue est une catégorie de modèles fermée simpliciale, et ces Hom simpliciaux seront notés $\underline{H o m}_{I}$. En particulier, on dispose du foncteur des sections globales

$$
\begin{array}{ccc}
\Gamma(I,-): \quad \operatorname{SPr}(C)^{I} & \longrightarrow & \mathbb{V}-\text { SEns } \\
F & \mapsto \quad \underline{\operatorname{Hom}}_{I}(*, F)
\end{array}
$$

dont l'adjoint à gauche est le foncteur qui associe à $X \in \mathbb{V}-S E n s$ le foncteur constant de valeurs $X, \underline{X}: I \longrightarrow S \operatorname{Pr}(C)$. L'adjonction $($ 二, $\Gamma(I,-))$ est alors une 
adjonction de Quillen, et le foncteur dérivé à droite de $\Gamma(I,-)$ sera alors noté

$$
\operatorname{Holim}_{I}:=\mathbb{R} \Gamma(I,-): H o\left(\operatorname{SPr}(C)^{I}\right) \longrightarrow H o(\operatorname{SPr}(C)) .
$$

C'est le foncteur de limite homotopique le long de $I$. Il est naturellement isomorphe au foncteur Holim $_{I}$ construit de façon standard à partir de la structure simpliciale sur $S \operatorname{Pr}(C)$ (voir $[22, \S 19])$.

Il est important de remarquer que la définition de Holim $_{I}$ dépend de la topologie de $C$, et en général, la comparaison entre les limites homotopiques pour différentes topologies n'est pas aisée. On dispose cependant du critère général suivant. Il permettra par la suite d'avoir dans certains cas des décompositions de Postnikov raisonnables, que l'on sait ne pas exister en général.

Lemme 1.2.1. Soit $F: I \longrightarrow S P r(C)$ un $I$-diagramme de préfaisceaux simpliciaux, tel que pour tout $i \in I, F_{i}$ soit un champ. Alors, si on note Holim $_{I}^{\text {triv }} F \in$ $H o(S P r(C))$ la limite homotopique de $F$ lorsque $C$ est muni de la topologie triviale, le morphisme naturel

$$
\mathrm{Holim}_{I}^{\text {triv }} \mathrm{F} \longrightarrow \mathrm{Holim}_{I} \mathrm{~F}
$$

est un isomorphisme dans $\operatorname{Ho}(\operatorname{SPr}(C))$.

Preuve: C'est immédiat, car toute résolution fibrante $F \longrightarrow F^{\prime}$ est telle que les morphismes induits $F_{i} \longrightarrow F_{i}^{\prime}$ soient des équivalences fortes pour tout $i \in I$. Ainsi, $F \longrightarrow F^{\prime}$ est aussi une résolution fibrante lorsque $C$ est muni de la topologie triviale.

Nous allons maintenant étudier deux cas particulier du foncteur précédent. Il s'agit du cas où $I$ possède trois objets 0,1 et 2 et deux uniques morphismes non triviaux $1 \rightarrow 0,2 \rightarrow 0$, et du cas où $I$ est la catégorie associée à l'ensemble totalement ordonné des entiers naturels.

Commençons par examiner le premier cas, et notons 0,1 et 2 les objets de $I$, et $a: 1 \rightarrow 0, b: 2 \rightarrow 0$ ses morphismes non triviaux. Un objet de $\operatorname{SPr}(C)^{I}$ est alors la donnée d'un diagramme de préfaisceaux simpliciaux

$$
F_{1} \stackrel{a}{\longrightarrow} F_{0}<b \quad F_{2} .
$$

Pour tout objet $F \in H o\left(S \operatorname{Pr}(C)^{I}\right)$ nous utiliserons la notation

$$
F_{1} \times F_{0}^{h} F_{2}:=\operatorname{Holim}_{I} F,
$$

que nous appellerons le produit fibré homotopique de $F_{1}$ et $F_{2}$ au-dessus de $F_{0}$. Un cas particulièrement important est celui où $F_{2}=*$. L'objet $F_{1} \times{ }_{F_{0}}^{h} *$ est alors la fibre homotopique du morphisme $a: F_{1} \longrightarrow F_{0}$ au point $b: * \longrightarrow F_{0}$.

Il est facile de vérifier que les objets fibrants dans $\operatorname{SPr}(C)^{I}$ sont exactement les diagrammes tels que $F_{i}$ soit un champ pour tout $i$, et tels que les morphismes $a$ et $b$ soient des fibrations. En particulier, si $F \in S \operatorname{Pr}(C)^{I}$ est fibrant, pour tout $i$ et tout $X \in C, F_{i}(X)$ est un ensemble simplicial fibrant, et les morphismes 
$a_{X}: F_{1}(X) \rightarrow F_{0}(X), b_{X}: F_{2}(X) \rightarrow F_{0}(X)$ sont des fibrations d'ensembles simpliciaux.

Supposons maintenant que $F \in S \operatorname{Pr}(C)^{I}$ soit fibrant et muni d'un morphisme $s: * \longrightarrow F$, qui induit donc des morphismes $s_{i}: * \longrightarrow F_{i}$, et $s: * \longrightarrow F_{1} \times_{F_{0}}^{h} F_{2}$. Alors, en utilisant les suites exactes longues d'homotopie associées aux produits fibrés homotopiques d'ensembles simpliciaux, on obtient une suite exacte longue de préfaisceaux en groupes sur $C$

$$
\begin{gathered}
\pi_{m+1}^{p r}\left(F_{0}, s_{0}\right) \stackrel{\partial}{\longrightarrow} \pi_{m}^{p r}\left(F_{1} \times{ }_{F_{0}}^{h} F_{2}, s\right) \stackrel{a \times b}{\longrightarrow} \pi_{m}^{p r}\left(F_{1}, s_{1}\right) \times \pi_{m}^{p r}\left(F_{2}, s_{2}\right) \stackrel{a-b}{\longrightarrow} \\
\pi_{m}^{p r}\left(F_{0}, s_{0}\right) \stackrel{\partial}{\longrightarrow} \pi_{m-1}^{p r}\left(F_{1} \times{ }_{F_{0}}^{h} F_{2}, s\right) \stackrel{a \times b}{\longrightarrow} \cdots \\
\cdots \longrightarrow \pi_{1}^{p r}\left(F_{1} \times{ }_{F_{0}}^{h} F_{2}, s\right) \stackrel{a \times b}{\longrightarrow} \pi_{1}^{p r}\left(F_{1}, s_{1}\right) \times \pi_{1}^{p r}\left(F_{2}, s_{2}\right) \stackrel{a-b}{\longrightarrow} \\
\pi_{1}^{p r}\left(F_{0}, s_{0}\right) \stackrel{\partial}{\longrightarrow} \pi_{0}^{p r}\left(F_{1} \times_{F_{0}}^{h} F_{2}\right) \stackrel{a \times b}{\longrightarrow} \pi_{0}^{p r}\left(F_{1}\right) \times \pi_{0}^{p r}\left(F_{2}\right) \stackrel{a-b}{\longrightarrow} \pi_{0}^{p r}\left(F_{0}\right) .
\end{gathered}
$$

Par convention, une suite de morphismes d'ensembles $M \stackrel{u}{\longrightarrow} N \stackrel{a-b}{\longrightarrow} P$ est exacte lorsque l'image de $u$ est exactement l'équaliseur des morphismes $a$ et $b$. De même, dire qu'une suite de morphismes d'ensembles pointés $M \stackrel{u}{\longrightarrow} N \stackrel{v}{\longrightarrow} P$ est exacte signifie que l'image de $u$ est égale à la fibre de $v$ au point distingué.

En utilisant l'exactitude du foncteur de faisceautisation, on en déduit une suite exacte longue sur les faisceaux d'homotopie

$$
\begin{gathered}
\pi_{m+1}\left(F_{0}, s_{0}\right) \stackrel{\partial}{\longrightarrow} \pi_{m}\left(F_{1} \times{ }_{F_{0}}^{h} F_{2}, s\right) \stackrel{a \times b}{\longrightarrow} \pi_{m}\left(F_{1}, s_{1}\right) \times \pi_{m}\left(F_{2}, s_{2}\right) \stackrel{a-b}{\longrightarrow} \\
\pi_{m}\left(F_{0}, s_{0}\right) \stackrel{\partial}{\longrightarrow} \pi_{m-1}\left(F_{1} \times{ }_{F_{0}}^{h} F_{2}, s\right) \stackrel{a \times b}{\longrightarrow} \cdots \\
\cdots \longrightarrow \pi_{1}\left(F_{1} \times{ }_{F_{0}}^{h} F_{2}, s\right) \stackrel{a \times b}{\longrightarrow} \pi_{1}\left(F_{1}, s_{1}\right) \times \pi_{1}\left(F_{2}, s_{2}\right) \stackrel{a-b}{\longrightarrow} \\
\pi_{1}\left(F_{0}, s_{0}\right) \stackrel{\partial}{\longrightarrow} \pi_{0}\left(F_{1} \times_{F_{0}}^{h} F_{2}\right) \stackrel{a \times b}{\longrightarrow} \pi_{0}\left(F_{1}\right) \times \pi_{0}\left(F_{2}\right) \stackrel{a-b}{\longrightarrow} \pi_{0}\left(F_{0}\right) .
\end{gathered}
$$

L'existence de cette suite exacte longue et un analogue du lemme des cinqs impliquent en particulier que l'objet $F_{1} \times{ }_{F_{0}}^{h} F_{2}$ ne dépend pas de la topologie de $C$. En d'autres termes, si $F \in S \operatorname{Pr}(C)^{I}$ est un objet, et si on note $F_{1} \times{ }_{F_{0}}^{h f} F_{2}$ le produit fibré homotopique lorsque $C$ est muni de la topologie triviale, alors le morphisme naturel dans $H o(S \operatorname{Pr}(C)), F_{1} \times{ }_{F_{0}}^{h f} F_{2} \longrightarrow F_{1} \times{ }_{F_{0}}^{h} F_{2}$ est un isomorphisme. Une conséquence remarquable de ce fait est que la catégorie de modèles $\operatorname{SPr}(C)$ est propre à droite (i.e. les changements de bases le long de fibrations préservent les équivalences). Pour plus de références sur ce point le lecteur pourra consulter [23, $\S 3]$.

Supposons maintenant que $I$ soit la catégorie définie par l'ensemble ordonné des entiers naturels. Ses objets sont donc les entiers $n \in \mathbb{N}$, et il existe un unique 
morphisme non trivial de $n$ vers $m$ si et seulement si $n>m$. La catégorie $I$ peut donc se représenter schématiquement par

$$
\ldots m \longrightarrow m-1 \longrightarrow \ldots 1 \longrightarrow 0
$$

et un objet de $\operatorname{SPr}(C)^{I}$ est donc la donnée pour tout entier $n$ d'un préfaisceau simplicial $F_{n} \in S \operatorname{Pr}(C)$, et de morphismes $f_{n}: F_{n} \longrightarrow F_{n-1}$. La limite homotopique de $F \in S \operatorname{Pr}(C)^{I}$ sera alors notée $\operatorname{Holim}_{n} F_{n}$.

Comme le foncteur de faisceautisation ne commute pas avec les limites infinies, on ne disposera pas en général d'une suite exacte de Milnor reliant les faisceaux d'homotopie de $\operatorname{Holim}_{n} F_{n}$ avec ceux de $F$. Ceci entraine aussi qu'il n'existe pas de décomposition de Postnikov en général. Nous allons cependant utiliser le lemme 1.2.1 et la suite exacte de Milnor de [15, Prop. VI.2.15] pour donner un critère de convergence des décompositions de Postnikov. Pour cela nous supposerons connu le cas absolu (tel qu'il est décrit dans $[15, \S V I]$ par exemple).

Soit $* \longrightarrow F$ un préfaisceau simplicial pointé tel que $\pi_{0}(F) \simeq *$. Quitte à se restreindre au sous-préfaisceau simplicial de $F$ des simplexes au-dessus du point $*$ (ce qui changera $F$ en un préfaisceau simplicial équivalent), on pourra même supposer que $F$ est 0 -réduit, et donc que $\pi_{0}^{p r}(F) \simeq *$. De plus, quitte à prendre un remplacement fibrant pour la structure forte on pourra supposer que $F(X)$ est un ensemble simplicial fibrant pour tout objet $X \in C$. Rappelons que le préfaisceau en groupes $\pi_{1}^{p r}(F, *)$ opère naturellement sur $\pi_{n}^{p r}(F, *)$, et de même le faisceau en groupes $\pi_{1}(F, *)$ opère sur $\pi_{n}(F, *)$. Nous utiliserons alors les préfaisceaux simpliciaux $K\left(\pi_{1}^{p r}(F, *), \pi_{n}^{p r}(F, *), n+1\right)$ définis dans le paragraphe suivant. Ce sont des préfaisceaux simpliciaux pointés, connexes et fibrants pour la structure forte, et avec

$$
\begin{gathered}
\pi_{i}^{p r}\left(K\left(\pi_{1}^{p r}(F, *), \pi_{n}^{p r}(F, *), n+1\right), *\right) \simeq * \text { pour } i \neq 1, n+1 \\
\pi_{1}^{p r}\left(K\left(\pi_{1}^{p r}(F, *), \pi_{n}^{p r}(F, *), n+1\right), *\right) \simeq \pi_{1}^{p r}(F, *) \\
\pi_{n+1}^{p r}\left(K\left(\pi_{1}^{p r}(F, *), \pi_{n}^{p r}(F, *), n+1\right), *\right) \simeq \pi_{n}^{p r}(F, *),
\end{gathered}
$$

et dont l'action du $\pi_{1}^{p r}$ sur le $\pi_{n}^{p r}$ est celle donnée précédemment.

Considérons la tour de Postnikov de $F,\left\{\tau_{\leq n} F\right\}_{n}$, définie objet par objet de $C$ comme dans [15, VI.3]. Il s'agit d'un diagramme

$$
\cdots \longrightarrow \tau_{\leq n} F \stackrel{p_{n}}{\longrightarrow} \tau_{\leq n-1} F \stackrel{p_{n-1}}{\longrightarrow} \ldots \tau_{\leq 0} F=*,
$$

où la fibre homotopique du morphisme $p_{n}$ est équivalente pour la structure forte au préfaisceau simplicial $K\left(\pi_{n}^{p r}(F, *), n\right)$. On peut donc trouver pour $n>1$, un diagramme homotopiquement cartésien pour la structure forte

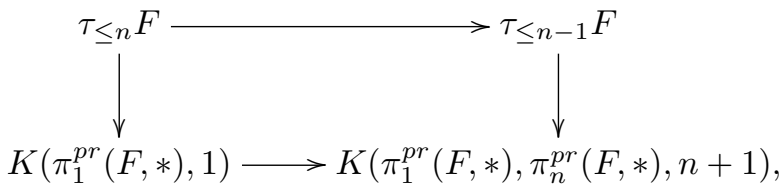


qui est un diagramme classifiant pour la fibration $\tau_{\leq n} F \longrightarrow \tau_{\leq n-1} F$. Mais comme les produits fibrés homotopiques sont indépendants à équivalence près de la topologie sur $C$, on trouve aussi des diagrammes homotopiquement cartésiens

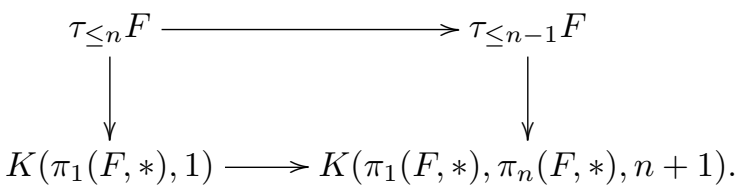

La proposition suivante est due à R. Thomason dans le cas stable, et à J.F. Jardine dans le cas général (voir [28, Lem. 3.4]).

Proposition 1.2.2. Soit $* \longrightarrow F$ un préfaisceau simplicial pointé avec $\pi_{0}(F) \simeq *$, et $E_{n}$ un modèle fibrant pour $K\left(\pi_{n}(F, *), n+1\right)$. Supposons qu'il existe un entier $N$, tel que pour tout entier $i \geq 0$ et tout $n>i+N$ on ait

$$
\pi_{i}^{p r}\left(E_{n}, *\right) \simeq 0 \text {. }
$$

Alors le morphisme naturel

$$
F \longrightarrow \mathrm{Holim}_{n} \tau_{\leq n} F
$$

est un équivalence.

Preuve: Considérons le diagramme commutatif

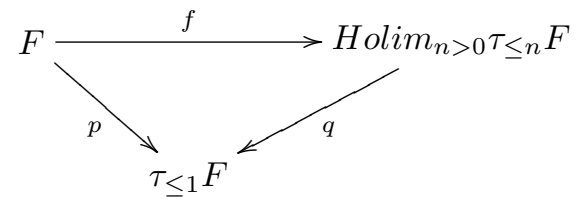

Comme $\operatorname{Holim}_{n>0} \tau_{<n} F$ est naturellement équivalent à $\operatorname{Holim}_{n} \tau_{\leq n} F$, il suffit de montrer que le morphisme $f$ est une équivalence. De plus, une utilisation de la suite exacte longue en homotopie montre qu'il suffit de démontrer que le morphisme induit sur les fibres homotopiques des morphismes $p$ et $q$ est une équivalence. Ceci implique que l'on peut remplacer $F$ par la fibre homotopique de $p$, et donc se ramener au cas où $F$ est simplement connexe (i.e. $\pi_{1}(F, *) \simeq \pi_{0}(F) \simeq *$ ).

On peut clairement supposer que $F$ est un champ, ce que nous ferons. De plus, quitte à remplacer le diagramme $\left\{\tau_{\leq n} F_{n}\right\}_{n}$ par un diagramme qui lui est équivalent, on peut aussi supposer que chaque $\tau_{\leq n} F$ est un champ, et que chacun des diagrammes

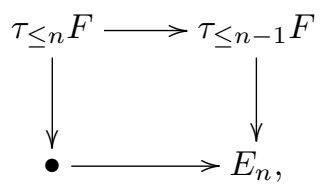


est homotopiquement cartésien pour la structure forte. D'après [15, Prop. VI.2.15], il existe pour tout $i \geq 0$ d'une suite exacte courte de préfaisceaux

$$
\operatorname{Lim}_{n}^{1} \pi_{i+1}^{p r}\left(\tau_{\leq n} F\right) \longrightarrow \pi_{i}^{p r}\left(\operatorname{Holim}_{n}^{\text {triv }} \tau_{\leq n} F\right) \longrightarrow \operatorname{Lim}_{n} \pi_{i}^{p r}\left(\tau_{\leq n} F\right) \longrightarrow 0
$$

où Holim $^{\text {triv }}$ désigne la limite homotopique pour la topologie triviale (et où nous ne mentionons pas le point de base). Cependant, l'hypothèse faite sur les préfaisceaux d'homotopie de $E_{n}$ entraine que le système projectif $\left\{\pi_{i}^{p r}\left(\tau_{\leq n} F, *\right)\right\}_{n}$ est stationnaire. La suite exacte précédente se réduit donc un isomorphisme de préfaisceaux

$$
\pi_{i}^{p r}\left(\operatorname{Holim}_{n}^{\text {triv }} \tau_{\leq n} F, *\right) \simeq \pi_{i}^{p r}\left(\tau_{\leq n} F, *\right),
$$

pour $n>i+N$. En passant aux faisceaux d'homotopie on en déduit que le morphisme naturel $\operatorname{Holim}_{n}^{\text {triv }} \tau_{\leq n} F \longrightarrow \tau_{\leq n} F$ induit des isomorphismes de faisceaux

$$
\pi_{i}\left(\operatorname{Holim}_{n}^{\text {triv }} \tau_{\leq n} F\right) \simeq \pi_{i}\left(\tau_{\leq n} F, *\right),
$$

pour $n>i+N$. Or, comme on a $\pi_{i}(F, *) \simeq \pi_{i}\left(\tau_{\leq n} F\right.$,*) dès que $i \leq n$, ceci implique clairement que le morphisme $F \longrightarrow \operatorname{Holim}_{n}^{\text {triv }} \tau_{\leq n} F$ est une équivalence. Le lemme 1.2.1 implique alors qu'il en est de même du morphisme naturel $F \longrightarrow$ $\operatorname{Holim}_{n} \tau_{\leq n} F$.

Remarque: Remarquer que la condition $\pi_{i}^{p r}\left(E_{n}\right)=0$ pour $n>i+N$ est équivalent à la condition que $H^{n+1-i}\left(X, \pi_{n}(F, *)\right)=0$, pour tout $n>i+N$ et tout objet du site $X \in C$.

En corollaire de la démonstration on trouve le résultat suivant.

\section{Corollaire 1.2.3. Soit}

$$
F \longrightarrow \cdots \longrightarrow F_{n} \longrightarrow F_{n-1} \longrightarrow \cdots \longrightarrow F_{1} \longrightarrow F_{0}=*
$$

une tour de champs pointés et connexes, telle que pour tout $n>0$, la fibre homotopique de $F_{n} \longrightarrow F_{n-1}$ soit équivalente à un préfaisceau simplicial de la forme $K\left(\pi_{n}, n\right)$. Supposons de plus qu'il existe un entier $N>0$ tel que pour tout objet $X \in C$ et tout $n>0$ on ait $H^{n+i-1}\left(X, \pi_{n}\right)=0$ dès que $n>i+N$. Alors, on $a$

$$
\pi_{0}\left(\operatorname{Holim}_{n} F_{n}\right)=* \quad \pi_{i}\left(\operatorname{Holim}_{n} F_{n}, *\right) \simeq \pi_{i} \text { pour } i>0 .
$$

\subsection{Cohomologie des préfaisceaux simpliciaux}

Nous considèrerons essentiellement le cas particulier des préfaisceaux simpliciaux pointés $s: * \longrightarrow F$ tels que le morphisme induit sur le faisceau des composantes connexes $* \longrightarrow \pi_{0}(F)$ soit un isomorphisme. Un tel $F$ sera appelé un préfaisceau simplicial pointé et connexe.

Définition 1.3.1. Un système local $M$ sur un préfaisceau simplicial pointé et connexe $F$, est la donnée d'un faisceau en groupes abéliens $M$, et d'une action de $\pi_{1}(F, s)$ sur $M$. Un morphisme entre deux tels systèmes locaux $M$ et $M^{\prime}$, est la donnée d'un morphisme de faisceaux de groupes $M \longrightarrow M^{\prime}$ qui commute avec les actions de $\pi_{1}(F, s)$. 
La catégorie abélienne des systèmes locaux sur $F$ ainsi définie sera notée $\operatorname{Sys} \operatorname{Loc}(F)$.

Rappelons que pour un $\mathbb{V}$-groupe $G$ on peut construire son classifiant $K(G, 1)$, qui est un $\mathbb{V}$-ensemble simplicial défini de la façon suivante. On commence par définir $E(G, 1)$, qui est l'ensemble simplicial nerf du morphisme naturel $G \longrightarrow *$. Ainsi, $E(G, 1)_{m}:=G^{m+1}$, et les faces et dégénérescences sont données par les projections et les diagonales. Le groupe $G$ opère sur $E(G, 1)$ en opèrant sur luimême à gauche. On définit alors $K(G, 1):=E(G, 1) / G$. Cette construction s'étend naturellement au cas où $G$ est un $\mathbb{V}$-ensemble simplicial muni d'une loi de groupe. En effet, on forme alors l'ensemble bisimplicial $K(G, 1)_{p, q}:=K\left(G_{p}, 1\right)_{q}$, et on définit $K(G, 1)$ comme son ensemble simplicial diagonal. Ainsi, on a $K(G, 1)_{n}=$ $K\left(G_{n}, 1\right)_{n}$. On peut aussi commencer par former l'ensemble bisimplicial nerf du morphisme $G \longrightarrow *$, et appeler sa diagonale $E(G, 1)$. Le groupe $G$ opère alors sur $E(G, 1)$ et on a $K(G, 1)=E(G, 1) / G$. Rappelons aussi que l'ensemble simplicial $K(G, 1)$ est naturellement pointé (par l'image de l'identité de $E(G, 1)$ ), et qu'il existe des isomorphismes naturels $\pi_{m}(K(G, 1), *) \simeq \pi_{m-1}(G, e)$.

Lorsque $G$ est un groupe abélien simplicial l'ensemble simplicial $K(G, 1)$ hérite d'une loi de groupe abélien induite par celle de $G$. On peut alors itérer la construction précédente et poser

$$
E(G, m):=E(K(G, m-1), 1) \quad K(G, m):=K(K(G, m-1), 1) .
$$

La fonctorialité des constructions précédentes permet de définir pour tout préfaisceau en groupes simpliciaux $G$ sur $C$, des préfaisceaux simpliciaux $E(G, 1)$ et $K(G, 1)$. De plus, lorsque $G$ est abélien on dispose aussi des préfaisceaux simpliciaux $E(G, m)$ et $K(G, m)$. Le groupe simplicial $K(G, m-1)$ opère sur $E(G, m)$ et on a $K(G, m)=E(G, m) / K(G, m-1)$. Nous définirons par convention $K(G, 0)$ comme étant le faisceau de groupes simpliciaux $G$.

Soit $G$ un préfaisceau en groupes opèrant sur un préfaisceau en groupes abéliens $M$ à travers un morphisme de préfaisceaux $\rho: G \longrightarrow \underline{A u t}_{C}(M)$. Alors le groupe $G$ opère encore de façon naturelle sur les préfaisceaux en groupes abéliens simpliciaux $K(M, m-1)$, pour $m>0$. On peut donc former le produit semidirect de $G$ par $K(M, m-1)$, qui est un préfaisceau en groupes simpliciaux noté $G \times{ }_{\rho} K(M, m-1)$. On notera par la suite $K(G, M, m):=K\left(G \times{ }_{\rho} K(M, m-1), 1\right)$. On dispose de plus d'une suite exacte de préfaisceaux en groupes simpliciaux

$$
K(M, m-1) \longrightarrow G \times{ }_{\rho} K(M, m) \longrightarrow G .
$$

En en prenant l'image par le foncteur $K(-, 1)$ on en déduit deux nouveaux morphismes

$$
K(M, m) \longrightarrow K(G, M, m) \stackrel{p}{\longrightarrow} K(G, 1) .
$$

Pour $m=0$, on définit par convention $K(G, M, 0)$ comme étant le préfaisceau en groupes $G \times{ }_{\rho} M$, produit semi-direct de $G$ par $M$. La suite exacte longue en homotopie implique alors que $K(M, m)$ est naturellement équivalent dans $S \operatorname{Pr}(C)$ 
à la fibre homotopique du morphisme $p$, et ceci pour tout $m \geq 0$. Le préfaisceau simplicial $K(G, M, m)$ est donc caractérisé à équivalence forte près par le fait que

$$
\begin{gathered}
\pi_{i}^{p r}(K(G, M, m), *)=* \text { pour } i \neq 1, m \\
\pi_{1}^{p r}(K(G, M, m), *) \simeq G \pi_{m}^{p r}(K(G, M, m), *) \simeq M,
\end{gathered}
$$

et par l'action de $G$ sur $M$.

Soit maintenant $F$ un préfaisceau simplicial connexe, et $\rho: G=\pi_{1}(F, s) \longrightarrow$ $\underline{A u t}_{C}(M)$ un système local. On dispose d'un morphisme d'ensembles simpliciaux

$$
p^{*}: \mathbb{R} \underline{\operatorname{Hom}}(F, K(G, M, m)) \longrightarrow \mathbb{R} \underline{\operatorname{Hom}}(F, K(G, 1)) .
$$

De plus, il existe un morphisme naturel dans $H o(S \operatorname{Pr}(C)), F \longrightarrow \tau_{\leq 1} F \simeq K(G, 1)$, qui définit donc un point $* \in \mathbb{R} \underline{\operatorname{Hom}}(F, K(G, 1))$. Nous définissons alors l'ensemble simplicial $\mathbb{R} \underline{\operatorname{Hom}}_{\rho}(F, K(M, m))$ comme étant la fibre homotopique du morphisme $p^{*}$ au point $*$. Remarquer que si $\rho$ est le morphisme trivial alors l'ensemble simplicial $\mathbb{R} \underline{\operatorname{Hom}}_{\rho}(F, K(M, m))$ est naturellement équivalent à $\mathbb{R} \underline{\operatorname{Hom}}(F, K(M, m))$.

Définition 1.3.2. Le $m$-ème groupe de cohomologie du préfaisceau simplicial pointé et connexe $F$, à coefficients dans le système local $\rho: \pi_{1}(F, s) \longrightarrow \underline{A u t}_{C}(M)$ est défini par

$$
H^{m}(F, M):=\pi_{0}\left(\mathbb{R}_{\text {Hom }_{\rho}}(F, K(M, m))\right) .
$$

Plus généralement, si $F \in S \operatorname{Pr}(C)$ est un préfaisceau simplicial (non pointé) et $M$ un faisceau de groupes abéliens sur $C$, nous noterons

$$
H^{m}(F, M):=\pi_{0} \underline{\mathbb{R} \underline{H o m}}(F, K(M, m)) .
$$

Lorsque $F=h_{X}$, pour $X$ un objet du site $C$, ces groupes seront notés $H^{m}(X, M)$, et $H^{m}(C, M)$ si $X$ est l'objet final de $C$.

En utilisant les suites exactes longues en homotopie associées à des fibrations, il est facile de vérifier les deux faits suivants.

- Le groupe $H^{0}(F, M)$ s'identifie naturellement au sous-groupe de $\Gamma(M)$ invariant par l'action de $\Gamma\left(\pi_{1}(F, s)\right)$.

- Pour toute suite exacte $0 \longrightarrow M \longrightarrow M^{\prime} \longrightarrow M^{\prime \prime} \longrightarrow 0$ de systèmes locaux, il existe une suite exacte longue fonctorielle

$$
\begin{gathered}
0 \longrightarrow H^{0}(F, M) \longrightarrow H^{0}\left(F, M^{\prime}\right) \longrightarrow H^{0}\left(F, M^{\prime \prime}\right) \longrightarrow H^{1}(F, M) \longrightarrow \cdots \\
H^{m}(F, M) \longrightarrow H^{m}\left(F, M^{\prime}\right) \longrightarrow H^{m}\left(F, M^{\prime \prime}\right) \longrightarrow H^{m+1}(F, M) \longrightarrow \cdots
\end{gathered}
$$

Lorsque $(F, s)=(K(G, 1), *)$, pour $G$ un préfaisceau en groupes sur $C$, les foncteurs $H^{m}(F, M)$ sont en réalité des foncteurs dérivés. Comme nous ne connaissons pas de références générales sur le sujet nous donnerons une esquisse de preuve du théorème suivant. Il s'agit de toute évidence d'un résultat faisant partie du folklore de la théorie. 
Théorème 1.3.3. Soit $G$ un préfaisceau en groupes $\operatorname{sur} C$ et $(F, s)=(K(G, 1), *)$. Alors, pour tout entier $m$, le foncteur

$$
\begin{array}{rllc}
H^{m}(F,-): & \operatorname{SysLoc}(F) & \longrightarrow & \mathbb{V}-A b \\
(\rho: G \rightarrow M) & \mapsto & H^{m}(F, M)
\end{array}
$$

est isomorphe au m-ème foncteur dérivé du foncteur $H^{0}(F,-)$.

Esquisse de preuve: Commençons par traiter le cas où $G=\{1\}$ est trivial. Il faut alors montrer que pour tout faisceau de groupes abéliens $M \operatorname{sur} C, H^{m}(C, M)$ est isomorphe à la cohomologie de $C$, calculée par foncteur dérivé, à coefficients dans $M$ (que nous noterons $H_{d e r}^{m}(C,-)$ ). Pour cela, notons $C^{-}(C, A b)$ la catégorie des complexes de préfaisceaux en $\mathbb{V}$-groupes abéliens sur $C$ qui sont concentrés en degrés négatifs ou nuls (avec une différentielle de degré +1 ). Pour chaque objet $E$ de cette catégorie, on dispose de ses préfaisceaux de cohomologie $\underline{H}_{p r}^{n}(E)$, dont les faisceaux associés seront notés $\underline{H}^{n}(E)$. Un quasi-isomorphisme est par définition un morphisme de $C^{-}(C, A b), f: E \longrightarrow E^{\prime}$, tel que pour tout $m$, le morphisme induit $H^{m}(f): \underline{H}^{m}(E) \longrightarrow \underline{H}^{m}\left(E^{\prime}\right)$ soit un isomorphisme de faisceaux. On notera alors $D^{-}(C, A b)$ la catégorie obtenue à partir de $C^{-}(C, A b)$ en inversant formellement les quasi-isomorphismes. Il est alors bien connu que les foncteurs de cohomologie par foncteurs dérivés se calculent par la formule suivante

$$
H_{d e r}^{m}(C, M) \simeq[\underline{\mathbb{Z}}, M[m]]_{D^{-}(C, A b)},
$$

où $\underline{\mathbb{Z}}$ est le préfaisceau constant de fibre $\mathbb{Z}$, et $M[m]$ est le complexe concentré en degré $-m$ et de valeurs $M$.

Introduisons $S A b(C)$, la catégorie des préfaisceaux en $\mathbb{V}$-groupes abéliens simpliciaux sur $C$. D'après [15, Cor. $I I I .2 .3]$ il existe une équivalence de catégories $\Gamma: C^{-}(C, A b) \longrightarrow S A b(C)$ (où $\Gamma$ est défini terme à terme au-dessus de $C$ ). De plus, en remplaçant les ensembles simpliciaux par des groupes abéliens simpliciaux dans les paragraphes précédents, on munit $S A b(C)$ d'une structure de catégorie de modèles où les équivalences et les fibrations sont detectées dans $S \operatorname{Pr}(C)$ (i.e. en oubliant la structure de groupes). A l'aide de [15, Cor. III.2.7] on voit que le foncteur $\Gamma$ est compatible avec la formation des faisceaux de cohomologie et d'homotopie, et qu'il définit une bijection de l'ensemble des quasi-isomorphismes de $C^{-}(C, A b)$ vers l'ensemble des équivalences de $S A b(C)$. Il induit donc une équivalence sur les catégories homotopiques associées

$$
\Gamma: D^{-}(C, A b) \simeq H o(S A b(C)) .
$$

Ainsi, pour tout préfaisceau en groupes abéliens $M$ on a

$$
[\underline{\mathbb{Z}}, M[m]]_{D^{-}(C, A b)} \simeq[\Gamma(\underline{\mathbb{Z}}), \Gamma(M[m])]_{H o(S A b(C))} .
$$

Cependant $\Gamma(\underline{\mathbb{Z}})$ est équivalent dans $S A b(C)$ au préfaisceau constant de fibre $\mathbb{Z}$, et $\Gamma(M[m])$ est équivalent à $K(M, m)$. On a donc

$$
H_{d e r}^{m}(C, M) \simeq[\underline{\mathbb{Z}}, M[m]]_{H o\left(C^{-} A b(C)\right)} \simeq[\underline{\mathbb{Z}}, K(M, m)]_{H o(S A b(C))} .
$$


Pour achever la démonstration dans ce premier cas, on fait appel à l'adjonction de Quillen

$$
-\otimes \mathbb{Z}: S \operatorname{Pr}(C) \longrightarrow S A b(C) \quad j: S A b(C) \longrightarrow S \operatorname{Pr}(C),
$$

où $-\otimes \mathbb{Z}$ est le foncteur d'abélianisation, et $j$ est le foncteur d'oublie de la structure de groupes. Cette adjonction descend en une adjonction sur les catégories homotopiques, et donne

$$
\begin{aligned}
H_{d e r}^{m}(C, M) & \left.\simeq[\underline{\mathbb{Z}}, K(M, m)]_{H o(S A b(C))} \simeq[*, j(K(M, m))]_{H o(S P r}(C)\right) \\
& \simeq \pi_{0} \mathbb{R} \underline{\operatorname{Hom}}(*, K(M, m)) \simeq H^{m}(C, M) .
\end{aligned}
$$

Revenons au cas général où $F=K(G, 1)$, pour $G$ un préfaisceau en groupes. Rappelons que l'on peut définir un site $G$-équivariant $C / B G$. Ses objets sont ceux de $C$, et ils seront notés symboliquement $X \longrightarrow B G$ pour $X \in C$. La donnée d'un morphisme dans $C / B G$

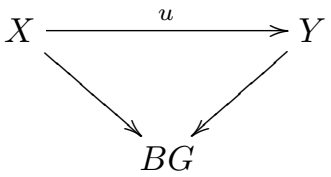

est la donnée d'un couple $(f, x)$, où $f: X \rightarrow Y$ est un morphisme de $C$, et $x \in G(X)$. Les morphismes se composent naturellement par la formule $(g, y) \circ$ $(f, x)=\left(g \circ f, f^{*}(y) . x\right)$, pour $X \stackrel{f}{\longrightarrow} Y \stackrel{g}{\longrightarrow} Z$ et $x \in G(X), y \in G(Y)$. La topologie sur $C / B G$ est définie en décrétant que les familles couvrantes dans $C / B G$ sont celles qui sont couvrantes dans $C$. Il est clair que $C / B G$ possède des produits fibrés, mais il ne possède pas de produits finis en général (et encore moins d'objet final). De plus, la donnée d'un préfaisceau (resp. d'un faisceau) sur $C / B G$ est naturellement équivalente à celle d'un préfaisceau (resp. d'un faisceau) sur $C$ muni d'une action de $G$. Il existe donc une équivalence de catégorie $\operatorname{SPr}(C / B G) \simeq$ $G-S \operatorname{Pr}(C)$, où $G-S P r(C)$ est la catégorie des objets de $S P r(C)$ munis d'une action de $G$. Ceci montre aussi que la catégorie abélienne $\operatorname{Sys} \operatorname{Loc}(K(G, 1))$ est équivalente à la catégorie des faisceaux en groupes abéliens sur $C / B G$, et donc possède suffisemment d'injectifs.

Rappelons aussi que pour tout objet $F \in S \operatorname{Pr}(C)$, on peut considérer la catégorie des objets sur $F, S \operatorname{Pr}(C) / F$, qui est naturellement munie d'une structure de catégorie de modèles fermée simpliciale. De plus, pour tout morphisme $f: F \longrightarrow$ $F^{\prime}$ on dispose d'une adjonction de Quillen

$$
\begin{array}{cccc}
f_{!}: & S \operatorname{Pr}(C) / F & \longrightarrow & S \operatorname{Pr}(C) / F^{\prime} \\
(G \rightarrow F) & \mapsto & \left(G \rightarrow F \rightarrow F^{\prime}\right) \\
f^{*}: & S \operatorname{SPr}(C) / F^{\prime} & \longrightarrow & S \operatorname{Pr}(C) / F \\
& \left(G \rightarrow F^{\prime}\right) & \mapsto & \left(F \times{ }_{G} F^{\prime} \rightarrow F\right),
\end{array}
$$

où $f_{\text {! }}$ est l'adjoint à gauche. Comme $\operatorname{SPr}(C)$ est une catégorie de modèles propre à droite, l'adjonction précédente est en réalité une équivalence de Quillen dès que $f$ est une équivalence. 
Notons alors $\underline{\operatorname{Hom}}_{F}$ les $H$ om simpliciaux de $S \operatorname{Pr}(C) / F$. On vérifie à l'aide des définitions et de l'équivalence de Quillen ci-dessus, que pour tout système local $\rho: G \longrightarrow \underline{A u t}_{C}(M)$ sur $K(G, 1)$, on dispose d'isomorphismes naturels

$$
H^{m}(K(G, 1), M) \simeq \pi_{0} \underline{\mathbb{R o m}}_{K(G, 1)}(K(G, 1), K(G, M, m)) .
$$

En utilisant un argument tout à fait similaire à celui utilisé dans $[57, \S 2.3]$ on peut démontrer qu'il existe une équivalence de Quillen de la catégorie de modèles $S \operatorname{Pr}(C) / K(G, 1)$ vers la catégorie de modèles $S \operatorname{Pr}(C / B G)$ (on renvoit à [31] pour plus de détails sur cette équivalence. Lorsque $G$ n'est pas un préfaisceau simplicial cofibrant on en prendra tout d'abord un remplacement cofibrant en tant que préfaisceau en groupes simpliciaux). Ainsi, on en déduit un isomorphisme naturel

$$
H^{m}(K(G, 1), M) \simeq \pi_{0} \underline{\mathbb{H o m}}_{B G}\left(*, K(M, m)_{B G}\right),
$$

où $\underline{H o m}_{B G}$ désigne le Hom simplicial de la catégorie de modèles simpliciale $S \operatorname{Pr}(C / B G)$, et $K(M, m)_{B G}$ est le préfaisceau simplicial sur $C / B G$ correspondant à travers l'équivalence $\operatorname{SPr}(C / B G) \simeq G-S \operatorname{Pr}(C)$ au préfaisceau simplicial $K(M, m)$ muni de son action naturelle. En faisant appel au cas particulier traité précédemment pour le site $C / B G$, on voit que $H_{\rho}^{m}(K(G, 1), M)$ est isomorphe à $H_{d e r}^{m}(C / B G, M)$. En particulier, si $M$ correspond à un objet injectif dans la catégorie des systèmes locaux sur $K(G, 1)$, on a $H^{m}(K(G, 1), M)=0$ pour $m>0$. On en déduit que la collection de foncteurs $\left\{H^{m}(K(G, 1),-)\right\}_{m}$ forme un foncteur cohomologique effaçable, et qu'il est donc isomorphe au foncteur dérivé de $H^{0}(K(G, 1),-)$.

Remarquer que le théorème précédent appliqué au site $C / X$, des objets sur un objet $X$, montre que le foncteur $H^{m}(X,-)$ est le $m$-ème foncteur dérivé du foncteur des sections globales $\Gamma: A b(C / X) \longrightarrow A b$. Ainsi, pour les préfaisceaux simpliciaux représentables par des objets du site, la cohomologie que nous avons définie dans la définition 1.3.2 coincide avec la cohomologie définie par foncteurs dérivés.

\section{4. $H_{\infty}$-Champs}

Dans ce paragraphe nous avons rassemblé quelques résultats de la théorie du délaçage dans le cadre des préfaisceaux simpliciaux. La référence pour le cas topologique est [46].

Soit $s: * \longrightarrow F$ un préfaisceau simplicial pointé. On définit le préfaisceau des lacets de base $s$ par la formule

$$
\begin{aligned}
\Omega_{s} F: & C^{o} \\
X & \mapsto \mathbb{V}-S E n s \\
& \mapsto \Omega_{s(X)} F(X) .
\end{aligned}
$$

Ici, pour un ensemble simplicial pointé $(X, x), \Omega_{x} X$ est défini comme étant l'ensemble simplicial des morphismes de $\Delta^{1}$ vers $X$ qui envoient les deux sommets de $\Delta^{1}$ sur $x$. La définition précédente fournit un foncteur $\Omega: S \operatorname{Pr}(C)_{*} \longrightarrow S \operatorname{Pr}(C)_{*}$ de la catégorie des préfaisceaux simpliciaux pointés vers elle même. On peut vérifier 
que ce foncteur est en réalité de Quillen à droite. Il se dérive donc en un foncteur sur les catégories homotopiques

$$
\mathbb{R} \Omega: H o\left(S P r(C)_{*}\right) \longrightarrow H o\left(S P r(C)_{*}\right) .
$$

A l'aide de la suite exacte longue en homotopie on vérifie aisément que les faisceaux d'homotopie de $\mathbb{R} \Omega_{s} F$ sont donnés par

$$
\pi_{m}\left(\mathbb{R} \Omega_{s} F, *\right) \simeq \pi_{m+1}(F, s) \quad \pi_{0}\left(\mathbb{R} \Omega_{s} F\right) \simeq \pi_{1}(F, s) .
$$

En particulier, ceci montre que l'on peut calculer $\mathbb{R} \Omega_{*} F$ en supposant que $C$ est muni de la topologie triviale. Pour être plus précis, ceci veut dire que si l'on note $\mathbb{R}_{f} \Omega_{*}$ le foncteur dérivé à droite de $\Omega_{*}$ lorsque $C$ est muni de la topologie triviale, alors le morphisme naturel dans $\operatorname{Ho}(S \operatorname{Pr}(C))$

$$
\mathbb{R}_{f} \Omega_{*}(F) \longrightarrow \mathbb{R} \Omega_{*}(F)
$$

est un isomorphisme. Ceci va nous permettre de généraliser sans trop de problèmes les résultats standards de [46] au cas des préfaisceaux simpliciaux.

Définition 1.4.1. Un pré- $\Delta^{o}$-préfaisceau simplicial est un foncteur

$$
\begin{array}{rlc}
F: \Delta^{o} & \longrightarrow & \operatorname{SPr}(C) \\
{[m]} & \mapsto & F_{m}
\end{array}
$$

tel que $F_{0}=*$. La catégorie des pré- $\Delta^{o}$-préfaisceaux simpliciaux sera notée $\operatorname{Pr} \Delta^{o}-$ $S \operatorname{Pr}(C)$.

Cette définition et les résultats qui vont suivre valent en particulier pour le cas où $C=*$ est le site trivial. Nous noterons dans ce cas $\operatorname{Pr}-\Delta^{o}-S E n s:=$ $\operatorname{Pr}-\Delta^{o}-S \operatorname{Pr}(*)$, que nous appellerons la catégorie des pré- $\Delta^{o}$-ensembles simpliciaux.

Comme [0] est initial dans $\Delta^{o}$, on peut identifier la catégorie $\operatorname{Pr} \Delta^{o}-S \operatorname{Pr}(C)$ avec la catégorie des préfaisceaux sur $\Delta-[0]$ à valeurs dans $S \operatorname{Pr}(C)_{*}$, la catégorie des préfaisceaux simpliciaux pointés. Comme $\operatorname{Sr}(C)_{*}$ est une catégorie de modèles engendrée par cofibrations on peut alors appliquer [15, §II.6.9] et munir $\operatorname{Pr} \Delta^{o}-$ $S \operatorname{Pr}(C)$ d'une structure de catégorie de modèles. Rappelons que les fibrations (resp. les équivalences) pour cette structure sont les morphismes $f: G \longrightarrow G$ tels que pour tout $[m] \in \Delta$, le morphisme induit $f_{m}: F_{m} \longrightarrow G_{m}$ soit une fibration (resp. une équivalence) dans $\operatorname{SPr}(C)$.

Soit $F: \Delta^{o} \longrightarrow D$ un objet simplicial d'une catégorie $D$ possédant des produits finis, et supposons que $F_{0}$ soit l'objet final dans $D$. Rappelons que l'on peut alors définir pour tout $n \geq 1$ le $n$-ème morphisme de Segal $F_{n} \longrightarrow F_{1}^{n}$. Il est induit par les $n$-morphismes dans $\Delta, p_{i}:[1] \longrightarrow[n]$, où chaque $p_{i}$ est défini par $p_{i}(0)=i$ et $p_{i}(1)=i+1$.

Définition 1.4.2. Un objet $F$ de $\operatorname{Pr} \Delta^{o}-S \operatorname{Pr}(C)$ est appelé un $H_{\infty}$-champ s'il vérifie les trois conditions suivantes. 
- Pour tout $n, F_{n}$ est un champ.

- Pour tout $n$, le morphisme de Segal

$$
F_{n} \longrightarrow F_{1}^{n}
$$

est une équivalence dans $\operatorname{SPr}(C)$.

- La loi de monoïde naturellement induite sur le faisceau $\pi_{0}\left(F_{1}\right)$ est une loi de groupe.

Par extensions, nous appellerons encore $H_{\infty}$-champ tout objet de la catégorie homotopique $H o\left(\operatorname{Pr}-\Delta^{o}-S \operatorname{Pr}(C)\right)$ qui est isomorphe à un $H_{\infty}$-champ.

Pour un objet $F \in \operatorname{Pr} \Delta^{o}-\operatorname{SPr}(C)$ on définit son préfaisceau simplicial classifiant $B F$, défini par $B F_{n}:=\left(F_{n}\right)_{n}$. Si $F$ est vu comme un préfaisceau bisimplicial sur $C$, alors $B F$ en est le préfaisceau simplicial diagonal. De plus, comme $F_{0}=*, B F$ est un préfaisceau simplicial réduit (i.e. $F_{0}=*$ ), et en particulier peut-être vu comme un préfaisceau simplicial pointé, $B F \in S \operatorname{Pr}(C)_{*}$. Ceci définit évidemment un foncteur

$$
B: \operatorname{Pr} \Delta^{o}-S \operatorname{Pr}(C) \longrightarrow S \operatorname{Pr}(C)_{*} .
$$

Ce foncteur possède un adjoint à droite, noté usuellement $\Omega_{*}$. Pour mémoire rappelons que pour $F \in S \operatorname{Pr}(C)_{*}, \Omega_{*}(F)$ est défini par

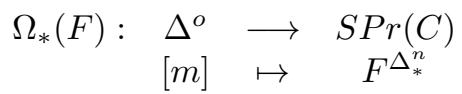

où $\Delta_{*}^{m}$ est l'ensemble simplicial pointé obtenu à partir de $\Delta^{m}$ en identifiant tous ses sommets en un point unique. En d'autres termes, $\Omega_{*} F_{m}$ est le préfaisceau simplicial des morphismes $\Delta^{m} \longrightarrow F$ qui envoient tous les sommets de $\Delta^{m}$ sur le point distingué de $F$.

Le théorème suivant est une généralisation du théorème principal de la théorie du délaçage de G. Segal. Comme nous ne connaissons pas de références précises nous donnerons quelques indications pour montrer qu'il se déduit du théorème original.

Théorème 1.4.3. Le foncteur $\Omega_{*}: S \operatorname{Pr}(C)_{*} \longrightarrow \operatorname{Pr}-\Delta^{o}-S \operatorname{Pr}(C)$ est de Quillen $\grave{a}$ droite, et son adjoint à gauche $B$ préserve les équivalences. De plus les deux assertions suivantes sont vraies.

1. Pour tout objet $F \in S \operatorname{Pr}(C)_{*}, \mathbb{R} \Omega_{*}(F)$ est un $H_{\infty}$-espace, et le morphisme d'adjonction

$$
B \mathbb{R} \Omega_{*} F \longrightarrow F
$$

est un isomorphisme dans $\operatorname{Ho}\left(\operatorname{SPr}(C)_{*}\right)$ lorsque $F$ est connexe (i.e. $\pi_{0}(F) \simeq$ *).

2. De même, si $F$ est un $H_{\infty}$-champ, alors le morphisme d'adjonction

$$
F \longrightarrow \mathbb{R} \Omega_{*}(B F)
$$

est un isomorphisme dans la catégorie homotopique $\mathrm{Ho}\left(\operatorname{Pr}-\Delta^{o}-S \operatorname{Pr}(k)\right)$. 
Esquisse de preuve: Le fait que $\left(B, \Omega_{*}\right)$ soit une adjonction de Quillen pour la structure forte est immédiat. De plus, on vérifie sans problèmes que $\Omega_{*}$ envoie tout objet fibrant sur un objet fibrant. Le fait que le couple de foncteurs $\left(B, \Omega_{*}\right)$ est une adjonction de Quillen est alors une propriété générale des localisations de Bousfield à gauche (On pourra par exemple appliquer [22, Thm. 3.4.20]). Comme $B$ préserve les équivalences fortes et que les fibrations triviales sont aussi des équivalences fortes, il est facile de voir que $B$ préserve aussi les équivalences.

(1) Le fait que $\Omega_{*}(F)$ soit un $H_{\infty}$-champ lorsque $F$ est fibrant provient immédiatement de sa définition et du fait que $\pi_{0}\left(\Omega_{*}(F)\right) \simeq \pi_{1}(F, *)$. Pour voir que le morphisme d'adjonction est un isomorphisme, on peut par exemple utiliser le fait que le foncteur dérivé de $\Omega_{*}$ est indépendant de la topologie sur $C$. Quitte à se restreindre au sous-préfaisceau simplicial de $F$ des simplexes au-dessus du point * on peut ainsi se ramener au cas où la topologie est triviale. Dans ce cas c'est une application de [46] objet par objet de $C$.

(2) Soit $F$ un $H_{\infty}$-champ, que l'on peut supposer fibrant dans $\operatorname{Pr}-\Delta^{o}-$ $S \operatorname{Pr}(C)$. Comme les morphismes de Segal $F_{n} \longrightarrow F_{1}^{n}$ sont des équivalences entre objets fibrants, ce sont des équivalences fortes. De plus, dire que $\pi_{0}(F)$ est un groupe est équivalent à dire que le morphisme $F_{2} \longrightarrow F_{1}^{2}$, donné par la "multiplication" et "la première projection" est une équivalence. De même, c'est une équivalence forte. On voit ainsi que $F$ est un $H_{\infty}$-champ lorsque $C$ est muni de la topologie triviale. De plus, on a vu que le foncteur dérivé $\mathbb{R} \Omega_{*}$ pouvait se calculer en utilisant la topologie triviale. On en déduit donc que l'on peut supposer que $C$ est muni de la topologie triviale. Le résultat découle alors d'une application de [46] objet par objet de $C$.

Tout comme dans [57], on déduit du théorème 1.4.3 une équivalence entre la théorie homotopique des objets en groupes dans $\operatorname{SPr}(C)$ et celle des $H_{\infty^{-}}$ champs sur $C$. Nous utiliserons en particulier que tout $H_{\infty}$-champ est naturellement équivalent à un préfaisceau en groupes simpliciaux.

\subsection{Schémas en groupes affines}

Pour tout ce paragraphe $k$ sera un corps de caractéristique quelconque, et nous noterons par $(A f f / k)_{f p q c}$ le site des U-schémas affines sur Speck muni de la topologie fidèlement plate et quasi-compacte (voir [47, VI.6.3]). Nous noterons alors $\operatorname{SPr}(k)$ la catégorie des préfaisceaux en $\mathbb{V}$-ensembles simpliciaux sur le site de Grothendieck $(A f f / k)_{f p q c}$, munie de sa structure de catégorie de modèles décrite dans le paragraphe 1.1 .

Notons $G p A f f / k$ la catégorie des groupes dans $A f f / k$, dont nous appellerons les objets des schémas en groupes affines (ainsi un schéma en groupes affines sera toujours un élément de $\mathbb{U})$. Tout objet $G \in G p A f f / k$ représente un faisceau en groupes $h_{G}$. En notant $G p(k)$ la catégorie des faisceaux en $\mathbb{V}$-groupes sur 
$(A f f / k)_{f p q c}$, ceci induit un foncteur

$$
\begin{aligned}
h: \quad \text { GpAff/k } & \longrightarrow G p(k) \\
G & \mapsto h_{G},
\end{aligned}
$$

qui par le lemme de Yoneda est pleinement fidèle. Les objets dans l'image essentielle de ce foncteur seront aussi appelés des schémas en groupes affines.

Rappelons alors que le fait que $k$ soit un corps entraine que le foncteur $h_{G}$ est exact (voir $\left[11, I I I, \S 3, n^{o} 7\right]$ ). Ceci signifie que pour toute suite exacte dans GpAff/k

$$
1 \longrightarrow G_{1} \longrightarrow G_{2} \longrightarrow G_{3} \longrightarrow 1
$$

la suite induite

$$
1 \longrightarrow h_{G_{1}} \longrightarrow h_{G_{2}} \longrightarrow h_{G_{3}} \longrightarrow 1
$$

est une suite exacte de faisceaux sur $(A f f / k)_{f p q c}$. En particulier, la sous-catégorie de $G p(k)$ formée des schémas en groupes affines est stable par noyaux, co-noyaux et images. De plus, d'après [39, III Thm. 4.3] on sait que si $G$ est un schéma en groupe affine, $X$ un schéma affine et $P$ un $h_{G}$-torseur de faisceau quotient $h_{X}$, alors $P$ est représentable par un schéma affine. Ainsi, la sous-catégorie pleine de $G p(k)$ formée des schémas en groupes affines est aussi stable par extensions. Enfin, comme le foncteur d'oublie de la structure de groupe commute avec les limites, et qu'une U-limite projective de schémas affines est un schéma affine, on voit que la sous-catégorie pleine de $G p(k)$ des schémas en groupes affines est aussi stable par U-limites projectives. Notons aussi que le faisceau image d'un morphisme de schémas en groupes affines $f: G \longrightarrow H$ est toujours représenté par un sous-schéma fermé dans $H([11, I I I, \S 3, T h m .7 .2])$.

Rappelons enfin qu'un schéma en groupes affine $G$ est la limite projective de ses quotients de types finis sur $k$. Ceci permet alors d'identifier la catégorie des schémas en groupes affines avec celle des Pro-objets (dans $\mathbb{U}$ ) dans la catégorie des schémas en groupes affines de type fini sur $k$ (voir $[11, I I I, \S 3$, Cor.7.5]).

Ces propriétés nous permettent d'identifier sans risque un schéma en groupes affine $G$ au faisceau qu'il représente $h_{G}$. Ainsi, par la suite nous noterons $G$ aussi bien pour désigner l'un ou l'autre.

Nous appellerons représentation linéaire d'un schéma en groupes affine $G$, la donnée d'un $G-\mathcal{O}$-module dont le $\mathcal{O}$-module sous-jacent est quasi-cohérent et appartient à $\mathbb{U}$ (voir $[11, I I, \S 2])$. C'est donc la donnée d'un $k$-espace vectoriel $V$ (éventuellement de dimension infini) de $\mathbb{U}$, et pour tout schéma affine $\operatorname{Spec} A \in$ Aff $/ k$ d'un morphisme de groupes $G(A) \longrightarrow A u t_{A}(V \otimes A)$, fonctoriel en $A$. La catégorie des représentations linéaires de $G$ sera notée $\operatorname{Rep}_{k}(G)$. Pour $V$ une telle représentation, nous noterons encore par $V$ le faisceau de $G-\mathcal{O}$-modules sousjacent. Notons aussi que toute représentation linéaire est limite inductive de ses sous-représentations de dimension finie sur $k$. Ainsi, la catégorie $\operatorname{Rep}_{k}(G)$ s'identifie à la catégorie des Ind-objets de la catégorie des représentations linéaires sur $G$ de dimension finie. Remarquons enfin qu'une représentation linéaire $V$ de dimension 
finie $d$, d'un schéma en groupes affine $G$, détermine une action de $G$ sur le schéma en groupes affine $V \simeq \mathbb{G}_{a}^{d}$.

L'oublie de la structure de $\mathcal{O}$-module permet de voir une représentation linéaire de $G$ comme un système local sur $K(G, 1)$ (voir Def. 1.3.1). Ceci fournit un foncteur $\operatorname{Rep}_{k}(G) \longrightarrow \operatorname{Sys} \operatorname{Loc}(K(G, 1))$, qui est exact et fidèle, mais qui n'est pas pleinement fidèle en général. Ce foncteur nous permet de parler de cohomologie d'un schéma en groupes affine $G$ à valeurs dans une représentation linéaire $\mathbb{V}$. Par définition il s'agira de la cohomologie de $K(G, 1)$ à valeurs dans le système local correspondant. Ces groupes de cohomologie seront simplement notés $H^{i}(K(G, 1), V)$, et sont naturellement munis d'une structure de $k$-espaces vectoriels. Remarquer que $H^{0}(K(G, 1), V)$ s'identifie naturellement au sous- $k$-espace vectoriel de $V$ des points fixes par $G$, qui sera noté par la suite $V^{G}$.

Lemme 1.5.1. Pour tout entier $i$, le foncteur

$$
H^{i}(K(G, 1),-): \operatorname{Rep}_{k}(G) \longrightarrow k-V e c t
$$

est le $i$-ème foncteur dérivé du foncteur $H^{0}(K(G, 1),-)$.

Esquisse de preuve: Soit $V \in \operatorname{Rep}_{k}(G)$ une représentation linéaire de $G$. Le faisceau sous-jacent au système local $V \in \operatorname{Sys} \operatorname{Loc}(K(G, 1))$ étant quasi-cohérent il est acyclique sur $(A f f / k)_{f p q c}$. Le préfaisceau simplicial $K(V, i)$ est donc fibrant en tant qu'objet de la catégorie $G-S \operatorname{Pr}(k)$, des préfaisceaux simpliciaux munis d'une action de $G$ (ou encore des préfaisceaux simpliciaux sur le site équivariant $(A f f / k) / B G)$. La cohomologie de $K(G, 1)$ à valeurs dans $V$ peut donc se calculer à l'aide de la topologie triviale sur $A f f / k$. Mais alors, on sait d'après le théorème 1.3.3 que le foncteur $H^{i}(K(G, 1),-)$ est le $i$-ème foncteur dérivé du foncteur des sections globales sur la catégorie des préfaisceaux en groupes abéliens sur $\mathrm{Af} f / k$, munis d'une action de $G$. Ainsi, d'après la proposition [47, $I$ Prop. 5.2.1] appliquée au préfaisceau d'anneaux constant $\mathbb{Z}$, on sait alors que $H^{i}(K(G, 1), V)$ est la cohomologie de Hochschild de $G$ à coefficients dans $\mathbb{V}$. Une application du théorème [47, I Thm. 5.3.1] montre alors que $H^{i}(K(G, 1), V)$ se calcule aussi par foncteurs dérivés dans la catégorie $\operatorname{Rep}_{k}(G)$. Ce qu'il fallait démontrer.

Corollaire 1.5.2. Pour toute représentation linéaire $V$ d'un schéma en groupes affine $G$, on a pour tout $i \geq 0$

$$
H^{i}(K(G, 1), V) \simeq \operatorname{Colim}_{j} H^{i}\left(K(G, 1), V_{j}\right),
$$

où $V_{j}$ parcourt les sous-représentations linéaires de $V$ qui sont de dimension finie sur $k$.

Preuve: D'après le lemme précédent et [47, I Prop. 5.2.1] on sait que les groupes de cohomologie $H^{i}(K(G, 1), V)$ sont isomorphes aux groupes de cohomologie de Hochschild de $G$ à coefficients dans $\mathbb{V}$. Mais il est facile de voir que le complexe de Hochschild de $V$ est la limite inductive des complexes de Hochschild de ses sous-représentations de dimension finie. 
Rappelons qu'un schéma en groupes affine $G$ est unipotent, si pour toute représentation linéaire $V$ non-nulle on a $V^{G} \neq 0$. D'après $[11, I V, \S 2$, Prop.2.5] un schéma en groupe affine est unipotent si et seulement si pour tout sous-schéma en groupe fermé $K \hookrightarrow G$ il existe un morphisme non nul de schémas en groupes $K \longrightarrow \mathbb{G}_{a}$. De plus, la sous-catégorie pleine de $G p(k)$ formée des schémas en groupes affines unipotents est stable par noyaux, co-noyaux, images, extensions et U-limites projectives (voir [11,VI, $\S 2$, Prop.2.3]). Comme pour le cas des schémas en groupes affines, tout schéma en groupes affine unipotent est la limite projective de ses quotients de type fini sur $k$. En particulier, la catégorie des schémas en groupes affines unipotents est donc équivalente à la catégorie des Pro-objets dans la catégorie des schémas en groupes affines unipotents et de type fini sur $k$.

Notons que tout schéma en groupes affine $G$ possède un quotient unipotent maximal $G \longrightarrow G^{\text {uni }}$. Par définition, $G^{\text {uni }}$ est la limite projective de tous les quotients de $G$ qui sont des schémas en groupes affines unipotents et de type fini sur $k$. On vérifie alors que le morphisme $G \longrightarrow G^{\text {uni }}$ est universel pour les morphismes vers des schémas en groupes affines et unipotents. Le foncteur induit sur les catégories de représentations $\operatorname{Rep}_{k}\left(G^{u n i}\right) \longrightarrow \operatorname{Rep}_{k}(G)$ est exact et pleinement fidèle, et identifie $\operatorname{Rep}_{k}\left(G^{u n i}\right)$ à la sous-catégorie pleine de $\operatorname{Rep}_{k}(G)$ formée des repésentations unipotentes (i.e. colimites filtrantes d'extensions succéssives de représentations triviales).

Définition 1.5.3. Soit $G$ un $\mathbb{U}$-groupe, considéré comme un préfaisceau constant sur $(A f f / k)_{f p q c}$. Une complétion affine (resp. unipotente) de $G$ est un schéma en groupes affine $\mathcal{A}(G)$ (resp. un schéma en groupes affine et unipotent $\mathcal{U}(G)$ ), muni d'un morphisme de faisceaux en groupes $G \longrightarrow \mathcal{A}(G)$ (resp. $G \longrightarrow \mathcal{U}(G)$ ) dans $G p(k)$, qui est universel pour les morphismes vers les schémas en groupes affines (resp. les schémas en groupes affines unipotents).

Le lemme suivant est un fait très bien connu (voir [25]).

Lemme 1.5.4. Tout $\mathbb{U}$-groupe $G$ admet une complétion affine (resp. une complétion unipotente).

Preuve: Traitons le cas des complétions affines, celui des complétions unipotentes et en tout point similaire. Pour les besoins de la démonstration, rappelons que pour tout objet $X \in C$ dans une $\mathbb{V}$-catégorie et toute sous-catégorie pleine $\mathbb{V}$-petite $D \subset C$, on peut former la catégorie $\mathbb{V}$-petite $X / D$, des objets de $D$ sous $X$. Ses objets sont les paires $(Y, u)$, où $Y$ est un objet de $D$ et $u: X \longrightarrow Y$ est un morphisme dans $C$. Un morphisme dans $X / D$ de $(Y, u)$ vers $(Z, v)$ est la donnée d'un morphisme $f: Y \longrightarrow Z$ dans $D$ tel que $f \circ u=v$.

Notons $G p A l g$ la sous-catégorie pleine de $G p(k)$ formée des schémas en groupes affines et de type fini sur $k$. Posons

$$
G \longrightarrow \mathcal{A}(G):=\operatorname{Lim}_{H \in G / G p A l g} H
$$

où la limite est prise dans la catégorie des faisceaux en $\mathbb{V}$-groupes $G p(k)$. Comme la catégorie $G p A l g$ consiste en des schémas de types finis sur $k$, elle possède une 
sous-catégorie pleine $\mathbb{U}$-petite qui lui est équivalente. Ainsi, $G$ étant un élément de $\mathbb{U}$, la catégorie $G / G p A l g$ est en réalité équivalente à une catégorie U-petite. Ceci implique que $\mathcal{A}(G)$ est isomorphe à une U-limite projective de schémas en groupes affines et est donc lui-même un schéma un groupes affine. De plus, il est clair par construction que tous les morphismes vers un schéma en groupes affine de type fini $G \longrightarrow H$ se factorisent par le morphisme naturel $G \longrightarrow \mathcal{A}(G)$. Mais comme tout schéma en groupes affine est une $\mathbb{U}$-limite projective de schémas en groupes affines et de type fini, on voit que $G \longrightarrow \mathcal{A}(G)$ possède la propriété universelle requise.

Remarquons que d'après les propriétés universelles, pour tout $\mathbb{U}$-groupe $\Gamma$ on a un isomorphisme $\mathcal{U}(\Gamma) \simeq \mathcal{A}(\Gamma)^{\text {uni }}$.

\section{Champs affines}

Nous présentons dans ce chapitre la première des deux notions fondamentales de cet article, celle de champ affine. Il s'agit d'une version de nature homotopique de la notion de schéma affine, qui est définie directement à partir d'une structure de catégorie de modèles simpliciale sur la catégorie des $k$-algèbres co-simpliciales. Cette catégorie de modèles sera alors utilisée pour définir le foncteur dérivé du foncteur qui à une $k$-algèbre $A$ associe le schéma affine $\operatorname{Spec} A$. Nous montrerons que ce foncteur dérivé induit un foncteur pleinement fidèle de la catégorie homotopique des $k$-algèbres co-simpliciales dans la catégorie homotopique des préfaisceaux simpliciaux sur $(A f f / k)_{f p q c}$. La catégorie des champs affines sera alors définie comme la sous-catégorie image essentielle de ce foncteur. Ainsi, tout comme la catégorie des schémas affines est équivalente à la catégorie (opposée) des $k$-algèbres, la catégorie des champs affines sera équivalente à la catégorie homotopique (opposée) des $k$-algèbres co-simpliciales.

Par la suite, nous définirons la notion d'affination d'un préfaisceau simplicial, et nous en donnerons un critère d'existence. En particulier, nous montrerons que tout ensemble simplicial appartenant à $\mathbb{U}$ possède une affination, dont nous donnerons une description conjecturale des faisceaux d'homotopie pour des ensembles simpliciaux simplement connexes et de type fini.

Enfin, lorsque l'anneau de base est un corps, nous démontrerons un critère pour caractériser les champs affines et connexes à l'aide de leurs faisceaux d'homotopie. Nous en déduirons les analogues des théorèmes standards de l'homotopie rationnelle et $p$-adique, montrant ainsi que la théorie des champs affines est un cadre adéquat pour l'étude des modèles algébriques des types d'homotopie.

Pour tout ce chapitre $k$ sera un anneau commutatif unitaire appartenant à $\mathbb{U}$, et $(A f f / k)_{f p q c}$ le site des $\mathbb{U}$-schémas affines sur Speck muni de la topologie fidèlement plate et quasi-compacte (voir [47, VI.6.3]). Nous noterons $S \operatorname{Pr}(k)$ 
la catégorie des préfaisceaux en $\mathbb{V}$-ensembles simpliciaux sur $(A f f / k)_{f p q c}$, munie de sa structure de catégorie de modèles décrite dans le chapitre précédent, et $\operatorname{Ho}(S \operatorname{Pr}(k))$ sa catégorie homotopique. Nous verrons systématiquement les $\mathbb{V}$ ensembles simpliciaux comme des préfaisceaux simpliciaux constants sur le site $(A f f / k)_{f p q c}$. Ceci permet de voir $\mathbb{V}-S E n s$ somme une sous-catégorie pleine de $S \operatorname{Pr}(k)$. Il faut cependant se méfier du fait que ce plongement n'induit pas un plongement au niveau des catégories homotopiques.

La catégorie des $k$-algèbres commutatives et unitaires de $\mathbb{V}$ sera notée $k-A l g$. A tout objet $A \in k-A l g$ nous associerons le préfaisceau d'ensembles sur $A f f / k$, qui à un schéma affine $S=\operatorname{Spec} B$ fait correspondre l'ensemble $\operatorname{Hom}_{k-A l g}(A, B)$. Ce préfaisceau sera noté $\operatorname{Spec} A \in S \operatorname{Pr}(k)$. Remarquer que lorsque $A$ est un élément de $\mathbb{U}$, alors $\operatorname{Spec} A$ est représentable par le schéma affine $\operatorname{Spec} A \in A f f / k$.

Par convention, nous réserverons l'expression schéma affine pour désigner les objets de $A f f / k$, ou encore les préfaisceaux qu'ils représentent. Ces seront donc toujours des U-schémas. Nous parlerons aussi de champ représentable pour désigner tout objet de $H o(S \operatorname{Pr}(k))$ isomorphe à un schéma affine (et donc de la forme $\operatorname{Spec} A$ avec $A$ appartenant à $\mathbb{U})$.

\subsection{Rappel sur les algèbres co-simpliciales}

Rappelons tout d'abord que la catégorie $k-A l g$ des $\mathbb{V}$-algèbres commutatives et unitaires sur $k$ possède tout type de limites et de colimites paramétrées par des ensembles de $\mathbb{V}$. Les limites se calculent dans la catégorie des $k$-modules et les sommes et coproduits finis sont donnés par les produits tensoriels. Comme il est expliqué dans [15, II Ex. 2.8], la catégorie $k-A l g^{\Delta}$, des objets co-simpliciaux dans $k-A l g$, est alors munie d'une structure de catégorie simpliciale. Rappelons que pour $X$ un $\mathbb{V}$-ensemble simplicial et $A \in k-A l g^{\Delta}$ l'objet $A^{X}$ est défini par

$$
\begin{aligned}
& A^{X}: \Delta \longrightarrow k-A l g \\
& {[n] \mapsto \prod_{X_{n}} A_{n} .}
\end{aligned}
$$

On définit alors l'ensemble simplicial des morphismes entre deux objets $A, B \in$ $k-A l g^{\Delta}$ par la formule usuelle

$$
\begin{array}{cccc}
\underline{\operatorname{Hom}}_{k-a l g}(A, B): & \Delta^{o} & \longrightarrow & \mathbb{V}-S E n s \\
& {[n]} & \mapsto & \operatorname{Hom}_{k-\operatorname{Alg}^{\Delta}}\left(A, B^{\Delta^{n}}\right) .
\end{array}
$$

Enfin, pour $X \in \mathbb{V}-S E n s$ et $A \in k-A l g^{\Delta}$ nous noterons $X \otimes A$ le produit externe de $A$ par $X$. On dispose des eternels isomorphismes d'adjonctions

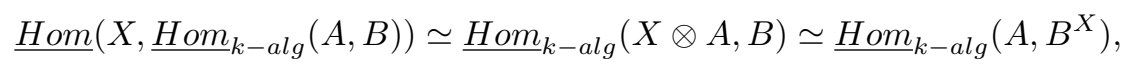

pour $X \in \mathbb{V}-S E n s$ et $A, B \in k-A l g^{\Delta}$.

Nous identifierons la catégorie $k-A l g$ à la sous-catégorie pleine des objets constants dans $k-A l g^{\Delta}$. Remarquons que pour tout $\mathbb{V}$-ensemble simplicial $X, k^{X}$ 
est la $k$-algèbre co-simpliciale de cohomologie de $k$. Explicitement on a

$$
\begin{array}{ccc}
k^{X}: \Delta & \longrightarrow & k-A l g \\
{[n]} & \mapsto \quad \operatorname{Hom}\left(X_{n}, k\right) .
\end{array}
$$

Remarquer aussi que le foncteur $k^{(-)}: \mathbb{V}-S E n s \longrightarrow\left(k-A l g^{\Delta}\right)^{o}$ est l'adjoint à gauche du foncteur $\underline{\operatorname{Hom}}_{k-\text { alg }}(-, k): k-A l g^{\Delta} \longrightarrow \mathbb{V}-$ SEns.

Notons $C_{+}(k)$ la catégorie des complexes de co-chaines concentrés en degrés positifs et appartenant à $\mathbb{V}$, et $k-\operatorname{Mod}^{\Delta}$ la catégorie des $k$-modules co-simpliciaux de $\mathbb{V}$. Il existe alors deux foncteurs inverses l'un de l'autres (c'est la correspondance duale de Dold-Puppe, voir [34, 1.4])

$$
N: k-\operatorname{Mod}^{\Delta} \longrightarrow C_{+} \quad \Gamma: C_{+} \longrightarrow k-\operatorname{Mod}^{\Delta},
$$

où pour $M \in k-\operatorname{Mod}^{\Delta}, N(M)$ est le complexe des co-chaines normalisées de $M$. Par définition, les $k$-modules de cohomologie d'un objet $M \in k-\operatorname{Mod}^{\Delta}$ seront définis par

$$
H^{i}(M):=H^{i}(N(M)) .
$$

En transportant la structure de modèle de $C_{+}$à $k-M_{o d}{ }^{\Delta}$ on déduit une structure de catégorie de modèles sur les $k$-modules co-simpliciaux pour laquelle les équivalences sont les quasi-isomorphismes (i.e. morphismes induisant des isomorphismes sur tous les $H^{i}$ ), et les fibrations sont les épimorphismes.

Le produit tensoriel de $k$-modules s'étend de façon naturelle en un produit tensoriel sur $k-M o d^{\Delta}$. De plus, la catégorie $k-M_{o d}{ }^{\Delta}$ est aussi munie d'une structure simpliciale, qui fait de $k-\operatorname{Mod}^{\Delta}$ une catégorie en algèbre fermée sur la catégorie monoïdale fermée $\mathbb{V}-S E n s$ (voir [26, Def.4.1.9, Def. 4.1.13]). Pour deux objets $M$ et $N$ dans $k-\operatorname{Mod}^{\Delta}$, on dispose de deux morphismes naturels, qui sont des quasi-isomorphismes inverses l'un de l'autre

$$
N(M \otimes N) \longrightarrow N(M) \otimes N(N) \longrightarrow N(M \otimes N),
$$

et qui sont de plus associatifs et unitaires (c'est le théorème d'Eilenberg-Zilber, voir [15, $\S I V$ Thm. 2.4]). Ceci permet en particulier de montrer que les foncteurs $N$ et $\Gamma$ préservent les homotopies. Ainsi, deux morphismes dans $k-\operatorname{Mod}^{\Delta}$ sont homotopes si et seulement si leur image dans $C_{+}$le sont (c'est l'énoncé dual de [12, Thm. 2.6]).

L'oubli de la loi d'algèbre définit un foncteur $k-A l g^{\Delta} \longrightarrow k-\operatorname{Mod}^{\Delta}$, qui à une $k$-algèbre co-simpliciale fait correspondre son $k$-module co-simplicial sousjacent. Par souci de légèreté nous noterons encore $A$ le $k$-module co-simplicial sous-jacent à une $k$-algèbre co-simpliciale $A$. En particulier nous utiliserons les notations $N(A), H^{*}(A) \ldots$ pour $A \in k-A l g^{\Delta}$. Notons au passage que $H^{*}(A)$ est toujours muni d'une structure de $k$-algèbre commutative graduée et unitaire induite par la structure de $k$-algèbre sur $A$.

Définition 2.1.1. Soit $f: A \longrightarrow B$ un morphisme de $k$-algèbres co-simpliciales. 
- Nous dirons que $f$ est une équivalence si pour tout entier $i$ le morphisme induit

$$
H^{i}(f): H^{i}(A) \longrightarrow H^{i}(B)
$$

est un isomorphisme de $k$-modules.

- Nous dirons que $f$ est une fibration si pour tout entier $n$ le morphisme induit

$$
f_{n}: A_{n} \longrightarrow B_{n}
$$

est un morphisme surjectif.

- Nous dirons que $f$ est une cofibration s'il possède la propriété de relèvement à gauche par rapport à toutes les fibrations triviales (voir [26, Def. 1.1.2]).

Théorème 2.1.2. Avec les définitions précédentes, la catégorie $k-A_{l} g^{\Delta}$ est une catégorie de modèles fermée simpliciale.

Esquisse de preuve: C'est un fait standard qui se démontre à l'aide de l'argument du petit objet. La preuve que nous allons esquisser ici est une adaptation immédiate de la preuve du théorème [4, Thm. 4.3]. Nous aurons besoin pour cela d'introduire les cofibrations et cofibrations triviales élémentaires suivantes.

Pour tout entier $n \geq 0$, notons $k[n]$ le complexe de co-chaine de $k$-modules égal à $k$ en degré $n$ et 0 partout ailleurs. Nous noterons aussi $k\langle n-1, n\rangle$ le complexe de co-chaine égal à $k$ en degrés $n-1$ et $n, 0$ partout ailleurs et avec comme différentielle $d^{n-1}=i d: k \rightarrow k$. Par convention $k<-1,0>=0$. Il existe des morphismes naturels de complexes $k[n] \longrightarrow k<n-1, n>$. Nous noterons pour tout $n$

$$
S_{k}^{n}:=\Gamma(k[n]) \quad D_{k}^{n}:=\Gamma(k<n-1, n>) .
$$

Ce sont des $k$-modules co-simpliciaux munis de morphismes $S_{k}^{n} \longrightarrow D_{k}^{n}$. Les $k$ algèbres commutatives unitaires co-simpliciales libres engendrées par ces $k$-modules co-simpliciaux seront notés

$$
S(n):=S^{*}\left(S_{k}^{n}\right) \in k-A l g^{\Delta} \quad D(n):=S^{*}\left(D_{k}^{n}\right) \in k-A l g^{\Delta} .
$$

Remarquer que $S(0) \simeq k[T]$, et $D(0) \simeq k$.

Nous définissons $I$ comme étant l'ensemble des morphismes naturels $S(n) \longrightarrow$ $D(n)$ pour $n \geq 0$, et des morphismes (correspondant à l'unité) $k \longrightarrow S(n)$ pour $n \geq 0$. De même, l'ensemble $J$ est l'ensemble des morphismes $k \longrightarrow D(n)$ pour $n \geq 0$.

On peut vérifier que pour tout $A \in k-A l g^{\Delta}$, la donnée d'un morphisme $x: S(n) \longrightarrow A$ est équivalente à la donnée d'un cocycle dans le complexe normalisé associé $x \in Z^{n}(N(A))$. De même, la donnée d'un morphisme $y: D(n) \longrightarrow A$ est équivalent à la donnée d'un élément $y \in N(A)_{n-1}$. Ainsi, comme un morphisme de $k-A l g^{\Delta}$ est surjectif si et seulement si le morphisme correspondant sur les complexes normalisés est surjectif, on vérifie à l'aide des propriétés universelles précédentes que les morphismes de $I$ sont des cofibrations, et que ceux de $J$ sont des cofibrations triviales. 
Pour démontrer que $k-A l g^{\Delta}$ est une catégorie de modèles fermée on utilise l'argument du petit objet relativement aux ensembles $I$ et $J$ (voir [26, 2.1.17]). Il est alors bien connu qu'il suffit de montrer les trois assertions suivantes.

1. Un morphisme $f: A \longrightarrow B$ de $k-A l g^{\Delta}$ est une fibration (resp. une fibration triviale) si et seulement s'il possède la propriété de relèvement par rapport à tous les morphismes de $J$ (resp. de $I$ ).

2. Une colimite filtrante d'équivalences dans $k-A l g^{\Delta}$ est une équivalence.

3. Pour tout morphisme $\tau_{n}: k \longrightarrow D(n)$ dans $J$, et tout objet $A \in k-A l g^{\Delta}$, le morphisme naturel $A \longrightarrow A \otimes D(n)$ est une équivalence.

La propriété (1) se déduit immédiatement des propriétés universelles des objets $S(n)$ et $D(n)$ et des définitions des fibrations et des équivalences. La propriété (2) est vraie car les foncteurs $H^{i}: k-A l g^{\Delta} \longrightarrow k-\operatorname{Mod}$ commutent avec les colimites filtrantes.

Pour démontrer $(3)$ on commence par remarquer que les morphismes $0 \longrightarrow$ $k\langle n-1, n\rangle$ sont des équivalences d'homotopie. Comme le foncteur $\Gamma$ préserve les homotopies on trouve que $0 \longrightarrow D_{k}^{n}$ est aussi une équivalence par homotopie dans $k-M_{o d}{ }^{\Delta}$. De plus, le foncteur qui à $M \in k-\operatorname{Mod}^{\Delta}$ associe $S^{*}(M) \in$ $k-A l g^{\Delta}$ est obtenu par prolongation du foncteur $S^{*}: k-\operatorname{Mod} \longrightarrow k-A l g$, et d'après l'énoncé dual de [12, Thm. 5.6] il préserve donc les homotopies. Ainsi, on trouve que les morphismes $k \longrightarrow D(n)$ sont des équivalences d'homotopie. Soit $h: D(n) \longrightarrow D(n)^{\Delta^{1}}$ une contraction de $D(n)$ sur $k$. En tensorisant par $A$ on trouve un nouveau morphisme

$$
h: A \otimes D(n) \longrightarrow A \otimes\left(D(n)^{\Delta^{1}}\right) \simeq(A \otimes D(n))^{\Delta^{1}},
$$

qui est un contraction de $A \otimes D(n)$ sur $A$. Ainsi, le morphisme $A \longrightarrow A \otimes D(n)$ est une équivalence d'homotopie et donc une équivalence.

Il nous reste à démontrer l'axiome SM7 de [15, §II Def. 3.1]. Pour cela, il faut montrer que pour toute cofibration d'ensembles simpliciaux $u: X \longrightarrow Y$, et toute cofibration $v: A \longrightarrow B$ dans $k-A l g$, le morphisme naturel

$$
A^{X} \longrightarrow B^{X} \prod_{B^{Y}} A^{Y}
$$

est une fibration, qui est une équivalence dès que $u$ ou $v$ en est une. Le fait que c'est une fibration est clair. De plus, il est immédiat de voir que le morphisme $B^{X} \longrightarrow$ $B^{Y}$ est une fibration, et donc que le morphisme de complexes $N\left(B^{X}\right) \longrightarrow N\left(B^{Y}\right)$ est surjectif. Comme $N$ commute avec les produits fibrés, on dispose ainsi d'une suite exacte longue de Mayer-Vietoris

$$
\begin{gathered}
0 \longrightarrow H^{0}\left(B^{X} \prod_{B^{Y}} A^{Y}\right) \longrightarrow H^{0}\left(B^{X}\right) \prod H^{0}\left(A^{Y}\right) \longrightarrow H^{0}\left(B^{Y}\right) \longrightarrow H^{i}\left(B^{X} \prod_{B^{Y}} A^{Y}\right) \longrightarrow H^{i}\left(B^{X}\right) \prod H^{i}\left(A^{Y}\right) \longrightarrow \\
\cdots \longrightarrow \\
H^{i+1}\left(B^{X} \prod_{B^{Y}} A^{Y}\right) \longrightarrow \cdots
\end{gathered}
$$


On voit donc qu'il suffit de montrer que le morphisme $A^{X} \longrightarrow B^{X}$ (resp. $A^{X} \longrightarrow$ $A^{Y}$ ) est une équivalence lorsque $u$ (resp. $v$ ) en est une. Mais ceci se ramène à l'énoncé connu que le foncteur qui à un bi-complexe positif associe son complexe total préserve les quasi-isomorphismes.

Remarque: Remarquer que tous les objets de $k-A l g^{\Delta}$ sont fibrants. De plus, si $A$ est une $k$-algèbre co-simpliciale constante, alors $A$ est un objet cofibrant dans $k-A l g^{\Delta}$. En effet, la donnée d'un morphisme $A \longrightarrow B$ dans $k-A l g^{\Delta}$ est équivalente à la donnée d'un morphisme de $k$-algèbres $A \longrightarrow H^{0}(B)$. Le morphisme unité $k \longrightarrow A$ possède donc la propriété de relèvement par rapport à toutes les fibrations triviales.

Comme la catégorie de modèles $k-A l g^{\Delta}$ est simpliciale, on peut définir des Hom simpliciaux en un sens dérivés (voir [26, Thm. 4.3.2]). Ils seront notés $\mathbb{R}_{\underline{H o m}_{k-a l g}}$. Ainsi, pour $A$ et $B$ deux objets de $k-A l g^{\Delta}, \mathbb{R} \underline{H o m}_{k-a l g}(A, B)$ est un objet bien défini dans $H o(\mathbb{V}-S E n s)$ et fonctoriel en $A$ et $B$. Cela munit la catégorie homotopique $H o\left(k-A l g^{\Delta}\right)$ d'une structure de catégorie enrichie dans $H o(\mathbb{V}-S E n s)$.

Nous noterons aussi comme il en est l'usage $[-,-]_{k-a l g}$ les ensembles de morphismes dans la catégorie homotopique $H o\left(k-A l g^{\Delta}\right)$. Remarquons au passage qu'il existe des isomorphismes naturels pour tout $A, B \in k-A l g^{\Delta}$

$$
[A, B]_{k-a l g} \simeq \pi_{0} \underline{\mathbb{H o m}}_{k-\text { alg }}(A, B)
$$

La structure simpliciale sur la catégorie de modèles $k-A l g^{\Delta}$ nous permettra par la suite de parler de limites et colimites homotopiques, qui sont définies de façon standard (voir $[22, \S 19]$ ). Nous utiliserons souvent que toute $k$-algèbre cosimpliciale $A$ est équivalente à la limite homotopique $\operatorname{Holim}_{[n] \in \Delta} A_{n}$, où chaque $A_{n}$ est vu comme une $k$-algèbre co-simpliciale constante (ce qui se vérifie immédiatement en utilisant que tout ensemble simplicial $X$ est équivalent à la colimite homotopique Hocolim $\left._{[n] \in \Delta^{\circ}} X_{n}\right)$.

Pour clôre ce paragraphe signalons que nous aurons a utiliser la sous-catégorie pleine de $k-A l g^{\Delta}$ formée des $k$-algèbres co-simpliciales appartenant à $\mathbb{U}$. Cette sous-catégorie possède de très nombreuses propriétés de stabilité. Nous utiliserons en particulier que pour tout morphisme $f: A \longrightarrow B$ de $k$-algèbres co-simpliciales de $\mathbb{U}$, les factorisations fonctorielles définies à l'aide des ensembles générateurs $I$ et $J$ des cofibrations et des cofibrations triviales, appartiennent encore à $\mathbb{U}$. En particulier, toute $k$-algèbre co-simpliciale de $\mathbb{U}$ possède un modèle cofibrant qui appartient encore à $\mathbb{U}$. 


\subsection{Champs affines}

Pour $A \in k-A l g^{\Delta}$ une $k$-algèbre co-simpliciale, on peut considérer le préfaisceau simplicial $\operatorname{Spec} A \in S \operatorname{Pr}(k)$ défini par

$$
\begin{aligned}
& \text { Spec } A: \quad(A f f / k)^{\circ} \longrightarrow \mathbb{V}-\text { SEns } \\
& \operatorname{Spec} B \quad \mapsto \quad \underline{H o m}_{k-a l g}(A, B) \text {. }
\end{aligned}
$$

Le préfaisceau des $n$-simplexes $\operatorname{de} \operatorname{Spec} A$ est donc isomorphe au préfaisceau représenté par le schéma affine $\operatorname{Spec} A_{n}$. Ceci définit évidemment un foncteur

$$
\text { Spec }:\left(k-A l g^{\Delta}\right)^{o} \longrightarrow S \operatorname{Pr}(k) .
$$

Ce foncteur possède un adjoint à gauche

$$
\mathcal{O}: \operatorname{SPr}(k) \longrightarrow\left(k-A l g^{\Delta}\right)^{o}
$$

qui est défini de la façon suivante. Pour tout préfaisceau simplicial $F \in \operatorname{SPr}(k)$, on définit une $k$-algèbre co-simpliciale par

$$
\mathcal{O}(F): \begin{array}{ccc}
\Delta & \longrightarrow & k-A l g \\
{[n]} & \mapsto \quad \operatorname{Hom}\left(F_{n}, \mathcal{O}\right) .
\end{array}
$$

Dans cette définition $\mathcal{O}$ désigne aussi le préfaisceau en $k$-algèbres tautologique sur Aff $/ k$, qui au schéma affine $\operatorname{Spec} A$ associe $A$. Le loi de $k$-algèbre sur $\operatorname{Hom}\left(F_{n}, \mathcal{O}\right)$ est alors induite naturellement par la loi de $k$-algèbre sur le préfaisceau $\mathcal{O}$. Remarquer que $\mathcal{O}(F)$ n'est généralement pas un élément de $\mathbb{U}$.

A l'aide de ces définitions on vérifie qu'il existe des isomorphismes naturels, décrivant le comportement des foncteurs $S p e c$ et $\mathcal{O}$ relativement à la structure simpliciale,

$$
\operatorname{Spec}(X \otimes A) \simeq \operatorname{Spec}(A)^{X} \quad \mathcal{O}(X \otimes F) \simeq \mathcal{O}(F)^{X},
$$

pout tout $X \in \mathbb{V}-S E n s, A \in k-A l g^{\Delta}$ et $F \in S \operatorname{Pr}(k)$. En particulier, l'adjonction entre $S p e c$ et $\mathcal{O}$ s'étend en une adjonction simpliciale

$$
\underline{\operatorname{Hom}}_{k-a l g}(A, \mathcal{O}(F)) \simeq \underline{\operatorname{Hom}}(F, \operatorname{Spec} A) .
$$

Le lemme suivant est une remarque clé que nous utiliserons implicitement par la suite.

Lemme 2.2.1. Si $A \in k-A l g^{\Delta}$ appartient à $\mathbb{U}$ alors le morphisme d'adjonction $A \longrightarrow \mathcal{O}$ Spec $A$ est un isomorphisme.

Preuve: Le préfaisceau $\mathcal{O}$ étant représenté par le schéma affine $\operatorname{Spec} k[T]$, c'est une application du lemme de Yoneda.

Pour la proposition suivante, nous rappelons que $\operatorname{SPr}(k)$ est munie da la structure de catégorie de modèles décrite dans le chapitre précédent, et que ses équivalences sont définies relativement à la topologie fidèlement plate et quasicompacte. De même, $H o(S \operatorname{Pr}(k))$ désignera la catégorie homotopique des préfaisceaux simpliciaux sur le site $(A f f / k)_{f p q c}$. 
Proposition 2.2.2. Le foncteur Spec : $\left(k-A l g^{\Delta}\right)^{o} \longrightarrow S \operatorname{Pr}(k)$ est de Quillen à droite.

Preuve: L'axiome SM7 des catégories de modèles simpliciales implique que le foncteur $S p e c$ est de Quillen à droite lorsque $\operatorname{SPr}(k)$ est munie de sa structure forte. Par la propriété universelle des localisations de Bousfied à gauche il faut donc montrer que pour tout objet cofibrant $A \in k-A l g^{\Delta}$, le préfaisceau simplicial Spec $A$ est fibrant (voir $[22,3.4 .20]$ ). Pour cela nous allons utiliser le critère 1.1.2.

Soit $A \in k-A l g^{\Delta}$ un objet cofibrant, et $\operatorname{Spec} B_{*} \longrightarrow S p e c C$ un hyperrecouvrement dans $(A f f / k)_{f p q c}$. Ce morphisme est donc donné par un morphisme d'algèbres co-simpliciales

$$
C \longrightarrow B_{*} \text {. }
$$

Il s'agit alors de montrer que le morphisme naturel

$$
\underline{\operatorname{Hom}}_{k-a l g}(A, C) \longrightarrow \operatorname{Holim}_{[n] \in \Delta} \underline{\operatorname{Hom}}_{k-a l g}\left(A, B_{n}\right)
$$

est une équivalence. Or, comme tous les objets de $k-A l g^{\Delta}$ sont fibrants et que $A$ est cofibrant, le morphisme ci-dessus est isomorphe dans la catégorie homotopique $H o(\mathbb{V}-S E n s)$ au morphisme

$$
\mathbb{R}_{\underline{H o m}_{k-\text { alg }}}(A, C) \longrightarrow \operatorname{Holim}_{[n] \in \Delta} \mathbb{R} \underline{\operatorname{Hom}}_{k-\text { alg }}\left(A, B_{n}\right) \simeq \underline{\mathbb{R o m}}_{k-\text { alg }}\left(A, B_{*}\right) \text {. }
$$

En effet, en tant qu'objet de $k-A_{l} g^{\Delta}, \operatorname{Holim}_{[n] \in \Delta} B_{n}$ est naturellement équivalent à $B_{*}$. Il suffit ainsi de montrer que le morphisme naturel

$$
C \longrightarrow B_{*}
$$

est une équivalence dans $k-A l g^{\Delta}$. Mais en passant aux complexes normalisés associés on voit facilement que cette dernière assertion se démontre en appliquant la descente cohomologique fildèlement plate (voir $\left[49, \mathrm{Ex} . V^{b} i s\right]$ ) à l'hyperrecouvrement $\operatorname{Spec} B_{*} \longrightarrow S p e c C$, et au complexe de faisceaux quasi-cohérents concentré en degré zéro $\mathcal{O}_{S p e c} C$.

Corollaire 2.2.3. Pour toute k-algèbre co-simpliciale $A$ de $\mathbb{U}$, le morphisme d'adjonction

$$
A \longrightarrow \mathbb{L} \mathcal{O} \mathbb{R} \operatorname{Spec} A
$$

est un isomorphisme dans $\mathrm{Ho}\left(k-\mathrm{Alg}^{\Delta}\right)$.

En particulier, le foncteur dérivé

$$
\mathbb{R} \text { Spec }: H o\left(k-A l g^{\Delta}\right) \longrightarrow H o(\operatorname{SPr}(k))
$$

restreint aux $k$-algèbres co-simpliciales isomorphes dans $H o\left(k-A l g^{\Delta}\right)$ à des objets de $\mathbb{U}$, est un foncteur pleinement fidèle.

Preuve: Il suffit de montrer que pour tout $k$-algèbre co-simpliciale $A$ de $\mathbb{U}$, qui est un objet cofibrant dans $k-A l g^{\Delta}$, le morphisme naturel $A \longrightarrow \mathbb{L O S p e c} A$ est un isomorphisme. Considèrons l'isomorphisme naturel dans $\operatorname{Ho}(\operatorname{SPr}(k))$

$$
\operatorname{Spec} A \simeq \operatorname{Hocolim}_{[n] \in \Delta^{\circ}} \operatorname{Spec} A_{n} .
$$


Remarquons que comme toute les $k$-algèbres $A_{n}$ sont des éléments de $\mathbb{U}$, le préfaisceau simplicial représenté par $S p e c A_{n}$ est un objet cofibrant dans $\operatorname{SPr}(k)$. Ainsi, comme $\mathcal{O}$ est de Quillen à gauche et que $A_{n}$ est un objet cofibrant dans $k-A l g^{\Delta}$, le lemme 2.2.1 implique que l'on a $A_{n} \simeq \mathbb{L O}\left(\mathbb{R} S\right.$ pec $\left.A_{n}\right)$. Le morphisme d'adjonction s'écrit donc

$$
\begin{aligned}
& A \simeq \text { Holim }_{[n] \in \Delta} A_{n} \simeq \operatorname{Holim}_{[n] \in \Delta} \mathbb{L} \mathcal{O}\left(\mathbb{R} \text { Spec } A_{n}\right) \\
& \simeq \mathbb{L} \mathcal{O}\left(\text { Hocolim }_{[n] \in \Delta} \mathbb{R} \text { Spec } A_{n}\right) \simeq \mathbb{L} \mathcal{O}(\text { Spec } A) .
\end{aligned}
$$

Il s'agit donc bien d'un isomorphisme.

Définition 2.2.4. Un champ affine (sur $k$ ) est un champ $F \in S \operatorname{Pr}(k)$, qui vu comme un objet de $H o(S \operatorname{Pr}(k))$ est isomorphe à $\mathbb{R} S$ pec $A$, pour $A$ une $k$-algèbre co-simpliciale appartenant à $\mathbb{U}$. Les morphismes de champs affines sont simplement les morphismes de champs.

Par abus de langage, nous dirons qu'un préfaisceau simplicial $F$ est un champ affine, si un modèle fibrant de $F$ est un champ affine.

\section{Remarques:}

- Il est important de noter que $\mathbb{R} S p e c A$ est par définition le préfaisceau simplicial Spec $Q A$, où $Q A \longrightarrow A$ est un remplacement cofibrant de $A$ dans $k-A l g^{\Delta}$. Ainsi, un préfaisceau de la forme $\operatorname{Spec} B$, où $B$ est une $k$-algèbre co-simpliciale de $\mathbb{U}$ quelconque n'est à prioris pas un champ affine. Comme le montre le théorème 2.2.9 et le contre-exemple qui suit le corollaire 2.2.11 la notion de champ affine est en réalité beaucoup plus forte que celle d'être équivalent à un schéma simplicial affine de $\mathbb{U}$.

- Noter qu'un préfaisceau simplicial de la forme $\operatorname{Spec} A$, où $A$ est une $k$-algèbre co-simpliciale de $\mathbb{V}$ qui n'est pas équivalente dans $k-A l g^{\Delta}$ à un élément de $\mathbb{U}$, n'est pas considéré comme un champ affine. En contre partie tous les préfaisceaux représentables par des objets de $A f f / k$ le sont. En effet, pour toute $k$-algèbre $A$ de $\mathbb{U}$, la $k$-algèbre co-simplciale constante de valeurs $A$ appartient à $\mathbb{U}$ et est un objet cofibrant. Ainsi, $\mathbb{R} S p e c A$ est isomorphe dans $H o(S \operatorname{Pr}(k))$ au préfaisceau représenté par le schéma affine $\operatorname{Spec} A$.

- D'un point de vue terminologique il est important de ne pas confondre champs affines et schémas affines. Tout schéma affine est un champ affine, mais il existe des champs affines et 0 -tronqués (i.e. des faisceaux qui sont affines en tant que champs) qui ne sont pas représentables par des schémas affines (un tel exemple est donné à la suite du corollaire 2.2.10). Il est donc parfois préférable d'utiliser l'expression champs représentables pour signifier schémas affines et éviter ainsi des confusions.

Les premiers exemples de champs affines sont les champs classifiants $K\left(\mathbb{G}_{a}, i\right)$, où $\mathbb{G}_{a}$ est le groupe additif. Ce sont en réalité les pièces élémentaires de la théorie, et nous verrons que tout champ affine est une certaine limite homotopique construite à partir de tels champs. 
Lemme 2.2.5. Notons $\mathbb{G}_{a}$ le préfaisceau en groupes additifs sous-jacent au préfaisceaux en $k$-algèbres $\mathcal{O}$, et $S(i)$ la $k$-algèbre co-simpliciale définie lors de la preuve $d u$ théorème 2.1.2. Alors, pour tout entier $i$, le préfaisceau simplicial $K\left(\mathbb{G}_{a}, i\right) \in$ $S \operatorname{Pr}(k)$ est un champ affine, équivalent pour la structure forte à Spec $S(i)$.

Preuve: A l'aide de la propriété universelle satisfaite par $S(i)$ on voit facilement que pour tout $\operatorname{Spec} B \in A f f / k, \pi_{m}^{p r}(\operatorname{Spec}(S(i))(B))=0$ pour $m \neq i$, et $\pi_{i}^{p r}(\operatorname{Spec}(S(i))(B)) \simeq B$. De plus, le préfaisceau des 0-simplexes de $\operatorname{Spec} S(i)$ est isomorphe au préfaisceau constant $*$. C'est alors un fait standard que tout préfaisceau simplicial $F$, tel que $F_{0}=*$, et $\pi_{m}^{p r}(F, *)=0$ pour $m \neq i$ est équivalent pour la structure forte sur $\operatorname{SPr}(k)$ au préfaisceau $K\left(\pi_{i}^{p r}(F, *), i\right)$. On a donc une équivalence forte $\operatorname{Spec} S(i) \simeq K\left(\mathbb{G}_{a}, i\right)$.

Corollaire 2.2.6. Pour tout préfaisceau simplicial $F \in S \operatorname{Pr}(k)$ on a des isomorphismes fonctoriels

$$
H^{i}\left(F, \mathbb{G}_{a}\right) \simeq H^{i}(\mathbb{L} \mathcal{O}(F)) .
$$

En particulier, pour tout $k$-algèbre co-simpliciale $A$ de $\mathbb{U}$, il existe des isomorphismes fonctoriels

$$
H^{i}(A) \simeq H^{i}\left(\mathbb{R} \operatorname{Spec} A, \mathbb{G}_{a}\right) .
$$

Preuve: Le membre de droite est par définition isomorphe à l'ensemble des morphismes $\left[F, K\left(\mathbb{G}_{a}, i\right)\right]_{S \operatorname{Pr}(k)}$. Ainsi, en utilisant l'adjonction $(\mathcal{O}, S p e c)$ et la propriété universelle satifaite par $S(i)$, on trouve des isomorphismes naturels

$$
H^{i}\left(F, \mathbb{G}_{a}\right) \simeq\left[F, K\left(\mathbb{G}_{a}, i\right)\right]_{S P r(k)} \simeq[S(i), \mathbb{L} \mathcal{O}(F)]_{k-a l g} \simeq H^{i}(\mathbb{L} \mathcal{O}(F)) .
$$

La seconde assertion se déduit de la première et du corollaire 2.2.3.

Proposition 2.2.7. Les champs affines sont stables par U-limites homotopiques.

Preuve: Comme toute U-limite homotopique se décompose en une composition de produits homotopiques, de produits fibrés homotopiques et de limites homotopiques suivant la catégorie $\mathbb{N}: \cdots \rightarrow n \rightarrow n-1 \rightarrow \cdots \rightarrow 1$, il suffit de vérifier que les champs affines sont stables par ces trois types de limites homotopiques.

Si $I \in \mathbb{U}$, et $F_{i} \in H o(S \operatorname{Pr}(k))$ sont des champs affines où $i$ parcourt $I$, on peut écrire $F_{i} \simeq \operatorname{Spec} A_{i}$, avec $A_{i}$ une $k$-algèbre co-simpliciale cofibrante appartenant à $\mathbb{U}$. Dans ce cas, comme Spec est un foncteur de Quillen à droite, on a

$$
\mathbb{R} \prod_{i \in I} F_{i} \simeq \operatorname{Spec}\left(\coprod_{i \in I} A_{i}\right)
$$

ce qui montre que $\mathbb{R} \prod_{i \in I} F_{i}$ est bien un champ affine.

Soit $F_{1} \stackrel{u}{\longrightarrow} F_{0} \stackrel{v}{\longleftarrow} F_{2}$ un diagramme de champs affines dans $\operatorname{SPr}(k)$. A équivalence près, on peut d'après le corollaire 2.2.3 représenter ce diagramme 
comme l'image d'un diagramme de $k$-algèbres co-simpliciales cofibrantes et appartenant à $\mathbb{U}, A_{1} \ll^{u} A_{0} \underset{v}{\longrightarrow} A_{2}$, avec $u$ et $v$ des cofibrations. On a alors

$$
F_{1} \times F_{F_{0}}^{h} F_{2} \simeq \operatorname{Spec}\left(A_{1} \coprod_{A_{0}} A_{2}\right),
$$

ce qui implique que le produit fibré homotopique $F_{1} \times_{F_{0}}^{h} F_{2}$ est un champ affine.

Enfin, soit $\ldots F_{n} \stackrel{u_{n}}{\longrightarrow} F_{n-1} \stackrel{u_{n-1}}{\longrightarrow} \cdots \stackrel{u_{2}}{\longrightarrow} F_{1}$ un foncteur de $\mathbb{N}$ vers la catégorie $\operatorname{SPr}(k)$ tel que chaque $F_{n}$ soit un champ affine. Par un argument de récurence sur $n$, on voit que le peut remplacer ce diagramme par un diagramme équivalent qui est image par Spec d'une suite de cofibrations de $k$-algèbres cosimpliciales cofibrantes et appartenant à $\mathbb{U}$

$$
\ldots A_{n} \stackrel{u_{n}}{\longleftarrow} A_{n-1} \stackrel{u_{n-1}}{\longleftarrow} \ldots \ll \stackrel{u_{2}}{\longleftarrow} A_{1} \text {. }
$$

Ainsi, Spec étant un foncteur de Quillen à droite on trouve

$$
\operatorname{Holim}_{n} F_{n} \simeq \operatorname{Holim}_{n} \operatorname{Spec} A_{n} \simeq \operatorname{Spec}\left(\operatorname{Colim}_{n} A_{n}\right) .
$$

Ceci montre que $\operatorname{Holim}_{n} F_{n}$ est un champ affine.

Nous sommes maintenant en mesure de donner une caractérisation des champs affines, qui à l'aide de la proposition pécédente permettra de construire de nombreux exemples. Pour cela nous aurons besoin des définitions suivantes. Le lecteur pourra trouver des détails sur la notion d'objet locaux et de localisation dans $[22]$.

Définition 2.2.8. - Un préfaisceau simplicial $F \in S \operatorname{Pr}(k)$ est sous-affine s'il existe un objet simplicial $X$ de $A f f / k$, tel que $F$ soit isomorphe dans la catégorie $H o(S \operatorname{Pr}(k))$ à $h_{X}$, le préfaisceau simplicial représenté par $X$.

- Un morphisme $f: G \longrightarrow H$ dans $\operatorname{SPr}(k)$ est une $\mathcal{O}$-équivalence si pour tout $i \geq 0$ le morphisme induit

$$
f^{*}: H^{i}\left(H, \mathbb{G}_{a}\right) \longrightarrow H^{i}\left(G, \mathbb{G}_{a}\right)
$$

est un isomorphisme.

- Un préfaisceau simplicial $F \in S \operatorname{Pr}(k)$ est $\mathcal{O}$-local si pour toute $\mathcal{O}$-équivalence $f: G \longrightarrow H$, le morphisme induit

$$
f^{*}: \mathbb{R} \underline{\operatorname{Hom}}(H, F) \longrightarrow \mathbb{R} \underline{\operatorname{Hom}}(G, F)
$$

est un isomorphisme dans $H o(\mathbb{V}-S E n s)$.

Par la suite, nous utiliserons très souvent le fait élémentaire qu'une $\mathbb{V}$-limite homotopique d'objets $\mathcal{O}$-locaux est encore un objet $\mathcal{O}$-local.

Théorème 2.2.9. Un champ $F$ est affine si et seulement s'il est sous-affine et $\mathcal{O}$ local. 
Preuve: Commençons par la necessité. Si $F$ est un champ affine on peut trouver une $k$-algèbre co-simpliciale $A$ dans $\mathbb{U}$, qui est de plus cofibrante dans $k-A l g^{\Delta}$, et tel que $F$ soit équivalent à $\operatorname{Spec} A$. Or, comme chaque $A_{n}$ est une $k$ algèbre de $\mathbb{U}$, Spec $A$ est clairement isomorphe au préfaisceau simplicial représenté par le schéma affine simplicial $[n] \mapsto S p e c A_{n}$. Ceci montre que $F$ est sous-affine.

Montrons que $F$ est $\mathcal{O}$-local. Quitte à remplacer $A$ par un objet équivalent, on peut l'écrire comme une composition

$$
A^{(n)} \longrightarrow A^{(n+1)} \longrightarrow \ldots \operatorname{Colim}_{n} A^{(n)}=A,
$$

où pour chaque entier $n$ il existe un carré cocartésien

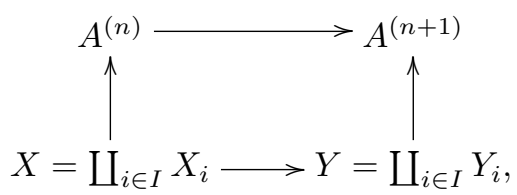

tel que chaque $X_{i} \longrightarrow Y_{i}$ soit l'une des cofibrations élémentaires décrite dans la preuve du théorème 2.1.2 (voir [26, 2.1.14]). Ainsi, comme Spec $A$ est alors équivalent à la limite homotopique $\operatorname{des} S p e c A^{(n)}$ on se ramène au cas où $A=$ $A^{(n)}$. De plus, comme $\operatorname{Spec} A^{(n)}$ est équivalent au produit fibré homotopique de $\operatorname{Spec} A^{(n-1)}$ et $\operatorname{Spec} Y$ au-dessus de $\operatorname{Spec} X$ on peut supposer par récurrence que $A=X$ ou $A=Y$. Enfin, comme $\operatorname{Spec} X$ (resp. Spec $Y$ ) est le produit homotopique $\operatorname{des} \operatorname{Spec} X_{i}$ (resp. $\operatorname{des} \operatorname{Spec} Y_{i}$ ), on peut supposer que $A$ est l'un des domaines ou l'un des codomaines des cofibrations élémentaires. Il nous suffit donc d'examiner le cas $A=S(p)$ et le cas $A=D(p)$, pour $p$ un entier positif.

Nous savons d'après le lemme 2.2.5 que Spec $S(p)$ est équivalent à $K\left(\mathbb{G}_{a}, p\right)$, qui est évidemment $\mathcal{O}$-local par définition. De même, on montre facilement à l'aide de la propriété universelle satisfaite par $D(p)$ que $\pi_{m}^{p r}(\operatorname{Spec} D(p))=*$ pour tout entier $m$, et donc que $\operatorname{Spec} D(p)$ est équivalent au préfaisceau simplicial $*$, qui est bien entendu $\mathcal{O}$-local.

Pour démontrer la suffisance, soit $F$ un préfaisceau simplicial $\mathcal{O}$-local, et considérons le morphisme d'adjonction dans $\operatorname{Ho}(\operatorname{SPr}(k))$

$$
u: F \longrightarrow \mathbb{R} \operatorname{Spec} \mathbb{L} \mathcal{O}(F) \text {. }
$$

Comme $F$ est sous-affine, l'objet $\mathbb{L} \mathcal{O}(F) \in H o\left(k-A l g^{\Delta}\right)$ est en réalité isomorphe à un objet de $\mathbb{U}$. En effet, si $X$ est un schéma affine simplicial représentant $F$, la $k$-algèbre co-simpliciale $\mathbb{L} \mathcal{O}(F)$ est équivalente à la $k$-algèbre co-simpliciale $[n] \mapsto \mathcal{O}\left(X_{n}\right)$. Ainsi, une application du corollaire 2.2.6 montre que le morphisme d'adjonction induit des isomorphismes pour tout $i$

$$
u^{*}: H^{i}\left(\mathbb{R} \operatorname{Spec} \mathbb{L} \mathcal{O}(F), \mathbb{G}_{a}\right) \simeq H^{i}(\mathbb{L} \mathcal{O}(F)) \simeq H^{i}\left(F, \mathbb{G}_{a}\right) .
$$

Ceci montre que $u$ est une $\mathcal{O}$-équivalence. Mais par hypothèse $F$ est $\mathcal{O}$-local. De même, nous avons déjà démontré que $\mathbb{R} S p e c \mathbb{L} \mathcal{O}(F)$ était $\mathcal{O}$-local. Or, il est facile de vérifier à l'aide du lemme de Yoneda qu'une $\mathcal{O}$-équivalence entre deux objets 
$\mathcal{O}$-locaux est une équivalence. Ainsi, $u$ est une équivalence, et donc $F$ est un champ affine.

\section{Remarques:}

- La preuve du théorème précédent montre en particulier que tout champ affine est construit par limite homotopique à partir de champs de la forme $K\left(\mathbb{G}_{a}, n\right)$. Ceci et la proposition 2.2.7 montrent que la catégorie des champs affines est la plus petite sous-catégorie pleine de $H o(\operatorname{SPr}(k))$ stables par U-limites homotopiques et contenant les champs $K\left(\mathbb{G}_{a}, n\right)$. C'est la raison pour laquelle les champs affines nous semblent très proches des complexes of unipotent bundles discutés dans [18, p. 446].

- Le théorème 2.2.9 montre en particulier que la notion de champ affine est intrinsèque à la théorie homotopique des préfaisceaux simpliciaux. En particulier il s'agit d'une notion qui est indépendante de la catégorie de modèles $k-A l g^{\Delta}$ (qui n'a comme rôle que de donner un modèle algébrique des champs affines). On pourrait tout aussi bien utiliser d'autres catégorie d'algèbres (comme par exemple la catégorie des $E_{\infty}$-algèbres, ou encore celle des algèbres différentielles graduées commutatives lorsque $k$ est de caractéristique nulle), la théorie des champs affines en resterait inchangée. Remarquons cependant que le corollaire 2.2.3 ne sera plus vrai pour des $E_{\infty}$-algèbre (sauf lorsque $k$ est de caractéristique nulle).

Le corollaire suivant est une extension du corollaire 2.2.3. Il montre en particulier que tout préfaisceau simplicial de taille raisonable possède une $\mathcal{O}$-localisation (i.e. un morphisme vers un objet $\mathcal{O}$-local qui est une $\mathcal{O}$-équivalence). Nous reviendrons sur cette construction lors du pargraphe suivant (voir 2.3.2).

Corollaire 2.2.10. Soit $F$ un préfaisceau simplicial tel que $\mathbb{L} \mathcal{O}(F)$ soit isomorphe dans $\mathrm{Ho}\left(k-\mathrm{Alg}^{\Delta}\right)$ à un objet de $\mathbb{U}$. Alors, le morphisme naturel $F \longrightarrow$ $\mathbb{R} S$ pec $\mathbb{L} \mathcal{O}(F)$ est universel dans la catégorie homotopique $H o(S \operatorname{Pr}(k))$ pour les morphismes de $F$ vers des objets $\mathcal{O}$-locaux (et en particulier vers des champs affines).

En particulier, si $F$ est un champ affine alors le morphisme d'adjonction $F \longrightarrow \mathbb{R} \operatorname{Spec} \mathbb{L} \mathcal{O}(F)$ est un isomorphisme dans $H o(\operatorname{SPr}(k))$.

Preuve: Le théorème 2.2.9 montre que $\mathbb{R} S p e c \mathbb{L} \mathcal{O}(F)$ est $\mathcal{O}$-local, et une application du corollaire 2.2.6 montre que le morphisme naturel $F \longrightarrow \mathbb{R} \operatorname{Spec} \mathbb{L} \mathcal{O}(F)$ est une $\mathcal{O}$-équivalence. La propriété universelle des objets $\mathcal{O}$-locaux implique alors le résultat.

\section{Exemples:}

- Voici le contre-exemple typique d'un champ sous-affine $F$ qui n'est pas affine. Soit $D$ un U-schéma en groupes diagonalisable sur $k$ (voir [47, Ex.I 4.4]), et $F=K(D, 1)$, qui est clairement sous-affine. Alors, d'après le lemme 1.5 .1 et 
$[47, E x . I$ 5.3.3] on voit que

$$
H^{i}\left(F, \mathbb{G}_{a}\right) \simeq H_{H}^{i}\left(D, \mathbb{G}_{a}\right)=0 \text { pour } i>0,
$$

où $H_{H}$ désigne la cohomologie de Hochschild des schémas en groupes affines. En particulier, on a $\mathbb{R} S p e c \mathbb{L} \mathcal{O}(F) \simeq *$, et donc d'après le corollaire $2.2 .10 \mathrm{~F}$ n'est certainement pas un champ affine.

Cet exemple est assez représentatif de ce qui peut se passer, et en général il est bon de retenir que les faisceaux d'homotopie d'un champ affine auront tendence à être des schémas en groupes affines et unipotents (voir Thm. 2.4.5).

- Il existe des faisceaux d'ensembles qui sont des champs affines sans être représentables par des schémas affines. Par exemple, le plan épointé $\mathbb{A}^{2}-\{0\}$ est un champ affine. En effet, on peut écrire $\mathbb{A}^{2}-\{0\}$ comme le champ quotient $\left[S l_{2} / \mathbb{G}_{a}\right]$, ou en d'autres termes il existe une suite exacte de fibrations

$$
S l_{2} \longrightarrow \mathbb{A}^{2}-\{0\} \longrightarrow K\left(\mathbb{G}_{a}, 1\right) .
$$

On déduit aisément de ce diagramme et du lemme des cinqs que le morphisme induit $\mathbb{A}^{2}-\{0\} \longrightarrow \mathbb{L O}\left(\mathbb{A}^{2}-\{0\}\right)$ est un isomorphisme de champs. Le corollaire 2.2.10 montre que $\mathbb{A}^{2}-\{0\}$ est un champ affine.

Corollaire 2.2.11. Un préfaisceau simplicial sous-affine $F$, qui est une $\mathbb{V}$-limite homotopique de champs affines, est un champ affine.

Preuve: On invoque le théorème 2.2.9 et le fait qu'une $\mathbb{V}$-limite homotopique de préfaisceaux simpliciaux $\mathcal{O}$-locaux est encore $\mathcal{O}$-local.

\subsection{Affination des types d'homotopie}

Dans le paragraphe précédent nous avons défini la notion de champs affines. Nous allons maintenant définir la notion d'affination. Tout comme nous proposons les champs affines comme modèles aux complexes of unipotent bundles, nous proposons la notion d'affination comme réponse au problème de la schématisation de [18]. Nous résèrverons l'expression de schématisation pour la notion mieux adaptée au cas non-simplement connexe discutée dans le chapitre suivant.

Définition 2.3.1. Soit $F \in H o(S P r(k))$ un champ. Une affination de $F$, est la donnée d'un champ affine $(F \otimes k)^{u n i}$, et d'un morphism dans $\operatorname{Ho}(\operatorname{SPr}(k)), u$ : $F \longrightarrow(F \otimes k)^{u n i}$ qui soit universel pour les morphismes vers des champs affines.

Remarque: L'exposant uni dans la notation $(F \otimes k)^{u n i}$ fait référence au mot unipotent.

Commençons par remarquer que lorsqu'une affination existe elle est unique. De plus, comme les $K\left(\mathbb{G}_{a}, n\right)$ sont des champs affines, le morphisme $u$ dans la définition précédente est toujours une $\mathcal{O}$-équivalence. Ainsi, le théorème 2.2.9 montre qu'une affination est aussi une $\mathcal{O}$-localisation (i.e. une $\mathcal{O}$-equivalence vers un 
objet $\mathcal{O}$-local), et le morphisme $u$ dans la définition précédente est donc aussi universel pour les morphismes vers des objects $\mathcal{O}$-locaux.

Proposition 2.3.2. Soit $F \in H o(S P r(k))$ un champ. Pour qu'une affination de $F$ existe il faut et il suffit que $\mathbb{L O}(F)$ soit isomorphe dans $\mathrm{Ho}\left(k-A l g^{\Delta}\right)$ à un objet de $\mathbb{U}$.

De plus, pour un tel préfaisceau simplicial on a

$$
(F \otimes k)^{\text {uni }} \simeq \mathbb{R} \operatorname{Spec}(\mathbb{L} \mathcal{O}(F)) .
$$

Preuve: Il suffit de faire appel au corollaire 2.2.10.

Corollaire 2.3.3. Tout ensemble simplicial $X$ appartenant à $\mathbb{U}$ (et vu comme préfaisceau simplicial constant sur $\left.(A f f / k)_{f p q c}\right)$ possède une affination $(X \otimes k)^{\text {uni }}$. De plus, pour un tel ensemble simplicial $X$ on a

$$
(X \otimes k)^{u n i} \simeq \mathbb{R} \operatorname{Spec}\left(k^{X}\right),
$$

où $k^{X}$ est la $k$-algèbre co-simpliciale de cohomologie de $X$ à valeurs dans $k$.

Preuve: Le préfaisceau simplicial constant associé à un U-ensemble simplicial $X$ étant un objet cofibrant dans $\operatorname{SPr}(k)$, on a

$$
\mathbb{L} \mathcal{O}(X) \simeq \mathcal{O}(X) \simeq k^{X}
$$

Ceci montre que $\mathbb{L} \mathcal{O}(X)$ est bien isomorphe à un objet de $\mathbb{U}$, et termine la preuve.

Notons $H o(C A f f / k)$ la sous-catégorie pleine de $\operatorname{Ho}(\operatorname{PPr}(k))$ formée des champs affines sur $k$. En utilisant le corollaire 2.2.3, on voit que le foncteur dérivé des sections globales

$$
\mathbb{R} \Gamma: H o(S \operatorname{Pr}(k)) \longrightarrow H o(\mathbb{V}-S E n s)
$$

envoie $H o(C A f f / k)$ dans la sous-catégorie de $H o(\mathbb{V}-S E n s)$ formée des objets isomorphes à des $\mathbb{U}$-ensembles simpliciaux. Il se factorise donc en un foncteur

$$
\mathbb{R} \Gamma: H o(C A f f / k) \longrightarrow H o(\mathbb{U}-S E n s) .
$$

Corollaire 2.3.4. Le foncteur dérivé du foncteur des sections globales

$$
\mathbb{R} \Gamma: H o(C A f f / k) \longrightarrow H o(\mathbb{U}-S E n s)
$$

possède un adjoint à gauche

$$
(-\otimes k)^{u n i}: H o(\mathbb{U}-S E n s) \longrightarrow H o(C A f f / k) .
$$

Preuve: C'est une autre façon d'énoncer le corollaire 2.3.3.

Soit $u: k \longrightarrow k^{\prime}$ un morphisme d'anneaux dans $\mathbb{U}$. On peut alors définir un foncteur de restriction $u^{*}: S \operatorname{Pr}(k) \longrightarrow S \operatorname{Pr}\left(k^{\prime}\right)$. Il est défini par la formule suivante

$$
u^{*}(F)(\operatorname{Spec} A):=F(\operatorname{Spec} A)
$$


où $F \in S \operatorname{Pr}(k)$, et $A \in A f f / k^{\prime}$ qui est aussi considérée comme objet de $A f f / k$ via le morphisme $k \stackrel{u}{\longrightarrow} k^{\prime} \longrightarrow A$. Ce foncteur est clairement un foncteur de Quillen à droite qui de plus préserve les équivalences. Il induit donc un foncteur de changement de bases

$$
u^{*}: H o(S P r(k)) \longrightarrow H o\left(S P r\left(k^{\prime}\right)\right) .
$$

Nous utiliserons aussi la notation

$$
F \otimes_{k} k^{\prime}:=u^{*}(F) .
$$

Corollaire 2.3.5. Soit $u: k \longrightarrow k^{\prime}$ un morphisme de $\mathbb{U}$-anneaux, et $X$ un $\mathbb{U}$ ensemble simplicial fini. Alors, on a

$$
(X \otimes k)^{u n i} \otimes_{k} k^{\prime} \simeq\left(X \otimes k^{\prime}\right)^{u n i}
$$

Preuve: D'après la proposition 2.3.2 on a $(X \otimes k)^{\text {uni }} \simeq \mathbb{R} \operatorname{Spec}\left(k^{X}\right)$. Comme $k^{X}$ est une $k$-algèbre plate sur $k$, on a de plus

$$
k^{X} \coprod_{k}^{\mathbb{L}} k^{\prime} \simeq k^{X} \otimes_{k}^{\mathbb{L}} k^{\prime} \simeq\left(k^{\prime}\right)^{X},
$$

le second isomorphisme utilisant que $X$ est fini. Ainsi, on a pour tout $A \in A f f / k^{\prime}$

$$
\begin{aligned}
(X \otimes k)^{\text {uni }} \otimes_{k} k^{\prime} & \simeq \mathbb{R} \underline{\operatorname{Hom}}_{k^{\prime}-A_{l g} \Delta}\left(k^{X}, A\right) \simeq \mathbb{R}_{\underline{H o m}_{k^{\prime}-A l g^{\Delta}}\left(k^{X} \otimes_{k}^{\mathbb{L}} k^{\prime}, A\right)} \\
& \simeq \mathbb{R} \operatorname{Spec}\left(\left(k^{\prime}\right)^{X}\right)(A) \simeq\left(X \otimes k^{\prime}\right)^{\text {uni }}(A) .
\end{aligned}
$$

Le corollaire précédent montre que l'affination d'un $\mathbb{U}$-ensemble simplcial fini $X$ est stable par changement de bases, et donc que le champ $(X \otimes \mathbb{Z})^{\text {uni }}$ permet de déterminer les champs $(X \otimes k)^{u n i}$ pour tout anneau $k$, par la formule suivante

$$
(X \otimes k)^{u n i} \simeq(X \otimes \mathbb{Z})^{u n i} \otimes_{\mathbb{Z}} k .
$$

En général, il est impossible de décrire explicitement le champ $(X \otimes \mathbb{Z})^{\text {uni }}$, ni même ses faisceaux d'homotopie. Par contre, lorsque $X$ est simplement connexe et de type fini (i.e. tous ses groupes d'homotopie sont de type finis), on peut utiliser le théorème d'Eilenberg-Moore afin de calculer les faisceaux $\pi_{i}(X \otimes \mathbb{Z})$. Le procédé standard permet alors de se ramener au cas élémentaire où $X=K(\mathbb{Z}, n)$ (voir la preuve du théorème 2.5.1). Nous ne démontrerons rien à ce sujet dans cet article, et nous nous contenterons de donner une conjecture.

Pour toute $\mathbb{Z}$-algèbre $A$ dans $\mathbb{U}$, notons $\Lambda(A)$ le groupe additif des vecteurs de Witt universels à coefficients dans $A$ (voir $[11, V, \S 4,5.1 .1]$ ). En d'autres termes $\Lambda(A)$ est le groupe multiplicatif des séries formelles à coefficients dans $A$ et de la forme $1+a_{1} X+a_{2} X^{2}+\cdots+a_{n} X^{n}+\ldots$ Le foncteur Spec $A \mapsto \Lambda(A)$ est clairement représentable par un schéma en groupes affine sur $S p e c \mathbb{Z}$, qui sera encore noté $\Lambda$.

On définit un sous-foncteur $\mathbb{H}$ de $\Lambda$ de la façon suivante. Pour tout $A \in$ Aff $/ \mathbb{Z}$, une série formelle $P(X) \in 1+X A[[X]]=\Lambda(A)$ appartient à $\mathbb{H}(A)$ si et seulement si elle satisfait l'équation fonctionnelle $P(X) \cdot P(Y)=P(X+Y+X Y)$. Le foncteur $\mathbb{H}$ est un sous-schéma en groupes fermé dans $\Lambda$ et est donc aussi 
représentable par un schéma an groupes affine sur $S p e c \mathbb{Z}$. Le schéma en groupes $\mathbb{H}$ a été introduit dans [44, App. $C$ ] pour les besoins du calcul du complété proalgébrique du groupe $\mathbb{Z}$, où il est implicitement identifié au complété unipotent de $\mathbb{Z}$ sur $S$ pec $\mathbb{Z}$. On peut justifier ce point de vue en observant que $\mathbb{H}$ est aussi le schéma en groupes des automorphismes monoïdaux du foncteur d'oubli de la catégorie des $\mathbb{Z}$-modules libres de rang fini munis d'un automorphisme unipotent.

Pour tout entier $n \in \mathbb{Z}$, on peut associér la série formelle $(1+X)^{n} \in \mathbb{H}(\mathbb{Z})$. Ceci définit un morphisme de préfaisceaux en groupes $\mathbb{Z} \longrightarrow \mathbb{H}$. On en déduit donc un morphisme de préfaisceaux simpliciaux $K(\mathbb{Z}, n) \longrightarrow K(\mathbb{H}, n)$.

Conjecture 2.3.6. Le champ $K(\mathbb{H}, n)$ est affine et le morphisme précédemment défini $K(\mathbb{Z}, n) \longrightarrow K(\mathbb{H}, n)$ est une affination.

Une réponse positive à la question précédente impliquerait que pour tout U-ensemble simplicial $X$, qui est simplement connexe et de type fini, on ait

$$
\pi_{i}\left((X \otimes \mathbb{Z})^{u n i}\right) \simeq \pi_{i}(X) \otimes_{\mathbb{Z}} \mathbb{H} .
$$

En d'autres termes, les faisceaux d'homotopie de $X$ sont les complétés unipotents sur $S p e c \mathbb{Z}$ des groupes d'homotopie de $X$.

\subsection{Champs affines sur un corps}

Dans ce paragraphe nous allons étudier les champs affines et connexes lorsque $k$ est un corps. Il est remarquable que l'on possède alors une caractérisation des champs affines à l'aide de leurs faisceaux d'homotopie. Ce critère permet une grande libérté de manipulation, et il permettra en particulier de démontrer un critère analogue pour les types d'homotopie schématiques qui seront introduits dans le chapitre suivant. Ce critère jouera par ailleurs un rôle essentiel dans la preuve de l'existence du foncteur de schématisation. Enfin, les résultats de ce paragraphe confortent aussi le point de vue que les champs affines sont un modèle pour les complexes of unipotent bundles de [18].

Pour tout ce paragraphe $k$ sera un corps de caractéristique quelconque.

Nous commencerons par le théorème suivant. Pour la notion de schémas en groupes unipotents on renvoie le lecteur à $[11, I V, \S 2]$, ou encore au bref résumé se trouvant au paragraphe 1.5 .

Théorème 2.4.1. Soit $* \longrightarrow F$ un préfaisceau simplicial pointé et connexe tel que pour tout entier $i>0$ le faisceau en groupes $\pi_{i}(F, *)$ soit représentable par un schéma en groupes affine et unipotent. Alors $F$ est un champ affine.

Preuve: Pour réduire le problème nous allons systématiquement appliquer la proposition 2.2.7. Le preuve du théorème consiste donc à montrer que le champ $F$ s'écrit comme une $\mathbb{U}$-limite homotopique de champs de la forme $K\left(\mathbb{G}_{a}, n\right)$. Pour cela, commençons par montrer que l'on est sous les hypothèses de la proposition 
1.2.2 (et que l'on peut prendre $N=0$ ). Plus précisémment nous allons montrer que pour tout entier $i$, et tout schéma affine $X$, on a $H^{p}\left(X, \pi_{i}(F, *)\right)=0$ pour $p>1$ (il s'agit ici de cohomologie pour la topologie $f p q c$ ).

Pour tout entier $n$, notons $\pi_{i}(F, *)_{n}$ le sous-groupe fermé de $\pi_{i}(F, *)$ image du $n$-ème morphisme de décalage (voir $[11, I V, \S 3$ No. 4]) (si $k$ est de caractéristique nulle on prendra $\pi_{i}(F, *)_{n}=0$ pour tout $\left.n>0\right)$. Comme $\pi_{i}(F, *)$ est un schéma en groupes unipotent l'intersection des $\pi_{i}(F, *)_{n}$ est nulle, et il existe un isomorphisme de schémas en groupes affines

$$
\pi_{i}(F, *) \simeq \operatorname{Lim}_{n} M_{n},
$$

où $M_{n}:=\pi_{i}(F, *) / \pi_{i}(F, *)_{n}$. On dispose alors d'une suite exacte de Milnor

$$
0 \longrightarrow \operatorname{Lim}_{n}^{1} H^{p-1}\left(X, M_{n}\right) \longrightarrow H^{p}\left(X, \pi_{i}(F)\right) \longrightarrow \operatorname{Lim}_{n} H^{p}\left(X, M_{n}\right) \longrightarrow 0 .
$$

Cette suite exacte montre en particulier qu'il suffit de montrer que pour tout $p>1$ et tout entier $n$ on a $H^{p}\left(X, M_{n}\right)=0$. En effet, dans ce cas les morphismes de transitions du système projectif $\left\{H^{p-1}\left(X, M_{n}\right)\right\}_{n}$ seront tous surjectifs pour $p>1$, et ainsi le terme $\operatorname{Lim}_{n}^{1} H^{p-1}\left(X, M_{n}\right)$ sera nul pour $p>1$.

On se ramène ainsi au cas où $\pi_{i}(F, *)_{n}=0$ pour un certain $n$. De plus, à l'aide de la suite exacte longue en cohomologie on peut même supposer que $\pi_{i}(F, *)_{1}=0$, ou en d'autres termes que $\pi_{i}(F, *)$ est annulé par le décalage. Dans ce cas, on sait qu'il existe une suite exacte courte de schémas en groupes (voir [11, IV §3 Thm. $6,6]$ et $[11, I V \S 3$ Cor. 6.8])

$$
0 \longrightarrow \pi_{i}(F, *) \longrightarrow \mathbb{G}_{a}^{I} \longrightarrow \mathbb{G}_{a}^{J} \longrightarrow 0 .
$$

Or, comme chaque $\mathbb{G}_{a}$ est le faisceau en groupes sous-jacent à un $\mathcal{O}$-module quasicohérent, on a pour $p>0$

$$
H^{p}\left(X, \mathbb{G}_{a}^{I}\right) \simeq H^{p}\left(X, \mathbb{G}_{a}\right)^{I} \simeq 0,
$$

et on voit à l'aide de la suite exacte longue en cohomologie que ceci implique que $H^{p}\left(X, \pi_{i}(F, *)\right) \simeq 0$ pour $p>1$.

On vient de voir que les hypothèses de 1.2.2 étaient satisfaites, et donc que $F$ est équivalent à la limite homotopique de sa tour de Postnikov. Ainsi, $F$ est équivalent à la limite projective homotopique d'un diagramme $\left\{F_{n}\right\}_{n}$, avec $F_{0}=*$, $F_{1}=K\left(\pi_{1}(F, *), 1\right)$, et où il existe des diagrammes homotopiquement cartésiens dans $\operatorname{SPr}(k)$

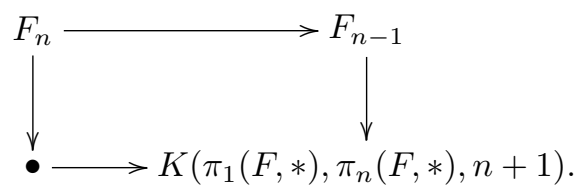

Comme une $\mathbb{U}$-limite homotopique de champs affines est un champ affine, la discussion précédente montre que l'on peut supposer que $F=K(G, M, n)$, pour $G$ un schéma en groupes affine unipotent opérant sur un schéma en groupes 
affine unipotent abélien $M$. Notons alors $M_{j}$ le sous-groupe de $M$ image du $j$-ème morphisme de décalage. Comme les sous-groupes $M_{j}$ sont caractéristiques, on en déduit une filtration $G$-équivariante

$$
\ldots M_{j} \subset M_{j-1} \subset \ldots M_{1} \subset M_{0}=M .
$$

De plus, comme $M$ est un schéma en groupes unipotent, le morphisme naturel $M \longrightarrow \operatorname{Lim}_{j} M / M_{j}$ est un isomorphisme $G$-équivariant.

Lemme 2.4.2. Le morphisme naturel

$$
K(G, M, n) \longrightarrow \operatorname{Holim}_{j} K\left(G, M / M_{j}, n\right)
$$

est une équivalence.

Preuve: Le diagramme commutatif suivant

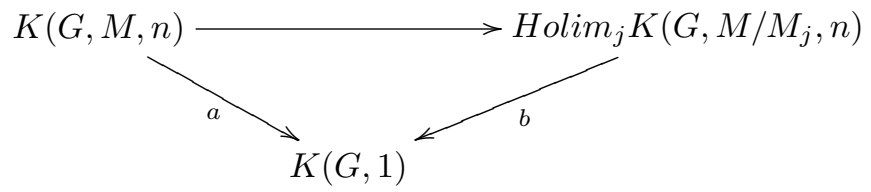

montre qu'il suffit de démontrer que le morphisme induit sur les fibres homotopiques de $a$ et $b$ est une équivalence. En d'autres termes on peut supposer que $G$ est trivial. Soit $E$ (resp. $\left.E_{j}\right)$ un modèle fibrant de $K(M, n)\left(\operatorname{resp} . \operatorname{de} K\left(M / M_{j}, n\right)\right)$. Comme les groupes $M$ et $M / M_{j}$ sont des schémas en groupes unipotents on a déjà vu que $\pi_{i}^{p r}(E)=\pi_{i}^{p r}\left(E_{j}\right)=0$ pour $i \neq n, n-1$. On utilise alors la suite exacte courte de Milnor

$$
0 \longrightarrow \operatorname{Lim}_{j}^{1} \pi_{i+1}^{p r}\left(E_{j}\right) \longrightarrow \pi_{i}^{p r}\left(\operatorname{Holim}_{j} E_{j}\right) \longrightarrow \operatorname{Lim}_{j} \pi_{i}^{p r}\left(E_{j}\right) \longrightarrow 0 .
$$

Cette suite exacte montre en particulier que $\pi_{i}\left(\right.$ Holim $\left._{j} E_{j}\right)=0$ pour $i<n-2$. De plus, pour $i=n-1$, on trouve $\pi_{n-2}^{p r}\left(\operatorname{Holim}_{j} E_{j}\right) \simeq \operatorname{Lim}_{j}^{1} \pi_{n-1}^{p r}\left(E_{j}\right)$. En d'autres termes, pour tout $X \in C$ on a

$$
\pi_{n-2}\left(\operatorname{Holim}_{j} E_{j}(X)\right) \simeq \operatorname{Lim}_{j}^{1} \pi_{n-1}\left(E_{j}(X)\right) \simeq \operatorname{Lim}_{j}^{1} H^{1}\left(X, M / M_{j}\right) .
$$

Or, comme $M / M_{j} \longrightarrow M / M_{j-1}$ est surjectif, et que $H^{2}\left(X, M / M_{j}\right)=0$ pour tout $j$, les morphismes de transitions $H^{1}\left(X, M / M_{j}\right) \longrightarrow H^{1}\left(X, M / M_{j-1}\right)$ sont surjectifs. Ainsi, on a $\operatorname{Lim}_{j}^{1} H^{1}\left(X, M / M_{j}\right)=0$. On en déduit que $\pi_{n-2}\left(\operatorname{Holim}_{j} E_{j}\right)$ est trivial, et donc que $\mathrm{Holim}_{j} E_{j}$ est $(n-2)$-connexe. On peut donc invoquer le théorème 1.4.3 pour se ramener au cas où $n=1$. On conclut alors à l'aide du lemme 2.4.4 (on vérifiera qu'il n'y a pas d'argument circulaire).

Le lemme précédent permet de se ramener au cas où $M_{j_{0}}$ est nul pour un certain $j_{0}$. On dispose dans ce cas de diagrammes homotopiquement cartésiens 
dans $\operatorname{SPr}(k)$

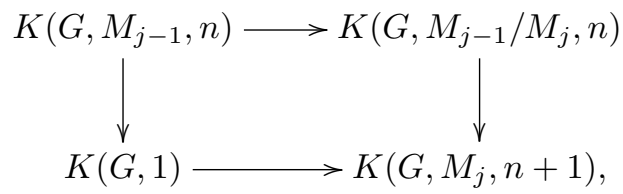

qui montrent que l'on peut réduire le problème par récurrence au cas où $M$ est annulé par le morphisme de décalage.

Soit $M^{*}:=\underline{\operatorname{Hom}}_{g p}\left(M, \mathbb{G}_{a}\right)$ le $\mathcal{O}$-module quasi-cohérent des morphismes de schémas en groupes de $M$ vers $\mathbb{G}_{a}$. Le groupe $G$ opère linéairement sur $M^{*}$, ainsi que sur son $\mathcal{O}$-module dual $V(M):=\underline{\operatorname{Hom}}_{\mathcal{O}}\left(M^{*}, \mathcal{O}\right)$. De plus, comme $M$ est annulé par le décalage, il peut-être réalisé comme un sous-groupe fermé d'un produit de $\mathbb{G}_{a}$, et ceci implique que le morphisme de bidualité

$$
M \longrightarrow V(M)
$$

est un monomorphisme $G$-équivariant de schémas en groupes. Enfin, comme $V(M)$ est lui-même un schéma en groupes unipotent annulé par le décalage (car il est isomorphe à un produit de $\mathbb{G}_{a}$ ), on peut poursuivre le procédé et construire une résolution co-simpliciale $G$-équivariante

$$
M \longrightarrow V_{*}(M),
$$

où chaque $V_{n}(M)$ est le schéma en groupes dual d'une représentation linéaire de $G$.

Lemme 2.4.3. Le morphisme naturel

$$
K(G, M, n) \longrightarrow \text { Holim }_{p \in \Delta} K\left(G, V_{p}(M), n\right)
$$

est une équivalence dans $S \operatorname{Pr}(k)$.

Preuve: En considérant le diagramme commutatif suivant

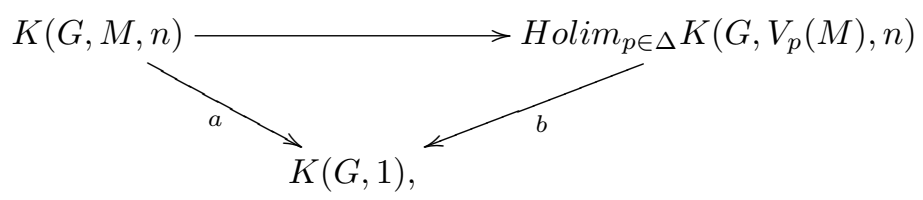

on voit qu'il suffit de montrer que le morphisme induit sur les fibres homotopiques de $a$ et $b$ est une équivalence. En d'autres termes on peut supposer que $G$ est trivial.

Il est facile de voir que le morphisme $K(M, n) \longrightarrow \operatorname{Holim}_{p \in \Delta}^{t r v i} K\left(V_{p}(M), n\right)$ est une équivalence. De plus, comme chaque $V_{p}(M)$ est un produit de faisceaux quasicohérents, il est acyclique, et donc tous les préfaisceaux simpliciaux $K\left(V_{p}(M), n\right)$ sont des champs. Le lemme se déduit donc du lemme 1.2.1.

Le lemme précédent permet de se ramener au cas où $M$ est le $\mathcal{O}$-module dual d'une représentation linéaire de $G$. Comme une telle représentation est limite 
inductive de ses sous-représentations de dimension finie, on peut écrire $M$ sous la forme d'une limite projective de représentations linéaires de dimension finie

$$
M \simeq \operatorname{Lim}_{i} M_{i}
$$

Par un argument assez similaire à celui utilisé lors de la preuve du lemme précédent on montre qu'il existe une équivalence

$$
K(G, M, n) \simeq \operatorname{Holim}_{i} K\left(G, M_{i}, n\right)
$$

(on utilisera par exemple que le foncteur $\operatorname{Lim}_{i}$ est exact dans la category des $k$ espaces vectoriels linéairement compacts). Ceci permet donc de se ramener au cas où $M$ est une représentation linéaire de dimension finie de $G$. Comme le schéma en groupes $G$ est unipotent, il existe une suite de sous-représentations linéaires $0=M_{r} \subset M_{r-1} \subset \cdots \subset M_{1} \subset M_{0}=M$, tel que $G$ opère trivialement sur chaque quotient $M_{i} / M_{i+1}$. Chaque champ $K\left(M_{i} / M_{i+1}, n\right)$ est alors la fibre homotopique du morphisme naturel $K\left(G, M / M_{i}, n\right) \longrightarrow K\left(G, M / M_{i+1}, n\right)$. Il existe donc des diagrammes homotopiquement cartésiens dans $\operatorname{SPr}(k)$

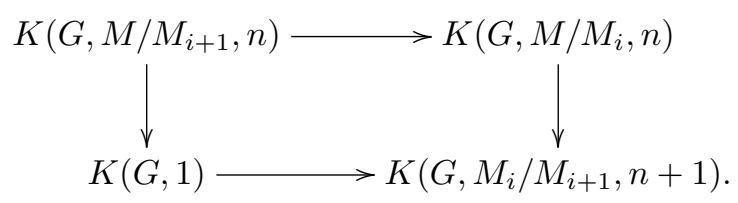

On se ramène ainsi par une récurrence au cas où $F=K(G, 1)$ et au cas où $F=K(G, M, n)$ et $G$ opère trivialement sur $M$. Dans ce cas, on a $F=K(G, 1) \times$ $K(M, n)$ et il nous reste à montrer que chacun des facteurs est affine.

Pour le facteur $K(M, n)$, c'est immédiat car $M$ est isomorphe à un produit fini de $\mathbb{G}_{a}$. Enfin, pour le facteur $K(G, 1)$ on écrit $G$ comme la limite projective de ses quotients de type fini sur $k$, ce qui permet à l'aide du lemme suivant de supposer que $G$ est de type fini.

Lemme 2.4.4. Soit $G$ un schéma en groupe affine, et $\left\{G_{i}\right\}_{i \in I}$ le système projectif de ses quotients qui sont de type fini sur $k$. Alors, le morphisme naturel

$$
K(G, 1) \longrightarrow \text { Holim }_{i \in I} K\left(G_{i}, 1\right)
$$

est une équivalence.

Preuve: Soit $E$ (resp. $\left.E_{i}\right)$ un modèle fibrant pour $K(G, 1)\left(\operatorname{resp} . K\left(G_{i}, 1\right)\right)$. On peut prendre par exemple pour $E$ (resp. pour $E_{i}$ ) le champ des $G$-torseurs (resp. des $G_{i}$-torseurs). Il s'agit alors de montrer que pour tout schéma affine $X$, le groupoïde des $G$-torseurs sur $X$ est équivalent à la limite homotopique des groupoïdes des $G_{i}$-torseurs sur $X$. Ce qui est vrai.

Lorsque $G$ est de type fini on utilise qu'il possède une suite de composition centrale dont les quotients succesifs sont des sous-groupes fermés de $\mathbb{G}_{a}$ (voir $[11, I V, \S 2$, Prop.2.5]). Il existe une suite de composition centrale de sous-groupes 
fermés $1=G_{r} \subset G_{r-1} \subset \cdots \subset G_{0}=G$, et des diagrammes homotopiquement cartésiens

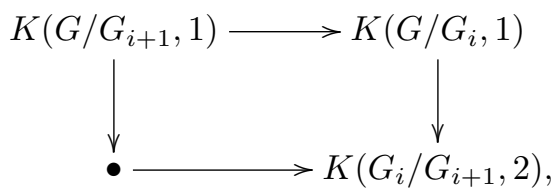

tel que chaque $G_{i} / G_{i+1}$ soit un sous-groupe fermé de $\mathbb{G}_{a}$. Par récurrence il nous suffit de montrer que pour tout sous-groupe fermé $G \subset \mathbb{G}_{a}$, le champ $K(G, 2)$ est affine. Mais dans ce cas, ou bien $G=\mathbb{G}_{a}$, ou bien il existe une suite exacte courte de schémas en groupes

$$
0 \longrightarrow G \longrightarrow \mathbb{G}_{a} \longrightarrow \mathbb{G}_{a} \longrightarrow 0 .
$$

Dans les deux cas, ceci implique que $K(G, 2)$ est affine.

Nous allons maintenant donner une réciproque au théorème 2.4.1. Pour cela nous allons étudier les faisceaux d'homotopie des champs de la forme $\mathbb{R} S p e c A$, où $A$ est une $k$-algèbre co-simpliciale augementée $A \longrightarrow k$, et cohomologiquement connexe (i.e. $H^{0}(A) \simeq k$ ). Le théorème suivant peut être interprété comme une version géométrique de l'existence des modèles minimaux (voir [4, 34]).

Théorème 2.4.5. Soit $A$ une $k$-algèbre co-simpliciale de $\mathbb{U}$, augmentée $A \longrightarrow k$, et cohomologiquement connexe. Notons Speck $=* \longrightarrow \mathbb{R}$ Spec $A=F$ le champ pointé associé. Alors, pour tout $i>0$, le faisceau $\pi_{i}(F, *)$ est représentable par un schéma en groupes affine et unipotent. De plus, le champ F est connexe (i.e. $\left.\pi_{0}(F)=*\right)$.

Preuve: Il s'agit en réalité de construire une décomposition de Postnikov de la $k$-algèbre co-simpliciale $A$. Dans le cas où $A$ est 1 -connexe (i.e. on a de plus $\left.H^{1}(A)=0\right)$, l'existence de cette décomposition est démontrée dans [34, 3.2].

La preuve consiste à montrer qu'il existe une tour de champs pointés

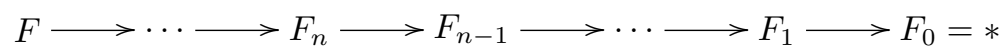

vérifiant les trois propriétés suivantes.

- Le champ $F_{n}$ est connexe et $n$-tronqué (i.e. $\pi_{i}\left(F_{n}, *\right)=0$ pour tout $\left.i>n\right)$. De plus, le morphisme naturel $F_{n} \longrightarrow F_{n-1}$ induit une isomorphisme de champs $\tau_{\leq n-1} F_{n} \simeq F_{n-1}$.

- Pour tout $n$ et tout $i$, les faisceaux $\pi_{i}\left(F_{n}, *\right)$ sont représentables par des schémas en groupes affines et unipotents.

- Le morphisme $f_{n}^{*}: H^{i}\left(F_{n}, \mathbb{G}_{a}\right) \longrightarrow H^{i}\left(F, \mathbb{G}_{a}\right)$ est bijectif pour $i \leq n$, et injectif pour $i=n+1$.

En effet, supposons l'existence d'une telle tour et démontrons le théorème.

Lemme 2.4.6. Le champ Holim $F_{n}$ est affine. 
Preuve: Chaque $F_{n}$ est un champ affine d'après le théorème 2.4.1. Ainsi, le lemme se déduit de la proposition 2.2.7.

Lemme 2.4.7. Le morphisme $F \longrightarrow \operatorname{Holim}_{n} F_{n}$ est une $\mathcal{O}$-équivalence.

Preuve: Comme les champs $F_{n}$, ainsi que $\operatorname{Holim}_{n} F_{n}$, sont des champs affines, on a d'après le corollaire 2.2.3

$$
\mathbb{L} \mathcal{O}\left(\operatorname{Holim}_{n} F_{n}\right) \simeq \text { Hocolim }_{n} \mathbb{L} \mathcal{O}\left(F_{n}\right) .
$$

Comme d'après le corollaire 2.2.6 on a

$$
H^{i}\left(\operatorname{Hocolim}_{n} \mathbb{L} \mathcal{O}\left(F_{n}\right)\right) \simeq \operatorname{Colim}_{n} H^{i}\left(F_{n}, \mathbb{G}_{a}\right),
$$

le lemme se déduit alors immédiatement de la troisième propriété de la tour $\left\{F_{n}\right\}_{n}$.

Les deux lemmes précédents impliquent que le morphisme $F \longrightarrow \operatorname{Holim}_{n} F_{n}$ est un isomorphism. Remarquons de plus que chaque faisceau $\pi_{i}\left(F_{n}, *\right)$ est un schéma en groupes unipotent, et donc de dimension cohomologique inférieure à 1 . Le corollaire 1.2.3 permet alors de conclure.

Il nous reste maintenant à démontrer l'existence de la tour de champs

$$
F \longrightarrow \cdots \longrightarrow F_{n} \longrightarrow F_{n-1} \longrightarrow \cdots \longrightarrow F_{1} \longrightarrow F_{0}=*
$$

vérifiant les trois conditions précédentes. Nous allons procéder par une double récurrence. Supponsons pour cela que nous ayons défini les $F_{i}$ pour $i<n+1$, et définissons $F_{n+1}$ de la façon suivante (cela va prendre un certain temps).

Commençons par quelques mots sur la notion de faisceaux d'homologie de champs. Pour tout champ $F$, le groupe $H^{i}\left(F, \mathbb{G}_{a}\right)$ est muni de l'endomorphisme induit par l'endomorphisme de Frobenius $\mathbf{F}: \mathbb{G}_{a} \longrightarrow \mathbb{G}_{a}$. Plus précisement, pour tout $\operatorname{Spec} A \in A f f / k$, on a $\mathbf{F}(a):=a^{p}$ pour $a \in A=\mathbb{G}_{a}(\operatorname{Spec} A)$, et où $p$ est l'exposant caractéristique de $k$ (ainsi, $\mathbf{F}=I d$ si $k$ est de caractéristique nulle). Nous noterons $B$ l'anneau des endomorphismes de groupes de $\mathbb{G}_{a}$. Ainsi, si car $k=p>0$, $B \simeq k[\mathbf{F}]$ est l'anneau des polynômes non-commutatifs avec un seul générateur $\mathbf{F}$, et une seule relation F. $a:=a^{p} . \mathbf{F}$. En contre partie, si car $k=0$, alors $B \simeq k$. Nous verrons donc les groupes $H^{i}\left(F, \mathbb{G}_{a}\right)$ comme des $B$-modules.

Rappelons alors que tout $B$-module $M$ définit un faisceau en groupes abélien

$$
\begin{array}{ccc}
H(M): \quad(A f f / k)_{f p q c} & \longrightarrow & A b \\
\operatorname{Spec} A & \mapsto & \operatorname{Hom}_{B}(M, A),
\end{array}
$$

où toute $k$-algèbre $A$ est vue comme un $B$-module par l'action $\mathbf{F}(a)=a^{p}$, pour $a \in A$. D'après [11, $I V, \S 3$, Cor. 6.7], le foncteur $H$ définit une équivalence entre la catégorie des $B$-modules appartenant à $\mathbb{U}$, et celle des schémas en groupes unipotents abéliens et annulés par le décalage (i.e. qui sont sous-groupes fermés d'un produit $\mathbb{G}_{a}^{J}$, avec $\left.J \in \mathbb{U}\right)$. 
Nous noterons $H_{i}(F):=H\left(H^{i}\left(F, \mathbb{G}_{a}\right)\right)$, que nous appellerons les faisceaux d'homologie de $F$. Ce sont des schémas en groupes unipotents abéliens dès que $H^{i}\left(F, \mathbb{G}_{a}\right)$ est isomorphe à un object de $\mathbb{U}$ (e.g. si $F$ est affine). Un exemple fondamental est $K(H, n)$, avec $H$ un schéma en groupes unipotent, abélien et annulé par le décalage. Dans ce cas il est facile de vérifier que l'on a $H_{n}(K(H, n)) \simeq H$. Ainsi, pour un tel schéma en groupes $H$, on dispose par fonctorialité d'un morphisme

$$
H^{n}(F, H) \simeq \pi_{0} \underline{\mathbb{R}} \underline{\operatorname{Hom}}(F, K(H, n)) \longrightarrow \operatorname{Hom}\left(H_{n}(F), H\right) .
$$

Nous aurons alors besoin du lemme suivant, reliant homologie et cohomologie.

Lemme 2.4.8. Soit $F$ un champ affine, et $H$ un schéma en groupes unipotent, abélien et annulé par le décalage. Alors le morphisme naturel

$$
H^{n}(F, H) \longrightarrow \operatorname{Hom}\left(H_{n}(F), H\right)
$$

est surjectif.

Preuve: Soit $M$ un $B$-module tel que $H \simeq H(M)$, et

$$
0 \longrightarrow B^{J} \longrightarrow B^{I} \longrightarrow M \longrightarrow 0
$$

une résolution libre du $B$-module $M$. Elle correspond à une suite exacte de faisceaux en groupes

$$
0 \longrightarrow H(M) \longrightarrow \mathbb{G}_{a}^{I} \longrightarrow \mathbb{G}_{a}^{J} \longrightarrow 0 .
$$

On en déduit un diagramme commutatif

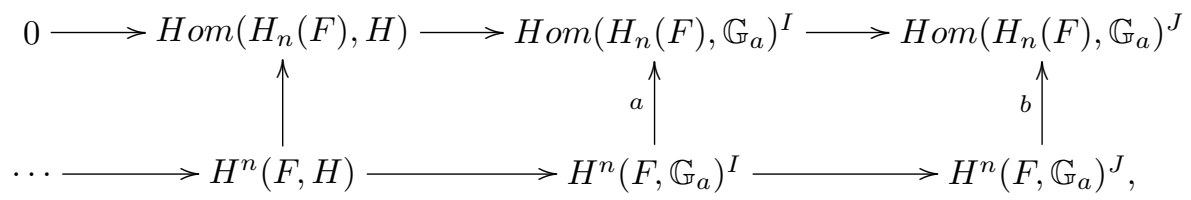

où $a$ et $b$ sont des isomorphismes $\left(\operatorname{car} \operatorname{Hom}\left(-, \mathbb{G}_{a}\right)\right.$ est le foncteur inverse de $\left.H\right)$. Ce diagramme montre que le morphisme en question est surjectif.

Nous allons commencer par construire une tour de champs affines, pointés et connexes

$$
F \longrightarrow \cdots \longrightarrow F_{n, q} \longrightarrow F_{n, q-1} \longrightarrow \cdots \longrightarrow F_{n, 1} \longrightarrow F_{n, 0}=F_{n},
$$

puis poser $F_{n+1}:=\operatorname{Holim}_{q} F_{n, q}$. Les champs $F_{n, q}$ seront définis par récurrence de sorte à ce que la fibre homotopique de $F_{n, q+1} \longrightarrow F_{n, q}$ soit de la forme $K\left(\pi_{n+1, q+1}, n+1\right)$, avec $\pi_{n+1, q+1}$ un schéma en groupes abélien et unipotent. On montrera de plus par récurrence que le morphisme induit $H^{n+1}\left(F_{n, q}, \mathbb{G}_{a}\right) \longrightarrow$ $H^{n+1}\left(F, \mathbb{G}_{a}\right)$ est injectif. Le lecteur pourra comparer la consrtuction des $F_{n, q}$ décrite ci-dessous avec la construction du modèle minimal dans [4, Prop. 7.7].

Supposons $F \longrightarrow F_{n, q} \longrightarrow F_{n}$ construit, et considérons la suite exacte courte

$$
0 \longrightarrow H^{n+1}\left(F_{n, q}, \mathbb{G}_{a}\right) \longrightarrow H^{n+1}\left(F, \mathbb{G}_{a}\right) \longrightarrow C \text {. }
$$


L'endomorphisme de Frobenius $\mathbf{F}: \mathbb{G}_{a} \longrightarrow \mathbb{G}_{a}$ induit un endomorphisme de la suite exacte précédente. Cette suite exacte peut donc être considérée comme une suite exacte de $B$-modules, ou encore à travers le foncteur $H$ comme une suite exacte courte de schémas en groupes abéliens et unipotents

$$
0 \longrightarrow H(C) \longrightarrow H_{n+1}(F) \longrightarrow H_{n+1}\left(F_{n, q}\right) \longrightarrow 0 .
$$

Cette suite exacte de faisceaux abéliens est classifiée par un morphisme dans la catégorie dérivée des faisceaux abéliens sur $(A f f / k)_{f p q c}$

$$
e: H_{n+1}\left(F_{n, q}\right)[n+1] \longrightarrow H(C)[n+2] .
$$

En oubliant la structure de groupes, ce morphisme induit à travers la correspondance de Dold-Kan un morphisme de champs

$$
e: K\left(H_{n+1}\left(F_{n, q}\right), n+1\right) \longrightarrow K(H(C), n+2) .
$$

De plus, le lemme 2.4.8 montre que l'on peut trouver un morphisme de champs $F_{n, q} \longrightarrow K\left(H_{n+1}\left(F_{n, q}\right), n+1\right)$ qui relève l'identité de $H_{n+1}\left(F_{n, q}\right)$. Composé avec le morphisme d'extension $e$, on en déduit un morphisme de champs

$$
F_{n, q} \longrightarrow K(H(C), n+2),
$$

dont la fibre homotopique sera notée $\widetilde{F_{n, q}} \longrightarrow F_{n, q}$. Le morphisme $F \longrightarrow F_{n, q} \longrightarrow$ $K(H(C), n+2)$ étant trivial dans $H o(S \operatorname{Pr}(k))$, on en déduit un morphisme $F \longrightarrow$ $\widetilde{F_{n, q}}$ qui relève $F \longrightarrow F_{n, q}$. Il est alors facile de vérifier que le morphisme induit

$$
H^{n+1}\left(\widetilde{F_{n, q}}, \mathbb{G}_{a}\right) \longrightarrow H^{n+1}\left(F, \mathbb{G}_{a}\right)
$$

est un isomorphisme.

Considérons maintenant la suite exacte de $B$-modules

$$
0 \longrightarrow K \longrightarrow H^{n+2}\left(\widetilde{F_{n, q}}, \mathbb{G}_{a}\right) \longrightarrow H^{n+2}\left(F, \mathbb{G}_{a}\right),
$$

et la suite de schémas en groupes abéliens unipotents associée

$$
H_{n+2}(F) \longrightarrow H_{n+2}\left(\widetilde{F_{n, q}}\right) \longrightarrow H(K) \longrightarrow 0 .
$$

A l'aide du lemme 2.4 .8 choississons un morphisme $\widetilde{F_{n, q}} \longrightarrow K(H(K), n+2)$ induisant la projection $H_{n+2}\left(\widetilde{F_{n, q}}\right) \longrightarrow H(K)$, et définissons $F_{n, q+1}$ comme étant sa fibre homotopique. Comme précédemment, le morphisme $F \longrightarrow \widetilde{F_{n, q}}$ se factorise par $F_{n, q+1}$. De plus, par construction il est facile de voir que le morphisme induit $H^{n+2}\left(F_{n, q+1}, \mathbb{G}_{a}\right) \longrightarrow H^{n+2}\left(F, \mathbb{G}_{a}\right)$ envoit $K \subset H^{n+2}\left(\widetilde{F_{n, q}}, \mathbb{G}_{a}\right)$ sur $0 \in H^{n+2}\left(F, \mathbb{G}_{a}\right)$. Pour finir, le lemme suivant montre que le morphisme induit $H^{n+1}\left(F_{n, q+1}, \mathbb{G}_{a}\right) \longrightarrow H^{n+1}\left(F, \mathbb{G}_{a}\right)$ est injectif, et ceci termine la construction du champ $F_{n, q+1}$. 
Lemme 2.4.9. Soit $p: F^{\prime} \longrightarrow F$ un morphisme entre deux champs affines pointés et connexes, dont la fibre homotopique est isomorphe à $K(\pi, n+1)$. Alors, le morphisme induit en cohomologie

$$
p^{*}: H^{n+1}\left(F, \mathbb{G}_{a}\right) \longrightarrow H^{n+1}\left(F^{\prime}, \mathbb{G}_{a}\right)
$$

est injectif.

Preuve: En utilisant les corollaires 2.2 .3 et 2.2.6, le morphisme $p$ est induit par un morphisme de $k$-algèbres co-simpliciales augmentées et cohomologiquement connexes $u: A \longrightarrow B$. Soit $C:=k \otimes_{A}^{\mathbb{L}} B$ la cofibre homotopique de $u$ le long de l'augmentation $A \longrightarrow k$. Alors, comme $K(\pi, n+1) \simeq \mathbb{R} \operatorname{Spec} C$, on a $H^{i}(C)=0$ pour $0<i<n+1$. Les premiers termes non-nuls de la suite spectrale d'EilenbergMoore (voir [34, 2.2]) donnent donc une suite exacte

$$
0 \longrightarrow H^{n+1}(A) \longrightarrow H^{n+1}(B) \longrightarrow \cdots
$$

D'après le corollaire 2.2.6 ceci implique le lemme.

En appliquant le corollaire 2.2.3, et le fait que les colimites filtrantes commutent avec les foncteurs de cohomologie dans, on trouve des isomorphismes

$$
H^{i}\left(F_{n+1}, \mathbb{G}_{a}\right) \simeq \operatorname{Colim}_{q} H^{i}\left(F_{n, q}, \mathbb{G}_{a}\right) \simeq \operatorname{Colim}_{q} H^{i}\left(\widetilde{F_{n, q}}, \mathbb{G}_{a}\right) .
$$

Comme chaque morphism $H^{n+1}\left(\widetilde{F_{n, q}}, \mathbb{G}_{a}\right) \longrightarrow H^{n+1}\left(F, \mathbb{G}_{a}\right)$ est un isomorphisme, on en déduit que le morphisme $H^{n+1}\left(F_{n+1}, \mathbb{G}_{a}\right) \longrightarrow H^{n+1}\left(F, \mathbb{G}_{a}\right)$ est un isomorphisme.

De plus, si $x \in \operatorname{Ker}\left(H^{n+2}\left(F_{n+1}, \mathbb{G}_{a}\right) \longrightarrow H^{n+2}\left(F, \mathbb{G}_{a}\right)\right)$, on peut représenter $x$ par un élément dans le noyau de $H^{n+2}\left(\widetilde{F_{n, q}}, \mathbb{G}_{a}\right) \longrightarrow H^{n+2}\left(F, \mathbb{G}_{a}\right)$. Par construction de $F_{n, q+1}$, ceci implique que l'image de $x$ dans $H^{n+2}\left(F_{n, q+1}, \mathbb{G}_{a}\right)$ est nulle. Ainsi, $x=0 \in H^{n+2}\left(F_{n+1}, \mathbb{G}_{a}\right)$. Le morphisme $H^{n+2}\left(F_{n+1}, \mathbb{G}_{a}\right) \longrightarrow H^{n+2}\left(F, \mathbb{G}_{a}\right)$ est donc injectif. Il nous reste ainsi à démontrer que $F_{n+1}$ est $(n+1)$-tronqué, que le morphisme $\tau_{\leq n} F_{n+1} \longrightarrow F_{n}$ est un isomorphisme, et que $\pi_{n+1}\left(F_{n+1}, *\right)$ est représentable par un schéma en groupes unipotent.

Commençons par montrer par récurrence sur $q$ que $F_{n, q}$ est $(n+1)$-tronqué, et que $\pi_{n+1}\left(F_{n, q+1}, *\right) \longrightarrow \pi_{n+1}\left(F_{n, q}, *\right)$ est un épimorphisme de schémas en groupes unipotents. Tout d'abord, $\widetilde{F_{n, q}}$ étant la fibre homotopique d'un morphisme $F_{n, q} \longrightarrow K(H, n+2)$, on voit que $\widetilde{F_{n, q}}$ est $(n+1)$-tronqué et que $\pi_{n+1}\left(\widetilde{F_{n, q}}, *\right)$ est une extension de $\pi_{n+1}\left(F_{n, q}, *\right)$ par $H$. Comme $H$ est un schéma en groupes unipotent par construction, on voit par récurrence que $\pi_{n+1}\left(\widetilde{F_{n, q}}, *\right)$ est un encore un schéma en groupes unipotent. Ceci montre aussi que $\tau_{\leq n} \widehat{F_{n, q}} \simeq F_{n}$. De même, on montre que $F_{n, q+1}$ est $(n+1)$-tronqué, que $\tau_{\leq n} F_{n, q+1} \simeq F_{n}$, et que $\pi_{n+1}\left(F_{n, q+1}, *\right)$ est un schéma en groupes unipotent qui se surjecte sur $\pi_{n+1}\left(F_{n, q}, *\right)$.

Enfin, on voit d'après le lemme 2.4.2 que la fibre homotopique de $F_{n+1} \longrightarrow F_{n}$ est de la forme $\operatorname{Holim}_{q} K\left(\pi_{n+1}\left(F_{n, q}, *\right), n+1\right) \simeq K\left(\operatorname{Lim}_{q} \pi_{n+1}\left(F_{n, q}, *\right), n+1\right)$. Ainsi, le faisceau $\pi_{n+1}\left(F_{n+1}, *\right)$ est une $\mathbb{U}$-limite projective de schéma en groupes 
unipotent et donc est lui-même un schéma en groupes unipotent. Ceci montre au passage que $F_{n+1}$ est $(n+1)$-tronqué, et que $\tau_{\leq n} F_{n+1} \simeq F_{n}$, et termine donc la preuve du théorème 2.4.5.

On peut maintenant rassembler les deux principaux résultats de ce paragraphe en le corollaire suivant.

Corollaire 2.4.10. Le foncteur $\mathbb{R} S p e c: H o\left(k-A l g^{\Delta} / k\right) \longrightarrow H o\left(S P r(k)_{*}\right)$ induit une équivalence entre la sous-catégorie pleine des $k$-algèbres co-simpliciales augmentées appartenant à $\mathbb{U}$ et cohomologiquement connexes, et la sous-catégorie pleine des champs pointés et connexes dont les faisceaux d'homotopie sont représentables par des schémas en groupes affines unipotents.

Un autre corollaire important du théorème 2.4.5 est la description du groupe fondamental d'une affination d'un ensemble simplicial.

Corollaire 2.4.11. Soit $X$ un ensemble simplicial pointé et connexe appartenant à $\mathbb{U}$. Alors il existe un isomorphisme naturel

$$
\mathcal{U}\left(\pi_{1}(X, x)\right) \simeq \pi_{1}\left((X \otimes k)^{u n i}, x\right) .
$$

Preuve: Nous savons d'après le théorème 2.4.5 que $\pi_{1}\left((X \otimes k)^{u n i}, x\right)$ est un schéma en groupes affine et unipotent. De plus, d'après le théorème 2.4.1 et la propriété universelle des affinations, on a pour tout schéma en groupes affine et unipotent $H$

$$
\begin{gathered}
\operatorname{Hom}\left(\pi_{1}(X, x), H\right) \simeq \pi_{0} \underline{\mathbb{H o m}}_{*}(X, K(H, 1)) \simeq \pi_{0} \mathbb{R} \underline{\operatorname{Hom}}_{*}\left((X \otimes k)^{\text {uni }}, K(H, 1)\right) \\
\simeq \operatorname{Hom}\left(\pi_{1}\left((X \otimes k)^{\text {uni }}, x\right), H\right) .
\end{gathered}
$$

On conclut alors d'après la propriété universelle de $\mathcal{U}\left(\pi_{1}(X, x)\right)$.

\subsection{Comparaison avec l'homotopie rationnelle et $p$-adique}

Les résultats de comparaison que nous présentons ici sont le théorème 2.5.1 et ses corollaires 2.5.4 et 2.5.5. A vrai dire, il ne s'agit pas à proprement parler de comparer rigoureusement nos conctructions à celles pré-existantes, mais plutôt de démontrer des analogues des résultats standards. Cette comparaison montrera que les champs affines donnent une solution au problème de la schématisation tel qu'il est énoncé dans l'appendice A.

Le théorème suivant est l'analogue des principaux résultats en homotopie rationnelle et $p$-adique (voir $[41,56,4,16,37]$ ). Nous nous restreindrons aux ensembles simpliciaux nilpotents et de type fini (sur $\mathbb{Z}$ ). Pour nous, il s'agira des $\mathbb{U}$-ensembles simpliciaux pointés $X$, possédant une décomposition de Postnikov 
$\left\{\tau_{\leq n} X\right\}_{n}$, telle que pour tout $n$ il existe une suite finie de diagrammes homotopiquement cartésiens (avec $0 \leq i \leq m(n)$ )

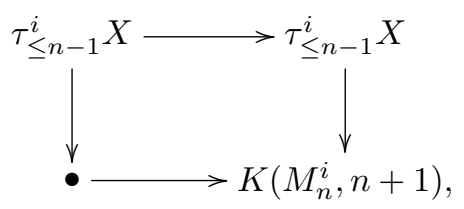

avec $M_{n}$ un groupe abélien de type fini, $\tau_{\leq n-1}^{0} X=\tau_{\leq n-1} X$ et $\tau_{\leq n-1}^{m(n)} X=\tau_{\leq n} X$. Le lecteur pourra s'il le désire adapter luî-même la preuve du théorème suivant pour traiter le cas plus général des espaces nilpotents et de $k$-type finis.

Pour les besoins de l'énoncé rappelons qu'un morphisme d'ensembles simpliciaux $f: X \longrightarrow Y$ est une $k$-équivalence, si les morphismes induits $f^{*}: H^{i}(Y, k) \longrightarrow$ $H^{i}(X, k)$ sont des isomorphismes pour tout $i \geq 0$.

Théorème 2.5.1. $\quad$ - Si $k=\mathbb{Q}$, alors pour tout $\mathbb{U}$-ensemble simplicial pointé, connexe, nilpotent et de type fini $X$, le morphisme d'adjonction

$$
X \longrightarrow \mathbb{R} \Gamma\left((X \otimes k)^{\text {uni }}\right)
$$

est une $\mathbb{Q}$-équivalence.

- Si $k$ est algébriquement clos et de caractéristique $p>0$, alors pour tout $\mathbb{U}$ ensemble simplicial pointé, connexe, nilpotent et de type fini $X$, le morphisme d'adjonction

$$
X \longrightarrow \mathbb{R} \Gamma\left((X \otimes k)^{u n i}\right)
$$

est une $k$-équivalence.

Preuve: D'après la proposition 2.3.2 on sait que $(X \otimes k)^{u n i} \simeq \mathbb{R} S p e c\left(k^{X}\right)$. Un argument standard de décomposition de Postnikov et une utilisation du théorème d'Eilenberg-Moore (voir [55]) permettent alors de se ramener au cas élémentaire $X=K(\mathbb{Z}, n)$ (voir par exemple $[4,37])$. Commençons alors par montrer que $(X \otimes$ $k)^{u n i} \simeq K(\mathcal{U}(\mathbb{Z}), n)$, où $\mathcal{U}(\mathbb{Z})$ est le complété unipotent du groupe discret $\mathbb{Z}$.

Pour cela, le diagramme homotopiquement cartésien

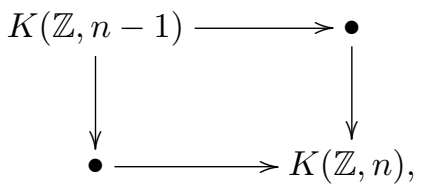

et une nouvelle application du théorème d'Eilenberg-Moore montrent que l'on a

$$
\left.\mathbb{R} \Omega_{*}(K(\mathbb{Z}, n) \otimes k)^{u n i}\right) \simeq(K(\mathbb{Z}, n-1) \otimes k)^{u n i} .
$$

Or, d'après le théorème 2.4 .5 on sait que $(K(\mathbb{Z}, n) \otimes k)^{u n i}$ est connexe, et donc d'après le théorème 1.4 .3 on a

$$
\left.\left.(K(\mathbb{Z}, n) \otimes k)^{u n i}\right) \simeq B \mathbb{R} \Omega_{*}(K(\mathbb{Z}, n) \otimes k)^{u n i}\right) \simeq B(K(\mathbb{Z}, n-1) \otimes k)^{u n i} .
$$

Ceci permet donc de se ramener au cas où $n=1$. 
Lemme 2.5.2. Le moprhisme naturel $H^{i}\left(B \mathcal{U}(\mathbb{Z}), \mathbb{G}_{a}\right) \longrightarrow H^{i}\left(B \mathbb{Z}, \mathbb{G}_{a}\right)$ est un isomorphisme pour tout $i$.

Preuve: Pour $i=0$ c'est immédiat. Pour $i=1$, on a d'après la propriété universelle de $\mathcal{U}(\mathbb{Z})$

$$
H^{1}\left(B \mathcal{U}(\mathbb{Z}), \mathbb{G}_{a}\right) \simeq \operatorname{Hom}\left(\mathcal{U}(\mathbb{Z}), \mathbb{G}_{a}\right) \simeq k \simeq H^{1}\left(B \mathbb{Z}, \mathbb{G}_{a}\right) .
$$

Enfin, $\mathcal{U}(\mathbb{Z})$ étant un schéma en groupes unipotent libre, on sait que $H^{i}\left(B \mathcal{U}(\mathbb{Z}), \mathbb{G}_{a}\right)$ est trivail pour $i>1$ (voir par exemple la preuve de 3.3.5).

Le lemme précédent montre que le morphisme $K(\mathbb{Z}, 1) \longrightarrow K(\mathcal{U}(\mathbb{Z}), 1)$ est une $\mathcal{O}$-équivalence. Ainsi, comme d'après le théorème 2.4.1 $K(\mathcal{U}(\mathbb{Z}), 1)$ est un champ affine, on a $(K(\mathbb{Z}, 1) \otimes \mathbb{Q})^{\text {uni }} \simeq K(\mathcal{U}(\mathbb{Z}), 1)$. Ceci achève la preuve que $(K(\mathbb{Z}, n) \otimes k)^{u n i} \simeq K(\mathcal{U}(\mathbb{Z}), 1)$. Remarquons que l'on n'a toujours pas utilisé l'hypothèse sur $k$.

Il nous faut encore montrer que le morphisme d'adjonction

$$
K(\mathbb{Z}, n) \longrightarrow \mathbb{R} \Gamma(K(\mathcal{U}(\mathbb{Z}), n))
$$

est une $k$-équivalence. Pour cela remarquons tout d'abord que le faisceau en groupes $\mathcal{U}(\mathbb{Z})$ est acyclique sur $(A f f / k)_{f p q c}$. En effet, si $k$ est de caractéristique nulle on a $\mathcal{U}(\mathbb{Z}) \simeq \mathbb{G}_{a}$ qui est un faisceau quasi-cohérent et donc acyclique. Dans le cas où $k$ est de caractéristique $p$, on a $\mathcal{U}(\mathbb{Z}) \simeq \mathbb{Z}_{p}$, le groupe pro-fini des entiers $p$-adiques (vu comme un schéma pro-algébrique, et donc affine sur $S p e c k$ ). Mais comme alors $k$ est algébriquement clos on sait que

$$
H_{\text {fpqc }}^{i}\left(\text { Speck }, \mathbb{Z}_{p}\right) \simeq H_{\text {et }}^{i}\left(\text { Speck }, \mathbb{Z}_{p}\right) \simeq 0
$$

dès que $i>0$.

On peut donc conclure que lorsque $k=\mathbb{Q}$ on a

$$
\mathbb{R} \Gamma((\mathcal{U}(\mathbb{Z}), n)) \simeq K(\mathbb{Q}, n),
$$

et lorsque $k$ est de caractéristique $p$ et algébriquement clos on a

$$
\mathbb{R} \Gamma((\mathcal{U}(\mathbb{Z}), n)) \simeq K\left(\mathbb{Z}_{p}, n\right) .
$$

Dans les deux cas, il est bien connu que les morphismes d'adjonction

$$
K(\mathbb{Z}, n) \longrightarrow K(\mathbb{Q}, n) \quad K(\mathbb{Z}, n) \longrightarrow K\left(\mathbb{Z}_{p}, n\right)
$$

sont des $k$-équivalences.

Remarque: Le lecteur pourra par exemple comparer la preuve du point (2) du théorème précédent avec la preuve du théorème principal de [37], où le point crucial est de montrer que $K(\mathbb{Z} / p, n)$ est $k$-résolvable (voir $[37,4]$ ). Le fait que $K(\mathbb{Z} / p, n)$ soit $k$-résolvable est en réalité tout à fait analogue à la condition que $K(\mathbb{Z} / p, n)$ soit un champ affine, qui dans notre cas est une conséquence directe de la théorie. Il est par ailleurs intéressant de constater que la construction du modèle explicite pour la $E_{\infty}$-algèbre $C^{*}(K(\mathbb{Z} / p, n), k)$ qui est présentée dans [37, 4], est 
très proche de la construction qui exibe le champ $K(\mathbb{Z} / p, n)$ comme la fibre homotopique du morphisme $1-\mathbf{F}: K\left(\mathbb{G}_{a}, n\right) \longrightarrow K\left(\mathbb{G}_{a}, n\right)$, où $\mathbf{F}$ est l'endomorphisme de $\mathbb{G}_{a}$ qui élève à la puissance $p$.

Le corollaire suivant est un corollaire important de la démonstration du théorème précédent.

Corollaire 2.5.3. Soit $X$ un $\mathbb{U}$-ensemble simplicial pointé, connexe, nilpotent et de type fini. Alors, pour tout $i>0$, le faisceau $\pi_{i}\left((X \otimes k)^{\text {uni }}, x\right)$ est représentable par le complété unipotent du groupe $\pi_{i}(X, x)$. En particulier, pour $i>1$ on a

$$
\pi_{i}\left((X \otimes k)^{u n i}, x\right) \simeq \begin{cases}\pi_{i}(X, x) \otimes_{\mathbb{Z}} \mathbb{G}_{a} & \text { si car } k=0 \\ \pi_{i}(X, x) \otimes_{\mathbb{Z}} \mathbb{Z}_{p} & \text { si car } k=p .\end{cases}
$$

Le $(-\otimes k)^{u n i}$ envoient clairement les $k$-équivalences sur des isomorphismes dans $\operatorname{Ho}\left(\operatorname{SPr}(k)_{*}\right)$. Il se factorise donc en un foncteur

$$
(-\otimes k)^{u n i}: H o_{k}\left(\mathbb{U}-S E n s_{0}\right) \longrightarrow H o\left(\operatorname{SPr}(k)_{*}\right),
$$

où $H o_{k}\left(\mathbb{U}-S E n s_{0}\right)$ est la catégorie localisée de $H o\left(\mathbb{U}-S E n s_{0}\right)$ le long le long des $k$-équivalences.

Corollaire 2.5.4. Lorsque $k$ est ou bien le corps des rationnels $\mathbb{Q}$, ou bien algébriquement clos et de caractéristique $p>0$, le foncteur

$$
(-\otimes k)^{u n i}: H o_{k}\left(\mathbb{U}-S E n s_{0}\right) \longrightarrow H o\left(S P r(k)_{*}\right)
$$

restreint à la sous-catégorie pleine des ensembles simpliciaux nilpotents et de type fini, est pleinement fidèle.

Preuve: C'est immédiat d'après le théorème 2.5.1.

C'est le corollaire précédent qui permet d'affirmer que le champ $(X \otimes k)^{\text {uni }}$ est un modèle pour l'homotopie rationnel ou bien $p$-adique, suivant que $k=\mathbb{Q}$ ou bien $k=\overline{\mathbb{F}}_{p}$.

Corollaire 2.5.5. Soit $X$ et $Y$ deux $\mathbb{U}$-ensembles simpliciaux pointés, connexes, nilpotents et de type fini, et supposons que $k$ soit de caractéristique $p>0$. Si $(X \otimes k)^{u n i}$ et $(Y \otimes k)^{\text {uni }}$ sont isomorphes dans $H o\left(\operatorname{SPr}(k)_{*}\right)$, alors $X$ et $Y$ sont isomorphes dans $H_{k}\left(\mathbb{U}-S E n s_{0}\right)$.

Preuve: En effet, si $\bar{k}$ est une clôture algébrique de $k$, alors d'après le corollaire 2.3.5 $(X \otimes \bar{k})^{u n i} \simeq(Y \otimes \bar{k})^{u n i}$. Cet isomorphisme induit d'après le théorème 2.5.1 un isomorphisme entre $X$ et $Y$ dans $H o_{\bar{k}}\left(\mathbb{U}-S E n s_{0}\right)$. Or, il est facile de vérifier qu'un morphisme est une $\bar{k}$-équivalence si et seulement si c'est une $k$ équivalence. Ainsi, il existe une équivalence de catégories entre $H o_{\bar{k}}\left(\mathbb{U}-S E n s_{0}\right)$ et $H o_{k}\left(\mathbb{U}-S E n s_{0}\right)$. Les ensembles simpliciaux $X$ et $Y$ sont donc isomorphes dans le catégorie $H o_{k}\left(\mathbb{U}-S E n s_{0}\right)$. 


\subsection{Critique des champs affines}

Pour terminer ce chapitre nous souhaitons faire la critique des champs affines et du procédé d'affination des types d'homotopie. Nous avons vus que les champs affines sont tous construit à partir d'extensions successives de champs de la forme $K\left(\mathbb{G}_{a}, n\right)$. Ainsi, d'un point de vue de la théorie de l'homotopie les champs affines sont des types d'homotopie pro-nilpotents. Ceci implique en particulier que le foncteur d'affination tue toute l'information non nilpotente que peut posséder un type d'homotopie. Par exemple, si $X$ est un type d'homotopie tel que son groupe fondamental opère de façon semi-simple sur ses groupes d'homotopie supérieur, le champ $(X \otimes \mathbb{Z})^{u n i}$ ne verra pas une partie importante de l'information homotopique que contient $X$. A titre d'exemple, examinons le cas très simple suivant.

Soit $X=K\left(\mathbb{Z} / m, \mathbb{Z}^{r}, n\right)$, où $\mathbb{Z} / m$ opère non trivialement sur le groupe abélien $\mathbb{Z}^{r}$, et supposons que $k=\mathbb{Q}$. Cette action s'étend en une action de $\mathbb{Z} / m$ sur le schéma en groupes affine $\mathbb{G}_{a}^{r}$, et donne une décomposition $\mathbb{G}_{a}^{r} \simeq \mathbb{G}_{a}^{i} \times \mathbb{G}_{a}^{r-i}$, où le premier facteur est le sous-groupe des points fixes. On peut alors vérifier que

$$
\pi_{1}\left((X \otimes \mathbb{Q})^{u n i}, *\right) \simeq 0 \quad \pi_{n}\left((X \otimes \mathbb{Q})^{u n i}, *\right) \simeq \mathbb{G}_{a}^{i},
$$

Ainsi, la partie non triviale de l'action de $\mathbb{Z} / m$ sur $\pi_{n}(X)$ disparait dans le champ $(X \otimes \mathbb{Q})^{\text {uni }}$.

De façon beaucoup plus générale, toute l'information concernant les représentations non-nilpotentes des groupes fondamentaux dans les groupes d'homotopie supérieurs sera inaccessible à l'aide de la construction $(-\otimes \mathbb{Z})^{\text {uni }}$.

L'idée naturelle pour remédier à ce problème est d'élargir la définition de champs affines afin de permettre tous les schémas en groupes affines comme des groupes fondamentaux potentiels (ce point de vue est bien entendu relié à la dualité de Tannaka). C'est dans cette optique que nous introduirons les $\infty$-gerbes affines et les types d'homotopie schématiques dans le chapitre suivant.

\section{3. $\infty$-Gerbes affines et types d'homotopie schématiques}

Dans ce dernier chapitre nous introduisons la seconde notion fondamentale de ce travail, celle d' $\infty$-gerbe affine et de type d'homotopie schématique. L'idée est d'utiliser la notion de champ affine afin de donner une version homotopique de la notion de gerbe affine utilisée dans le formalisme Tannakian (voir [43, 10]). Pour cela, rappelons qu'une gerbe affine neutralisée peut s'interpréter comme un champ de la forme $K(G, 1)$, où $G$ est un schéma en groupes affine et plat sur l'anneau de base $k$. On pourrait aussi dire qu'il s'agit d'un champ pointé et connexe dont le champ des lacets est représentable par un schéma affine et plat. Ce point de vue nous incite tout naturellement à définir une $\infty$-gerbe affine pointée comme un champ pointé et connexe dont le champ des lacets est un champ affine et plat sur $k$. C'est la définition que nous voulons adopter. Cependant, pour des raisons 
techniques nous ne voulons pas définir la notion de platitude requise, et nous nous restreindrons donc au cas où l'anneau de base est un corps.

D'un point de vue technique, le fait que les champs affines soient des objets $\mathcal{O}$-locaux est fondamental pour que la notion d'affination définie dans 2.3.1 reste raisonnable (i.e. possède une propriété d'unicité et de fonctorialité). De même, pour pouvoir par la suite définir une notion raisonnable de schématisation, il est à prévoir que les types d'homotopie schématiques devront satisfaire à une certaine condition de localité pour une théorie cohomologique convenable. C'est pour cela que nous introduirons les notions de $P$-équivalence et d'objets $P$-locaux, qui généralisent les notions de $\mathcal{O}$-équivalences et d'objets $\mathcal{O}$-locaux en ce sens qu'elles concernent la cohomologie à valeurs dans tous les systèmes locaux de $k$-espaces vectoriels. Nous définirons alors les types d'homotopie schématiques comme les $\infty$-gerbes affines pointées qui sont de plus des objets $P$-locaux. Cependant, nous conjecturons que toute $\infty$-gerbe affine pointée est un champ $P$-local, et donc que les types d'homotopie schématiques pointés coincident avec les $\infty$-gerbes affines pointées (voir 3.2.10).

Une fois ces notions définies nous définirons la notion de schématisation d'un type d'homotopie, et nous montrerons que tout type d'homotopie dans $\mathbb{U}$ possède une schématisation. Nous montrerons alors à travers quelques exemples en quoi le foncteur de schématisation diffère de celui d'affination, et en quoi il résoud le problème soulevé au cours de paragraphe 2.6.

Pour tout ce chapitre $k$ est un corps de caractéristique quelconque.

\subsection{Définitions}

Dans ce paragraphe nous donnerons la définition d' $\infty$-gerbe affine, et de type d'homotopie schématique. Nous n'étudierons essentiellement aucune de leurs propriétés, et nous nous contenterons de donner une méthode permettant de construire des exemples à partir de schémas en groupes affines simpliciaux. Celle-ci sera utilisée dans par la suite pour construire le foncteur de schématisation.

Le lecteur remarquera que nous ne donnerons la définition de type d'homotopie schématique que lorsque $k$ est un corps. De plus, la conjecture 3.2.10 prévoit que les types d'homotopie schématiques pointés sont exactement les $\infty$-gerbes affines pointés. Cependant, comme un tel résultat n'est certainement pas vraie sur une base générale (pour une généralisation adéquate des notions d' $\infty$-gerbes affines et de types d'homotopie schématiques), faire la différence entre les $\infty$-gerbes affines pointées et les types d'homotopie schématiques pointés me semble raisonnable.

Pour commencer nous allons définir la notion de $P$-équivalence entre préfaisceaux simpliciaux pointés, qui est une notion plus forte que celle de $\mathcal{O}$-équivalence car faisant aussi intervenir les systèmes locaux de $k$-espaces vectoriels de dimension finie.

Définition 3.1.1. - Un morphisme entre préfaisceaux simpliciaux pointés et connexes $f: G \longrightarrow H$ est une $P$-équivalence, si pour tout schéma en groupes 
affine et de type fini $K$, toute représentation linéaire de dimension finie $\mathbb{V}$ de $K$, et tout entier $n>0$, le morphisme induit

$$
f^{*}: \mathbb{R} \underline{\operatorname{Hom}}_{*}(H, K(K, V, n)) \longrightarrow \mathbb{R} \underline{\operatorname{Hom}}_{*}(G, K(K, V, n))
$$

est un isomorphisme dans $H o(\mathbb{V}-S E n s)$.

- Un préfaisceau simplicial pointé et connexe $F$ est dit $P$-local si pour toute $P$-équivalence $f: G \longrightarrow H$, le morphisme induit

$$
f^{*}: \mathbb{R} \underline{\operatorname{Hom}}_{*}(H, F) \longrightarrow \mathbb{R} \underline{\operatorname{Hom}}_{*}(G, F)
$$

est un isomorphisme dans $H o(\mathbb{V}-S E n s)$.

On remarquera qu'un morphisme de préfaisceaux simpliciaux pointés et connexes qui est une $\mathcal{O}$-équivalence est aussi une $P$-équivalence.

Remarque: Nous avons choisi l'expression $P$-équivalence par référence aux complexes parfaits. En effet, on peut montrer qu'un morphisme est une $P$-équivalence si et seulement si c'est une équivalence pour la théorie cohomologique non-abélienne déterminée par le 1-champ de Segal des complexes parfaits (voir $[23, \S 21])$. C'est ce point de vue plus général qui devra être utilisé pour donner une définition raisonnable des types d'homotopie schématiques sur des bases plus générales.

Rappelons que pour un préfaisceau simplicial $* \longrightarrow F$, on peut définir son préfaisceau simplicial des lacets $\Omega_{*} F$. Le foncteur $\Omega_{*}$ étant alors un foncteur de Quillen à droite de la catégorie des préfaisceaux simpliciaux pointés dans elle même, nous noterons $\mathbb{R} \Omega_{*}$ son foncteur dérivé à droite.

Définition 3.1.2. - Un préfaisceau simplicial pointé $s: * \longrightarrow F$ est une $\infty$-gerbe affine pointée sur $k$ s'il vérifie les deux conditions suivantes.

- Le préfaisceau simplicial $F$ est connexe (i.e. $\pi_{0}(F) \simeq *$ ).

- Le préfaisceau simplicial $\mathbb{R} \Omega_{*} F$ est un champ affine sur $k$.

- Un type d'homotopie schématique pointé est une $\infty$-gerbe affine pointée qui est de plus $P$-local (en tant que préfaisceau simplicial pointé).

Remarques:

- Par définition une $\infty$-gerbe affine pointée est un préfaisceau simplicial pointé et sera donc toujours considérée comme un objet dans $S \operatorname{Pr}(k)_{*}$ ou dans $H o\left(S \operatorname{Pr}(k)_{*}\right)$. Ainsi, un morphisme entre deux tels objets sera toujours un morphisme d'objets pointés.

- Soit $F=K(G, 1)$ une gerbe neutre sur Speck, liée par un schéma en groupes affine $G$ (voir $[43,10]$ ). Alors, vu comme préfaisceau simplicial $F$ est une $\infty$-gerbe affine (voir [29] pour le passage des champs en groupoïdes aux préfaisceaux simpliciaux). Ainsi, tout comme la notion de champ affine est une généralisation de celle de schéma affine, la notion d' $\infty$-gerbe affine généralise celle de gerbe affine neutre. Il faut donc penser à la théorie des gerbes 
affines neutres comme à la théorie homotopique schématique 1-tronquée (et connexe).

- Nous ne définirons pas la notion de type d'homotopie schématiques sur un anneau $k$ quelconque, la notion de $P$-équivalences dans ce cas passant par l'existence du 1-champ de Segal des complexes parfaits que nous ne souhaitons pas définir ici (voir cependant [23]).

Il est important de noter le fait élémentaire suivant.

Lemme 3.1.3. Toute $\infty$-gerbe affine pointée est un champ sous-affine.

Preuve: Considérons un remplacement cofibrant dans $\operatorname{Pr}-\Delta^{o}-\operatorname{SPr}(k)$, $H \longrightarrow \mathbb{R} \Omega_{*} F$, ainsi que l'objet co-simplicial de $k-A l g^{\Delta}$

$$
\begin{array}{rlll}
\mathcal{O}\left(H_{\bullet}\right): & \Delta^{o} & \longrightarrow k-A l g^{\Delta} \\
& {[n]} & \mapsto \mathcal{O}\left(H_{n}\right) .
\end{array}
$$

Soit $A^{(\bullet)} \longrightarrow \mathcal{O}\left(H_{\bullet}\right)$ un remplacement cofibrant de ce diagramme, avec $A^{(0)}=$ $H_{0}=k$. Alors, comme chaque $H_{n}$ est un champ affine (car équivalent à $\mathbb{R} \Omega_{*}(F)^{n}$ ), le morphisme d'adjonction

$$
\mathbb{R} \Omega_{*} F \longrightarrow \operatorname{Spec} A^{(\bullet)}
$$

est un isomorphisme dans $H o\left(\operatorname{Pr}-\Delta^{o}-S \operatorname{Pr}(k)\right)$. En passant aux classifiants, on trouve un isomorphisme dans $\operatorname{Ho}\left(\operatorname{SPr}(k)_{*}\right)$

$$
F \simeq B \mathbb{R} \Omega_{*} F \simeq B \operatorname{Spec} A^{(\bullet)} .
$$

Comme le préfaisceau simplicial $B \operatorname{Spec} A^{(\bullet)}$ est représenté par le schéma affine simplicial $[n] \mapsto \operatorname{Spec} A_{n}^{(n)}, F$ est bien un champ sous-affine.

Une $\infty$-gerbe affine pointée $F$ est la même chose qu'un $H_{\infty}$-champ dont le champ sous-jacent est un champ affine. Comme la notion de $H_{\infty}$-champ est une notion diagrammatique, on en déduit une notion duale au niveau des $k$-algèbres co-simpliciales. Ce n'est pas une notion que nous utiliserons, mais nous donnerons sa définition à titre indicatif.

Définition 3.1.4. Une $H_{\infty}$-algèbre de Hopf sur $k$ est la donnée d'un foncteur

$$
A^{(\bullet)}: \Delta \longrightarrow k-A l g^{\Delta}
$$

tel que les trois propriétés suivantes soient satisfaites.

- $A^{(0)}=k$

- Pour tout $n>0$, le morphisme naturel

$$
\underbrace{A^{(1)} \otimes_{k}^{\mathbb{L}} A^{(1)} \otimes_{k}^{\mathbb{L}} \cdots \otimes_{k}^{\mathbb{L}} A^{(1)}}_{n \text { fois }} \rightarrow A^{(n)}
$$

est un isomorphisme dans $H o\left(k-A l g^{\Delta}\right)$.

- Vue dans la catégorie homotopique $H o\left(k-A l g^{\Delta}\right)$, la loi naturelle de comonoïde induite sur $A_{1}$ est une loie de co-groupe. 
Bien entendu, il existe une équivalence de la catégorie homotopique des $H_{\infty}$ algèbres de Hopf sur $k$ et appartenant à $\mathbb{U}$ et de la catégorie des $\infty$-gerbes affines pointées sur $k$. Cette équivalence ce déduit immédiatement du corollaire 2.2.3.

Nous terminerons ce chapitre par un procédé général de construction d' $\infty$ gerbes affines. Il permet de donner de très nombreux exemples, mais nous ne savons pas s'ils sont tous obtenus de cette façon. Nous montrerons par la suite que cette construction fournit en réalité des types d'homotopie schématiques, ce qui nous permettra de construire le foncteur de schématisation.

Considérons un schéma en groupes affine simplicial $G$, et notons encore $G \in$ $G p S \operatorname{Pr}(k)$ le préfaisceau en groupes simplicial qu'il représente. La loi de groupe $\mu: G \times G \longrightarrow G$ induit par fonctorialité une co-multiplication

$$
\mu^{*}: \mathcal{O}(G) \longrightarrow \mathcal{O}(G \times G) \simeq \mathcal{O}(G) \otimes \mathcal{O}(G),
$$

qui fait de $\mathcal{O}(G)$ une $k$-algèbre de Hopf commutative co-simpliciale.

On peut alors montrer que $\mathcal{O}(G) \otimes \mathcal{O}(G)$ est isomorphe dans $H o\left(k-A l g^{\Delta}\right)$ au produit tensoriel dérivé $\mathcal{O}(G) \otimes^{\mathbb{L}} \mathcal{O}(G)$. En particulier, ceci montre que le morphisme naturel

$$
\mathbb{R} \operatorname{Spec} \mathcal{O}(G \times G) \longrightarrow \mathbb{R} \operatorname{Spec} \mathcal{O}(G) \times \mathbb{R} \operatorname{Spec} \mathcal{O}(G)
$$

est une équivalence. Plus généralement, ceci implique que $\mathcal{O}(G)$, vu comme objet dans la catégorie homotopique $H o\left(k-A l g^{\Delta}\right)$ est un objet en co-groupes (i.e. un objet en groupes dans la catégorie opposée). Le diagramme simplicial

$$
\begin{array}{cccc}
\mathbb{R} \operatorname{Spec} \mathcal{O}(G): & \Delta^{o} & \longrightarrow & \operatorname{SPr}(k) \\
& {[n]} & \mapsto & \mathbb{R} \operatorname{Spec} \mathcal{O}\left(G^{n}\right),
\end{array}
$$

est donc un $H_{\infty}$-champ au sens de la définition 1.4.2, que nous noterons symboliquement $\mathbb{R} \operatorname{Spec} \mathbb{L} \mathcal{O}(G)$. De façon duale, on peut aussi affirmer que le diagramme co-simplicial

$$
\begin{array}{llll}
\mathbb{L} \mathcal{O}(G): & \Delta^{o} & \longrightarrow & k-A l g^{\Delta} \\
{[n]} & \mapsto & \mathbb{L} \mathcal{O}\left(G^{n}\right),
\end{array}
$$

est une $H_{\infty}$-algèbre de Hopf (voir Def. 3.1.4).

Le théorème 1.4.3 implique alors que $F=B \mathbb{R} \operatorname{Spec} \mathbb{L} \mathcal{O}(G)$ est une $\infty$-gerbe affine pointée, avec $\mathbb{R} \Omega_{*}(F) \simeq \mathbb{R} \operatorname{Spec} \mathcal{O}(G)$. Remarquer de plus qu'il existe un morphisme naturel dans $H o\left(S \operatorname{Pr}(k)_{*}\right)$ induit par l'adjonction entre les foncteurs $\mathcal{O}$ et $S p e c$

$$
B G \longrightarrow F
$$

L'intérêt de cette construction est qu'elle donne un objet possédant une propriété universelle.

Lemme 3.1.5. Sous les hypothèses précédentes, le morphisme naturel

$$
B G \longrightarrow F=B \mathbb{R} \operatorname{Spec} \mathcal{O}(G)
$$


est universel dans $H o\left(S \operatorname{Pr}(k)_{*}\right)$ pour les morphismes de $B G$ vers des $\infty$-gerbes affines pointées.

Preuve: Par définition des $\infty$-gerbes affines pointées il suffit de montrer que le morphisme d'adjonction $G \longrightarrow \mathbb{R} \operatorname{Spec} \mathcal{O}(G)$, vu comme un morphisme dans $H o\left(\operatorname{Pr}-\Delta^{o}-S \operatorname{Pr}(k)\right)$, est universel vers les $H_{\infty}$-champs dont le champ sousjacent est affine. Mais, ceci n'est qu'une version en famille du corollaire 2.2.3.

Remarque: La question de savoir si toutes les $\infty$-gerbes affines pointées sont obtenues par cette construction est en fait équivalente à un problème de strictification des $H_{\infty}$-algèbres de Hopf en algèbre de Hopf co-simpliciales.

\subsection{Exemples d' $\infty$-gerbes affines et de types d'homotopie schématiques}

Etant donnée leurs définitions il est assez difficile de produire des exemples explicites de $\infty$-gerbes affines. Cela provient principalement du fait que l'on connait assez mal leurs faisceaux d'homotopie en général. Pour remédier à cela nous allons démontrer un critère de reconnaissance des types d'homotopie schématiques analogues au théorème 2.4.1. Ceci permet de donner de très nombreux exemples de types d'homotopie schématiques, dont en particulier les champs très présentables introduits par C. Simpson (voir [51] et [52]).

Nous commençerons par citer deux lemmes utiles pour construire des types d'homotopie schématiques.

Lemme 3.2.1. Tout champ affine pointé et connexe est un type d'homotopie schématique pointé.

Preuve: Soit $A$ une $k$-algèbre co-simpliciale de $\mathbb{U}$ telle que $F$ soit équivalent à $\mathbb{R} S$ pec $A$, et $A \longrightarrow k$ l'augmentation correspondant au point de $F$. Il est alors facile de voir qu'il existe un isomorphisme dans la catégorie homotopique $\operatorname{Ho}(\operatorname{SPr}(k))$

$$
\mathbb{R} \Omega_{*} F \simeq \mathbb{R} S \operatorname{Spec}\left(k \otimes_{A}^{\mathbb{L}} k\right) .
$$

Comme $k$ est corps ceci montre bien que $\mathbb{R} \Omega_{*} F$ est un champ affine, et donc que $F$ est une $\infty$-gerbe affine. De plus, il est immédiat qu'un objet $\mathcal{O}$-local est aussi un objet $P$-local (car une $P$-équivalence est une $\mathcal{O}$-équivalence). Par définition, $F$ est bien un type d'homotopie schématique pointé.

Lemme 3.2.2. Tout champ pointé et connexe qui est une $\mathbb{U}$-limites homotopique de types d'homotopie schématiques pointés et lui-même un type d'homotopie schématique pointé et connexe.

Proof: En appliquant 2.2.7 aux champs des lacets on montre que le lemme est vrai pour les $\infty$-gerbes affines pointées. De plus, il est clair que les objets $P$-locaux sont stables par $\mathbb{U}$-limites homotopiques. 
Lemme 3.2.3. Soit $F$ un préfaisceau simplicial pointé et connexe, et supposons que $\mathbb{R} \Omega_{*} F$ soit sous-affine et que $F$ soit une $\mathbb{V}$-limite homotopique (dans la catégorie des préfaisceaux simpliciaux pointés) de types d'homotopie schématiques pointés. Alors $F$ est un type d'homotopie schématique pointé.

Preuve: Comme $k$ est un corps tout les champs affines sont plats sur $k$. De plus, le foncteur $\mathbb{R} \Omega_{*}$ étant de Quillen à droite il préserve les limites homotopiques. Le lemme est donc une conséquence du corollaire 2.2.11, et du fait que les objets $P$-locaux sont stables par $\mathbb{V}$-limites homotopiques.

Le théorème suivant est un analogue du théorème 2.4.1.

Théorème 3.2.4. Soit $* \longrightarrow F$ un préfaisceau simplicial pointé et connexe. Supposons que les deux assertions suivantes soient satisfaites.

- Le faisceau en groupes $\pi_{1}(F, *)$ est représenté par un schéma en groupe affine.

- Pour tout entier $i>1$ le faisceau en groupes $\pi_{i}(F, *)$ est représenté par un schéma en groupes affine et unipotent.

Alors $F$ est est un type d'homotopie schématique pointé.

Preuve: Commençons par montrer que $\mathbb{R} \Omega_{*} F$ est affine (i.e. que $F$ est une $\infty$-gerbe affine pointée).

Le même argument que celui utilisé dans la preuve du théorème 2.4.1 montre que $F$ satisfait aux hypothèses de la proposition 1.2.2. Un argument de décomposition de Postnikov et une utilisation du lemme 3.2.2 montre alors qu'il suffit de traiter le cas élémentaire où $F=K(G, M, n)$, avec $G$ un schéma en groupes affine, et $M$ un schéma en groupe affine et unipotent. Mais dans ce cas on a une équivalence de préfaisceaux simpliciaux (mais pas de $H_{\infty}$-champs) $\mathbb{R} \Omega_{*} K(G, M, n) \simeq G \times K(M, n-1)$. Comme le faisceau $G$ est représenté par un schéma affine c'est un champ affine. De plus, $M$ étant un schéma en groupes affine et unipotent on sait d'après le théorème 2.4.1 que $K(M, n)$ est un champ affine. Le champ $F$ est ainsi un produit de champs affines, et est donc lui-même affine.

Enfin, pour voir que $F$ est $P$-local, on utilise que les champs $P$-locaux sont stables par les $\mathbb{V}$-limites homotopiques. En utilisant le même argument que pour la preuve du théorème 2.4 .1 on peut supposer que $F$ est de la forme $K(G, M, n)$, avec $G$ et $M$ de type fini sur $k$. On applique alors les mêmes technique que lors de la preuve du théorème 2.2.9 (en particulier les lemmes 2.4.2 et 2.4.3) pour se ramener au cas où $M$ est une représentation linéaire de dimension finie de $G$. Mais dans ce cas, $K(G, M, n)$ est tautologiquement $P$-local.

La définition suivante est une adapation de la définition de champs très présentables de $[51,52]$. Elle diffère un peu de la définition originale dans le sens où l'on ne considère que des préfaisceaux simpliciaux pointés et connexes, et où l'on ne se restreint pas à la caractéristique nulle.

Définition 3.2.5. Un champ très présentable (pointé et connexe) est un préfaisceau simplicial pointé et connexe $* \longrightarrow F$, satisfaisant les trois conditions suivantes. 
- Il existe un entier $n$ tel que le préfaisceau simplicial $F$ soit $n$-tronqué (i.e. $F$ est équivalent à $\left.\tau_{\leq n} F\right)$.

- Le faisceau en groupes $\pi_{1}(F, *)$ est représenté par un schéma en groupes affine et de type fini sur $k$.

- Pour tout entier $i>0$ le faisceau en groupes $\pi_{i}(F, *)$ est représenté par un schéma en groupes affine unipotent et de type fini sur $k$.

Par définition un champ très présentable est un objet pointé et sera donc toujours considéré comme un objet dans $\operatorname{SPr}(k)_{*}$.

On tire immédiatement du théorème 3.2.4 le corollaire important suivant.

Corollaire 3.2.6. Tout champ très présentable est un type d'homotopie schématique pointé.

Le théorème 3.2.4 permet de démontrer le corollaire suivant. Pour cela, rappelons qu'un paragraphe précédent nous avons associé à tout schéma en groupes affine simplicial $G$ une $\infty$-gerbe affine $B \mathbb{R} \operatorname{Spec} \mathcal{O}(G)$.

Corollaire 3.2.7. Soit $G$ un schéma en groupes affine simplicial et considérons $F:=B \mathbb{R} \operatorname{Spec} \mathcal{O}(G)$ l'œ-gerbe affine pointée qui lui est associée. Alors, le faisceau $\pi_{i}(F, *)$ est représentable par un schéma en groupes affine, qui est de plus unipotent dès que $i>1$.

En particulier, pour tout schéma en groupes affine simplicial $G$, le champ pointé $B \mathbb{R} S \operatorname{pec} \mathcal{O}(G)$ est un type d'homotopie schématique pointé.

Preuve: Notons $A:=\mathbb{L} \mathcal{O}\left(\Omega_{*} F\right) \simeq \mathcal{O}(G)$ la $k$-algèbre co-simpliciale de cohomologie de $\Omega_{*} F \simeq G$. Comme $F$ est une $\infty$-gerbe affine, on sait que $A$ est une $H_{\infty}$-algèbre de Hopf (voir Def. 3.1.4). Ceci implique en particulier que $H^{*}(A)$ est une $k$-algèbre de Hopf graduée.

Notons $B$ la $k$-algèbre co-simpliciale définie par le diagramme homotopiquement co-cartésien suivant

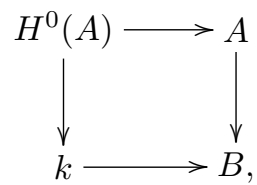

où $H^{0}(A) \longrightarrow A$ est le morphisme naturel, et $H^{0}(A) \longrightarrow k$ l'augmentation correspondant à la co-unité de $H^{*}(A)$.

Lemme 3.2.8. Pour tout entier $n, H^{n}(A)$ est un $H^{0}(A)$-module libre. De plus, on a $H^{n}(B) \simeq H^{n}(A) \otimes_{H^{0}(A)} k$.

Preuve: La seconde assertion est clairement impliquée par la première.

Notons $G=\operatorname{Spec} H^{0}(A)$ le schéma en groupes affine correspondant à la $k$ algèbre de Hopf $H^{0}(A)$. Pour tout $n, H^{n}(A)$ est naturellement un $H^{0}(A)$-module, et correspond à un faisceau quasi-cohérent sur $G$, dont on cherche à montrer qu'il 
est libre. La structure d'algèbre de Hopf induit sur $H^{n}(A)$ une structure de faisceau quasi-cohérent $G$-équivariant sur $G$ (pour l'action de $G$ sur lui-même par translations). Ainsi, le faisceau quasi-cohérent $H^{n}(A)$ est trivial sur $G$ (i.e. de la forme $V \otimes_{k} \mathcal{O}_{G}$, où $V$ est un $k$-espace vectoriel), et donc $H^{n}(A)$ est un $H^{0}(A)$ module libre.

Considérons le diagramme homotopiquement cartésien de champs

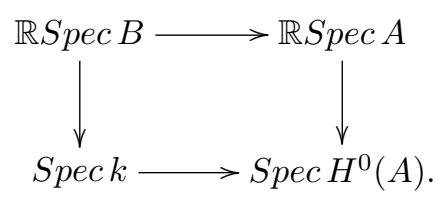

Comme le lemme précédent montre que $B$ est une $k$-algèbre co-simpliciale augmentée et connexe, le théorème 2.4 .5 et la suite exacte longue en homotopie impliquent que les faisceaux $\pi_{i}(F, *)$ sont des schémas en groupes unipotents pour $i>1$. De plus, le morphisme $\pi_{1}(F, *) \longrightarrow S p e c H^{0}(A)$ est alors un monomorphisme. Il nous reste donc à montrer que c'est un épimorphisme de faisceaux.

Pour cela on considère les morphismes naturels

$$
G \longrightarrow \mathbb{R} \operatorname{Spec} A \longrightarrow \operatorname{Spec} H^{0}(A) \text {. }
$$

Par construction $\operatorname{Spec} H^{0}(A) \simeq \pi_{0}(G)$ est le schéma en groupes équaliseur des morphismes $d_{1}, d_{0}: G_{1} \longrightarrow G_{0}$. Ainsi, $G_{0} \longrightarrow S p e c H^{0}(A)$ est un quotient de schémas en groupes affines et donc est un morphisme fidèlement plat de schémas affines. Ceci montre que le morphisme $\mathbb{R} S p e c, A \longrightarrow S p e c, H^{0}(A)$ possède une section après le changement de bases fidèlement plat $G_{0} \longrightarrow \operatorname{Spec} H^{0}(A)$. Ainsi, le morphisme de faisceaux

$$
\pi_{1}(F, *) \simeq \pi_{0}(\mathbb{R} \operatorname{Spec} A) \longrightarrow \operatorname{Spec}^{0}(A)
$$

est un épimorphisme.

Le théorème 3.2.4 possède aussi une réciproque.

Théorème 3.2.9. $\quad$ 1. Soit $F$ un type d'homotopie schématique pointé. Alors pour tout $i>0$ le faisceau $\pi_{i}(F, *)$ est représentable par un schéma en groupes affine qui est de plus unipotent pour $i>1$.

2. Soit $F$ une $\infty$-gerbe affine pointée. Alors les deux assertions suivantes sont vérifiées.

- Pour tout $i>1$, le faisceau $\pi_{i}(F, *)$ est représentable par un schéma en groupes affine et unipotent.

- Le faisceau $\pi_{1}(F, *)$ est un sous-faisceau d'un faisceau représentable par un schéma en groupes affine.

Preuve: Le point (1) ne sera pas démontré dans ce travail. Nous renvoyons à [32] pour une preuve. 
Démontrons la seconde assertion. Soit $A$ une $H_{\infty}$-algèbre de Hopf telle que $F \simeq B \mathbb{R}$ Spec $A$. Il existe un morphisme naturel de $H_{\infty}$-algèbres de Hopf

$$
H^{0}(A) \longrightarrow A
$$

qui induit un morphisme bien défini $\mathbb{R} \Omega_{*} F \longrightarrow S p e c H^{0}(A)$. Le lemme 3.2.8 implique alors que la fibre homotopique de ce morphisme est de la forme $\mathbb{R} S p e c B$, où $B$ est une $k$-algèbre co-simpliciale augmentée et cohomologiquement connexe. Le théorème 2.4 .5 et la suite longue des faisceaux d'homotopie permet alors de conlcure.

Nous terminerons ce paragraphe par la conjecture suivante.

Conjecture 3.2.10. Toute $\infty$-gerbe affine pointée est un type d'homotopie schématique pointé.

\subsection{Schématisation des types d'homotopie}

Pour tout $\mathbb{U}$-ensemble simplicial pointé $X$, nous noterons encore $X \in S \operatorname{Pr}(k)_{*}$ le préfaisceau simplicial pointé constant qu'il définit. Nous supposerons toujours que de tels ensembles simpliciaux sont pointés connexes. Il s'ensuit que les préfaisceaux simpliciaux $X$ seront donc pointés et connexes. Nous identifierons alors la catégorie des $\mathbb{U}$-ensembles simpliciaux pointés comme la sous-catégorie pleine de $\operatorname{SPr}(k)_{*}$ formée des préfaisceaux constants. Ceci nous permet d'appliquer les définitions de $\mathcal{O}$-équivalences et de $P$-équivalences aux morphismes d'ensembles simpliciaux pointés.

Définition 3.3.1. Soit $X$ un ensemble simplicial pointé et connexe de U. Une schématisation de $X$ sur $k$ est la donnée d'un type d'homotopie schématique pointé $(X \otimes k)^{s c h}$, et d'un morphisme dans la catégorie homotopique $\operatorname{Ho}\left(\operatorname{SPr}(k)_{*}\right)$, $u: X \longrightarrow(X \otimes k)^{s c h}$, qui soit universel pour les morphismes vers des types d'homotopie schématiques pointés.

Remarquer que si une schématisation existe alors elle est unique à isomorphisme unique près dans la catégorie homotopique. De même, tout morphisme $X \longrightarrow Y$ induira automatiquement un morphisme sur les schématisations (si elles existent) $(X \otimes k)^{s c h} \longrightarrow(Y \otimes k)^{s c h}$. Ces propriétés seront très pratiques par la suite car elles permettent de négliger les aspects fonctoriels des constructions.

Remarquer aussi qu'une schématisation est aussi une $P$-localisation (i.e. un morphisme universel vers les objets $P$-locaux $)$. Ainsi, pour démontrer que $(X \otimes$ $k)^{s c h}$ existe il suffit de trouver un type d'homotopie schématique $(X \otimes k)^{s c h}$ est une $P$-équivalence $X \longrightarrow(X \otimes k)^{s c h}$.

Le lemme suivant nous sera utile pour déterminer les $P$-équivalences entre ensembles simpliciaux.

Lemme 3.3.2. Un morphisme entre deux $\mathbb{U}$-ensembles simpliciaux pointés $f$ : $X \longrightarrow Y$ est une P-équivalence si et seulement si les deux conditions suivantes sont satisfaites. 
1. Le morphisme de groupes $f_{*}: \pi_{1}(X, *) \longrightarrow \pi_{1}(Y, *)$ induit un isomorphisme sur les complétés affines

$$
f_{*}: \mathcal{A}\left(\pi_{1}(X, *)\right) \simeq \mathcal{A}\left(\pi_{1}(Y, *)\right) .
$$

2. Pour tout système local de $k$-espaces vectoriels de dimension finie $L$ sur $Y$, et tout entier $i \geq 0$, le morphisme induit

$$
f^{*}: H^{i}(Y, L) \longrightarrow H^{i}\left(X, f^{*} L\right)
$$

est un isomorphisme.

Preuve: En appliquant directement la définition il est facile de voir qu'il suffit de démontrer que pour tout schéma en groupes affine et de type fini $G, V$ une de ses représentations linéaires de dimension finie, et tout $\mathbb{U}$-ensemble simplicial pointé et connexe $X$, il existe des isomorphismes naturels

$$
[X, K(G, V, n)]_{S P r(k)_{*}} \simeq[X, K(G(k), V(k), n)]_{\mathbb{U}-S E n s_{*}} .
$$

D'après le théorème 1.4 .3 on a

$$
[X, K(G, V, n)]_{S P r(k)_{*}} \simeq\left[\mathbb{R} \Omega_{*} X, G \times{ }_{\rho} K(V, n-1)\right]_{\operatorname{Pr}-\Delta^{o}-S \operatorname{Pr}(k)},
$$

où $G \times{ }_{\rho} K(V, n-1)$ est le préfaisceau en groupes simpliciaux produit semi-direct de $G$ par $K(V, n-1)$. L'adjonction de Quillen

$$
\begin{gathered}
C s t: \operatorname{Pr}-\Delta^{o}-S E n s \longrightarrow \operatorname{Pr}-\Delta^{o}-\operatorname{SPr}(k)_{*} \\
\Gamma: \operatorname{Pr}-\Delta^{o}-S \operatorname{Pr}(k)_{*} \longrightarrow \operatorname{Pr}-\Delta^{o}-S E n s
\end{gathered}
$$

donne

$\left[\mathbb{R} \Omega_{*} X, G \times{ }_{\rho} K(V, n-1)\right]_{P r-\Delta^{o}-S P r(k)} \simeq\left[\mathbb{R} \Omega_{*} X, \mathbb{R} \Gamma\left(G \times{ }_{\rho} K(V, n-1)\right)\right]_{P r-\Delta^{o}-S E n s}$. Cependant, le préfaisceau simplicial sous-jacent à $G \times{ }_{\rho} K(V, n-1)$ est $G \times K(V, n-$ 1). De plus, $V$ étant un faisceau en groupes sous-jacent à un faisceau quasi-cohérent il est acyclique sur $(A f f / k)_{f p q c}$. Ainsi, $G \times K(V, n-1)$ est un champ, et donc $G \times{ }_{\rho} K(V, n-1)$ est un objet fibrant dans $\operatorname{Pr}-\Delta^{o}-S \operatorname{Pr}(k)$. Ceci implique que

$$
\mathbb{R} \Gamma\left(G \times{ }_{\rho} K(V, n-1)\right) \simeq G(k) \times{ }_{\rho} K(V(k), n-1) .
$$

On en déduit donc

$$
\begin{gathered}
{[X, K(G, V, n)]_{S P r}(k)_{*} \simeq\left[\mathbb{R} \Omega_{*} X, G(k) \times_{\rho} K(V(k), n-1)\right]_{P r-\Delta^{o}-S E n s}} \\
\simeq[X, K(G(k), V(k), n)]_{\mathbb{U}-S E n s_{*}},
\end{gathered}
$$

ce qu'il fallait démontrer.

Corollaire 3.3.3. Soit $X$ un $\mathbb{U}$-ensemble simplicial pointé et connexe, $G$ un schéma en groupe affine et $V$ une représentation linéaire de $G$. Alors, il existe un isomorphisme naturel

$$
\mathbb{R H o m}_{*}(X, K(G, V, n)) \simeq \mathbb{R} \underline{\operatorname{Hom}}_{*}(X, K(G(k), V(k), n)) .
$$


Preuve: C'est en réalité un corollaire de la preuve du lemme 3.3.2.

Le théorème principal de ce paragraphe est le suivant.

Théorème 3.3.4. Tout $\mathbb{U}$-ensemble simplicial pointé et connexe $(X, x)$ possède une schématisation $(X \otimes k)^{\text {sch }}$.

Preuve: Nous allons donner une construction explicite du type d'homotopie schématique pointé $(X \otimes k)^{s c h}$, et nous montrerons que le morphisme $X \longrightarrow(X \otimes$ $k)^{s c h}$ est une $P$-équivalence. Comme les types d'homotopie schématiques pointés sont des objets $P$-locaux, ceci impliquera que ce morphisme est une schématisation.

Considérons le foncteur classifiant $B: \mathbb{U}-S G p \longrightarrow \mathbb{U}-S E n s_{*}$, qui à un $\mathbb{U}$-groupe simplicial $G$ associe son ensemble simplicial pointé classifiant $B G$. On sait que ce foncteur induit un foncteur au niveau des catégories homotopiques $B: H o(\mathbb{U}-S G p) \longrightarrow H o\left(\mathbb{U}-S E n s_{*}\right)$, qui est pleinement fidèle et dont l'image essentielle est formée des ensembles simpliciaux connexes (voir [57, Prop. 1.5] pour une preuve de ce fait bien connue). Il existe donc un $\mathbb{U}$-groupe simplicial $G$ tel que $B G$ soit équivalent à $X$. De plus, quitte à prendre une résolution libre de $G$ on pourra supposer que chaque groupe $G_{n}$ est un groupe libre de $\mathbb{U}$.

Considérons alors $\mathcal{A}(G)$ le schéma en groupes affine simplicial déduit de $G$ en appliquant la construction de complétion affine (voir Def. 1.5.3). On définit le champ $(X \otimes k)^{s c h}$ comme étant $B \mathbb{R} \operatorname{Spec} \mathcal{O}\left(h_{\mathcal{A}(G)}\right) \in \operatorname{Ho}\left(\operatorname{SPr}(k)_{*}\right)$, dont la construction est expliquée dans le paragraphe 2.3. Nous savons donc déjà que $(X \otimes k)^{s c h}$ est une $\infty$-gerbe affine pointée, et que le morphisme naturel $B \mathcal{A}(G) \longrightarrow$ $(X \otimes k)^{s c h}$ est de plus universel pour les morphismes vers des $\infty$-gerbes affines pointées (voir Lem. 3.1.5). De plus, le corollaire 3.2.7 montre que $(X \otimes k)^{s c h}$ est un type d'homotopie schématique pointé. Il nous reste à montrer que le morphisme naturel $X \longrightarrow(X \otimes k)^{\text {sch }}$ est une $P$-équivalence.

Nous avons défini $(X \otimes k)^{s c h}$ comme étant $B \mathbb{R} \operatorname{Spec} \mathcal{O}(\mathcal{A}(G))$. On dispose donc des morphismes naturels

$$
X \simeq B G \stackrel{a}{\longrightarrow} B \mathcal{A}(G) \stackrel{b}{\longrightarrow} B \mathbb{R} \operatorname{Spec} \mathcal{O}\left(h_{\mathcal{A}(G)}\right)=:(X \otimes k)^{s c h},
$$

et il nous suffit donc de montrer que $a$ et $b$ sont des $P$-équivalences. Pour le morphisme $b$ c'est immédiat d'après sa propriété universelle (voir Lem. 3.1.5), et le fait que $K(K, V, N)$ est une $\infty$-gerbe affine pointée dès que $K$ est un schéma en groupes affine et $V$ une de ses représentations linéaire de dimension finie. Pour terminer montrons que le morphisme $a: B G \longrightarrow B \mathcal{A}(G)$ est une $P$-équivalence.

Tout d'abord en utilisant les équivalences

$$
B G \simeq \text { Hocolim }_{[n] \in \Delta^{\circ}} B G_{n} \quad B \mathcal{A}(G) \simeq \text { Hocolim }_{[n] \in \Delta^{\circ}} B \mathcal{A}\left(G_{n}\right)
$$

on voit qu'il suffit de montrer que pour tout $n$ le morphisme $B G_{n} \longrightarrow B \mathcal{A}\left(G_{n}\right)$ est une $P$-équivalence. 
Soit $H$ un schéma en groupes affine et de type fini sur $k, V \in \operatorname{Rep}_{k}(H)$ une représentation linéaire de dimension finie et $F=K(H, V, n)$. D'après le corollaire 3.3.3 il faut montrer que

$$
\mathbb{R} \underline{\operatorname{Hom}}_{*}\left(B G_{n}, F(k)\right) \simeq \mathbb{R} \underline{\operatorname{Hom}}_{*}\left(B \mathcal{A}\left(G_{n}\right), F\right) .
$$

Or, il existe un diagramme commutatif

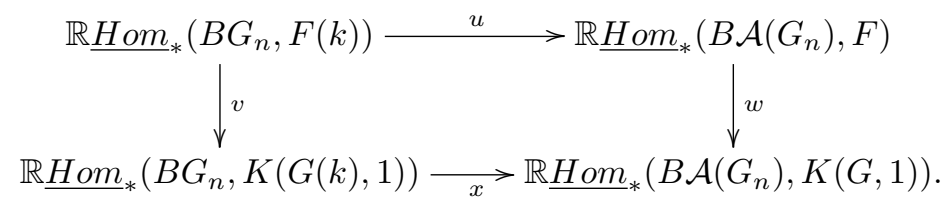

De plus, la propriété universelle du morphisme $G_{n} \longrightarrow \mathcal{A}\left(G_{n}\right)$ implique clairement que $x$ est un isomorphisme. Il nous suffit donc de montrer que $u$ induit des isomorphismes sur toutes les fibres homotopiques des morphismes $v$ et $w$. Or, si $\rho: G_{n} \longrightarrow G(k)$ est un point dans $\pi_{0}\left(\mathbb{R}_{\text {Hom }_{*}}\left(B G_{n}, K(G(k), 1)\right)\right)$, et si on note $v^{-1}(\rho)$ et $w^{-1}(\rho)$ ces fibres homotopiques, on a

$$
\pi_{i}\left(v^{-1}(\rho)\right) \simeq H^{n-i}\left(B G_{n}, V(k)\right) \quad \pi_{i}\left(w^{-1}(\rho)\right) \simeq H^{n-i}\left(B \mathcal{A}\left(G_{n}\right), V\right) .
$$

On se ramène donc à montrer le lemme suivant.

Lemme 3.3.5. Pour tout entier $n$, et toute représentation linéaire de dimension finie $V$ de $\mathcal{A}\left(G_{n}\right)$, on a

$$
H^{i}\left(B \mathcal{A}(G)_{n}, V\right) \simeq H^{i}\left(B G_{n}, V\right)
$$

pour tout entier $i \geq 0$.

Preuve: Commençons par montrer que $H^{i}\left(B \mathcal{A}(G)_{n}, V\right) \simeq H^{i}\left(B G_{n}, V\right) \simeq$ 0 pour $i>1$. Pour ce qui est de l'annulation de $H^{i}\left(B G_{n}, V\right)$ cela provient immédiatement du fait que $G_{n}$ soit libre et du corollaire 3.3.3. Ainsi, d'après le lemme 1.5.1 il nous suffit de montrer que pour toute représentation linéaire $W$ (éventuellement de dimensions infinie) on a $H^{2}\left(B \mathcal{A}\left(G_{n}\right), W\right)=0$. Comme une telle représentation est limite inductive de ses sous-représentations de dimension finie on peut à l'aide du corollaire 1.5.2 se restreindre au cas où $W$ est de dimension finie.

Il est facile de voir que pour montrer que $H^{2}\left(B \mathcal{A}\left(G_{n}\right), W\right)=0$ il suffit de montrer que toute extension de faisceaux en groupes

$$
0 \longrightarrow W \longrightarrow E \stackrel{p}{\longrightarrow} \mathcal{A}\left(G_{n}\right) \longrightarrow 1
$$

possède une section. Cependant, comme $E$ est un $W$-torseur sur $\mathcal{A}\left(G_{n}\right)$ qui est un schéma affine, le morphisme $p$ possède une section dans la catégorie des schémas $s: \mathcal{A}\left(G_{n}\right) \longrightarrow E$. Remarquons aussi que $E$ est représentable par un schéma en groupes affine. Ainsi, si le groupe $G_{n}$ est un groupe libre sur un U-ensemble $I$, le morphisme de préfaisceaux

$$
I \longrightarrow G_{n} \longrightarrow \mathcal{A}\left(G_{n}\right) \stackrel{s}{\longrightarrow} E
$$


se relève en un morphisme de groupes $G_{n} \longrightarrow E$. Ce morphisme induit alors un morphisme de schémas en groupes $\mathcal{A}\left(G_{n}\right) \longrightarrow E$, qui est la section cherchée.

Il nous reste donc à vérifier que $H^{i}\left(B \mathcal{A}\left(G_{n}\right), V\right) \simeq H^{i}\left(B G_{n}, V\right)$ pour $i<2$. Mais ceci est vrai pour tout groupe $G_{n}$, libre ou pas, et se déduit aisément de la propriété universelle des complétions affines (voir par exemple [36, §4]).

Ce lemme permet de conlure que le morphisme $a$ est une équivalence, et termine donc la preuve du théorème.

Notons $H o(T H S / k)$ la sous-catégorie pleine de $H o\left(S \operatorname{Pr}(k)_{*}\right)$ formée des types d'homotopie schématiques pointés. Considérons le foncteur dérivé des sections globales

$$
\mathbb{R} \Gamma: H o(T H S / k) \subset H o\left(S P r_{*}(k)\right) \longrightarrow H o\left(\mathbb{V}-S E n s_{*}\right) .
$$

En se restreignant à la composante connexe qui contient le point distingué on en déduit un foncteur

$$
\mathbb{R} \Gamma: H o(T H S / k) \longrightarrow H o\left(\mathbb{V}-S E n s_{0}\right),
$$

où $H o\left(\mathbb{V}-S E n s_{0}\right)$ est la catégorie homotopique des $\mathbb{V}$-ensembles simpliciaux pointés et connexes. En utilisant le théorème 1.4.3 et le corollaire 2.2.3, on voit que le foncteur

se factorise en un foncteur

$$
\mathbb{R} \Gamma: H o(T H S / k) \longrightarrow H o\left(\mathbb{V}-S E n s_{0}\right)
$$

$$
\mathbb{R} \Gamma: H o(T H S / k) \longrightarrow H o\left(\mathbb{U}-S E n s_{0}\right) .
$$

Corollaire 3.3.6. Le foncteurs dérivé du foncteur des sections globales

$$
\mathbb{R} \Gamma: H o(T H S / k) \longrightarrow H o\left(\mathbb{U}-S E n s_{0}\right)
$$

possède un adjoint à gauche

$$
(-\otimes k)^{s c h}: H o\left(\mathbb{U}-S E n s_{0}\right) \longrightarrow H o(T H S / k) .
$$

Preuve: C'est une autre façon d'énoncer le théorème 3.3.4.

Corollaire 3.3.7. Pour tout ensemble simplicial pointé et connexe $(X, x)$ appartenant à $\mathbb{U}$, il existe un isomorphisme naturel

$$
\pi_{1}\left((X \otimes k)^{s c h}, x\right) \simeq \mathcal{A}\left(\pi_{1}(X, x)\right) .
$$

Preuve: On sait d'après le théorème 3.2 .9 que $\pi_{1}(X \otimes, k, x)$ est un schéma en groupes affine. De plus, comme $X \longrightarrow(X \otimes k)^{s c h}$ est une $P$-équivalence, 3.2.4 implique que'on a pour tout schéma en groupes affine $G$

$$
\operatorname{Hom}\left(\pi_{1}(X, x), G\right) \simeq \operatorname{Hom}\left(\pi_{1}\left((X \otimes k)^{s c h}, x\right), G\right) .
$$

On conclut alors à l'aide de la propriété universelle de $\mathcal{A}\left(\pi_{1}(X, x)\right)$. 
Corollaire 3.3.8. Soit $X$ un $\mathbb{U}$-ensemble simplicial pointé et connexe. Si le champ $(X \otimes k)^{\text {sch }}$ est simplement connexe (i.e. $\left.\pi_{1}(X \otimes k) \simeq 0\right)$, alors il existe un isomorphisme naturel

$$
(X \otimes k)^{s c h} \longrightarrow(X \otimes k)^{u n i} .
$$

Preuve: Comme $(X \otimes k)^{\text {sch }}$ est simplement connexe, c'est un champ affine d'après 2.4.1 et 3.3.4. De plus, $X \longrightarrow(X \otimes k)^{s c h}$ étant une $P$-équivalence, c'est aussi une $\mathcal{O}$-équivalence. C'est donc une affination.

Remarquer qu'en général il existe toujours un morphisme naturel

$$
(X \otimes k)^{s c h} \longrightarrow(X \otimes k)^{u n i} .
$$

Corollaire 3.3.9. Soit $X$ un $\mathbb{U}$-ensemble simplicial pointé, simplement connexe et de type fini. Alors on a

$$
\pi_{i}\left((X \otimes k)^{s c h}, x\right) \simeq \begin{cases}\pi_{i}(X, x) \otimes_{\mathbb{Z}} \mathbb{G}_{a} & \text { si car } k=0 \\ \pi_{i}(X, x) \otimes_{\mathbb{Z}} \mathbb{Z}_{p} & \text { si car } k=p .\end{cases}
$$

Preuve: Ceci se déduit des corollaires 3.3 .8 et 2.5.3.

\subsection{Exemples et contre-exemples}

Dans ce paragraphe nous donnons une liste d'exemples et de contre-exemples du comportement du foncteur de schématisation. Nous ne donnerons pas de preuves étant donné que les arguments se déduisent ou bien de la propriété universelle, ou bien des résultats des deux paragraphes précédents.

- Reprenons l'exemple cité dans le paragraphe 2.6, d'un type d'homotopie $X=$ $K\left(\mathbb{Z} / m, \mathbb{Z}^{r}, n\right)$. Il n'est pas difficile de voir dans ce cas que

$$
(X \otimes \mathbb{Q})^{s c h} \simeq K\left(\mathbb{Z} / m, \mathbb{G}_{a}^{r}, n\right),
$$

et où l'action de $\mathbb{Z} / m$ sur $\mathbb{G}_{a}^{r}$ est la $\mathbb{Q}$-linéarisée de l'action sur $\mathbb{Z}^{r}$.

- Il est bien connu qu'il est généralement impossible de donner une formule pour les faisceaux d'homotopie du champ $(X \otimes k)^{u n i}$ lorsque $X$ n'est pas nilpotent. Il est donc aussi impossible de donner une formule générale pour les faisceaux d'homotopie du champ $(X \otimes k)^{s c h}$. Cela peut en réalité déjà se remarquer sur le cas 1-tronqué.

Si $\Gamma$ est un $\mathbb{U}$-groupe, alors on sait que $\pi_{1}\left((K(\Gamma, 1) \otimes k)^{s c h}, *\right)$ est le complété affine de $\Gamma$ sur $k$. Par contre, il n'est pas vrai en général que $(K(\Gamma, 1) \otimes k)^{s c h} \simeq K(\mathcal{A}(\Gamma), 1)$. La raison en est que le groupe $\Gamma$ peut très bien ne pas avoir la même cohomologie que $\mathcal{A}(\Gamma)$. Le phénomène qui se produit ici est de nature tout à fait équivalente à ce qu'il se produit lors des complétions pro-finies (voir [1]). On peut donc introduire un analogue algébrique de la notion de groupe bon.

Définition 3.4.1. Le groupe $\Gamma$ est bon sur $k$ si le morphisme naturel

$$
(K(\Gamma, 1) \otimes k)^{s c h} \longrightarrow K(\mathcal{A}(\Gamma), 1)
$$


est un isomorphisme dans $\operatorname{Ho}\left(\operatorname{SPr}(k)_{*}\right)$.

Il est par exemple facile de voir que les groupes libres sont bons sur n'importe quel corps $k$ (ceci provient du lemme 3.3.5). De même, les groupes abéliens de types finis, les groupes fondamentaux de surfaces de Riemann et les extensions succésives de groupes libres de types finis sont tous des exemples de groupes bons (voir [32]). Par contre un groupe abélien qui n'est pas de type fini n'est généralement pas bon, et ce même si $k=\mathbb{Q}$.

On en déduit par exemple que

$$
(K(\mathbb{Z}, 1) \otimes k)^{s c h} \simeq K(\mathcal{A}(\mathbb{Z}), 1) .
$$

On peut de plus calculer explicitement le schéma groupe affine $\mathcal{A}(\mathbb{Z})$. Pour cela, considérons $\bar{k}^{*}$, le groupe discret multiplicatif de la clôture algébrique du corps $k$. Il est muni d'une action naturelle du groupe de galois $\operatorname{Gal}(\bar{k} / k)$. Formons alors le schéma en groupes de type multiplicatif $D\left(\bar{k}^{*}\right)$, tel que son faisceau des caractères soit isomorphe à $\bar{k}^{*}$, muni de son action galoisienne. On peut alors montrer qu'il existe un isomorphisme (voir par exemple [44, App. A])

$$
\mathcal{A}(\mathbb{Z}) \simeq D\left(\bar{k}^{*}\right) \times \mathcal{U}(\mathbb{Z})
$$

et donc que la schématisation de $K(\mathbb{Z}, 1)$ est donnée par

$$
(K(\mathbb{Z}, 1) \otimes k)^{s c h} \simeq K\left(D\left(\bar{k}^{*}\right), 1\right) \times K(\mathcal{U}(\mathbb{Z}), 1) .
$$

Ceci implique que le morphisme naturel

$$
(K(\mathbb{Z}, 1) \otimes k)^{s c h} \longrightarrow(K(\mathbb{Z}, 1) \otimes k)^{u n i}
$$

possède une fibre homotopique équivalente à $K\left(D\left(\bar{k}^{*}\right), 1\right)$, qui est un champ énorme. On voit ainsi, que même dans les cas les plus simples, lorsque $X$ n'est pas simplement connexe le champ $(X \otimes k)^{s c h}$ est généralement beaucoup plus gros que le champ $(X \otimes k)^{u n i}$. Déjà au niveau du groupe fondamental, le morphisme $\pi_{1}\left((X \otimes k)^{s c h}, *\right) \longrightarrow \pi_{1}\left((X \otimes k)^{u n i}, *\right)$ possède un très gros noyau. Il s'ensuit à fortiori que la fibre homotopique de $(X \otimes k)^{s c h} \longrightarrow(X \otimes k)^{u n i}$ est aussi énorme.

Une autre conséquence remarquable du fait que $\pi_{1}\left((X \otimes k)^{s c h}, *\right) \simeq$ $\mathcal{A}\left(\pi_{1}(X, *)\right)$ est que la formation de la schématisation $(X \otimes k)^{s c h}$ ne commute pas avec les changements de bases en général. Par exemple, il n'est pas vrai que $D\left(\overline{\mathbb{Q}}^{*}\right) \times_{\text {Spec }} \overline{\mathbb{Q}} \operatorname{Spec} \mathbb{C} \simeq D\left(\mathbb{C}^{*}\right)$. Ceci est une différence importante avec ce qui se passe pour les affinations (voir Cor. 2.3.5).

- Lorsque $k$ est de caractéristique nulle, la schématisation $(X \otimes k)^{s c h}$ possède une décomposition naturelle en une partie réductive et une partie unipotente, analogue à la décomposition d'un schéma en groupes affine (voir [25, 4]).

Considérons la projection naturelle

$$
(X \otimes k)^{s c h} \longrightarrow K\left(\pi_{1}\left((X \otimes k)^{s c h}, *\right), 1\right),
$$


ainsi que le quotient maximal réductif

$$
\pi_{1}\left((X \otimes k)^{s c h}, *\right) \longrightarrow \pi_{1}\left((X \otimes k)^{s c h}, *\right)^{r e d} .
$$

Par définition, $\pi_{1}\left((X \otimes k)^{s c h}, *\right)^{r e d}$ est la limite projective des quotients réductifs et de type fini de $\pi_{1}\left((X \otimes k)^{s c h}, *\right)$, et se trouve être isomorphe à l'enveloppe réductive de $\pi_{1}(X, *)$. Le noyau de la projection $\pi_{1}((X \otimes$ $\left.k)^{s c h}, *\right) \longrightarrow \pi_{1}\left((X \otimes k)^{s c h}, *\right)^{\text {red }}$ est un schéma en groupe affine et unipotent. Ainsi, la fibre homotopique du morphisme

$$
(X \otimes k)^{s c h} \longrightarrow K\left(\pi_{1}\left((X \otimes k)^{s c h}, *\right)^{r e d}, 1\right)
$$

est un champ connexe dont tous les faisceaux d'homotopie sont représentables par des schémas en groupes unipotents. D'après le théorème 2.4.1, c'est donc un champ affine. Il existe donc une $k$-algèbre co-simpliciale $A$ dans $\mathbb{U}$, une action de $\pi_{1}\left((X \otimes k)^{s c h}, *\right)^{\text {red }}$ sur le champ affine $(X \otimes k)^{o}:=\mathbb{R}$ Spec $A$, tel que $(X \otimes k)^{s c h}$ soit le quotient homotopique de $(X \otimes k)^{o}$ par l'action de $\pi_{1}\left((X \otimes k)^{s c h}, *\right)$. De plus, le schéma en groupes $\pi_{1}\left((X \otimes k)^{s c h}, *\right)^{r e d}$ étant réductif et $k$ de caractéristique nulle, sa cohomologie à valeurs dans des représentations linéaires est nulle, et on a donc pour toute représentation linéaire $\rho: \pi_{1}\left((X \otimes k)^{s c h}, *\right) \longrightarrow G l(V)$

$$
H^{i}\left((X \otimes k)^{s c h}, V\right) \simeq H^{i}\left((X \otimes k)^{o}, V\right)^{\pi_{1}\left((X \otimes k)^{s c h}, *\right)^{r e d}} .
$$

La $k$-algèbre $A$, munie de l'action de $\pi_{1}\left((X \otimes k)^{s c h}, *\right)^{\text {red }}$ sur $\mathbb{R} S p e c A$, répond ainsi à une question posée par A. Beilinson, T. Pantev et L. Katzarkov, et qui avait été abordée par des méthodes différentes lorsque $X$ était l'espace topologique sous-jacent à une variété algébrique lisse sur $\mathbb{C}$. Cette construction est aussi liée à un modèle explicite pour le champ $(X \otimes k)^{s c h}$ décrit à l'aide d'une notion de champs affines équivariants (voir [31]).

- Nous terminerons ces exemples par un exemple qui montre que bien que le champ $(X \otimes k)^{\text {sch }}$ contienne beaucoup d'information homotopique il ne suffit pas à reconstruire $X$. Pour cela nous allons construire un morphisme $f: X \longrightarrow Y$ entre deux U-ensembles simpliciaux pointés, qui induit des équivalences $(X \otimes k)^{s c h} \longrightarrow(Y \otimes k)^{s c h}$ pour tout $\mathbb{U}$-corps $k$, mais qui n'est pas une équivalence faible. Cet exemple m'a été communiqué par C. Simpson.

D'après [8] il existe un groupe $\Gamma$ qui est de présentation finie, infini et simple. Posons alors $X=K(\Gamma, 1)$ et prenons pour $Y$ la construction + de Quillen $Y:=X^{+}$, muni du morphisme naturel $f: X \longrightarrow Y$. L'ensemble simplicial $Y$ est donc simplement connexe et le morphisme $f$ est une équivalence de cohomologie (à coefficients dans $\mathbb{Z}$ et donc dans tout corps $k$ ). Remarquer dès à présent que $f$ n'est pas une équivalence. D'après le corollaire 3.3.7, on a pour tout corps $k$

$$
\pi_{1}\left((X \otimes k)^{s c h}\right) \simeq \mathcal{A}\left(\pi_{1}(X, x)\right) \simeq \mathcal{A}(\Gamma) .
$$

Or, le groupe $\Gamma$ étant simple, infini et de présentation fini il ne possède aucune représentation linéaire sur $k$ qui soit non triviale (car tout sous-groupe de présentation finie de $G l_{n}(k)$ possède des sous-groupes d'indices finis non 
triviaux $)$. Le champ $(X \otimes k)^{\text {sch }}$ est donc simplement connexe, et on a donc $(X \otimes k)^{s c h} \simeq(X \otimes k)^{\text {uni }}$ (voir Cor. 3.3.8). Or, comme $f$ est une équivalence de cohomologie le morphisme induit

$$
(X \otimes k)^{s c h} \simeq(X \otimes k)^{u n i} \longrightarrow(Y \otimes k)^{s c h} \simeq(Y \otimes k)^{u n i}
$$

est une équivalence.

\subsection{Types d'homotopie des variétés algébriques}

Le but de ce paragraphe est d'ésquisser quelques applications de la notion de types d'homotopie schématiques dans le cadre de la géométrie algébrique. Nous ne donnerons pas de détails ni de preuves qui apparaitront dans des travaux ultérieurs. Notons au passage que les constructions ci-dessous peuvent être facilement obtenues à l'aide des techniques utilisées dans [31, 40], basées sur la notion de champs affine équivariants. Elles peuvent être aussi obtenues, tout au moins conjecturalement, en appliquant le formalisme des catégories de Segal Tannakiennes (voir [58] et [59] pour plus de détails).

On rappelle que $H o(T H S / k)$ est le sous-catégorie pleine de $H o\left(S P r_{*}(k)\right)$ formée des types d'homotopie schématiques pointés sur le corps $k$.

3.5.1. Type d'homotopie schématiques et théorie de Hodge. Soit $X$ une variété lisse, connexe et projective sur $\mathbb{C}$, et $x \in X$. On peut associer à $X$ plusieurs types d'homotopie schématiques sur $\mathbb{C}$, chacun d'eux associés aux théories cohomologiques de Betti, de de Rham et de Dolbeault. La théorie de Hodge nonabélienne de [23] et la correspondence de Riemann-Hilbert permettent alors de construire des isomorphismes de comparaison entre ces types d'homotopie, et de construire une certaine décomposition de Hodge qui englobe les décompositions de Hodge usuelles sur la cohomologie, l'homotopie rationnelle et le groupe fondamental.

- Notons $X^{\text {top }}$ l'espace topologique des points complexes de $X$ muni de la topologie analytique. On dispose alors du type d'homotopie schématique pointé $\left(X^{t o p} \otimes \mathbb{C}\right)^{s c h}$, schématisation du type d'homotopie de $X^{t o p}$. La propriété universelle de la schématisation nous dit que la cohomologie à coefficients locaux de $\left(X^{t o p} \otimes \mathbb{C}\right)^{\text {sch }}$ calcule la cohomologie de Betti de $X$ (i.e. la cohomologie de l'espace $X^{\text {top }}$ à valeurs dans des systèmes locaux de $\mathbb{C}$ vectoriels de dimension finis).

- On associe à $X$ un préfaisceau $X_{D R}$ défini par la formule

$$
X_{D R}(A):=X\left(A_{\text {red }}\right),
$$

pour toute $\mathbb{C}$-algèbre $A$, et où $A_{\text {red }}$ est la $\mathbb{C}$-algèbre réduite (voir [51]). On considère $X_{D R}$ comme un objet de $H o\left(\operatorname{SPr}_{*}(\mathbb{C})\right)$, et donc comme un champ pointé sur $\mathbb{C}$. On peut alors montrer (par exemple en utilisant les techniques 
utilisées dans [31]) que le foncteur

$$
\begin{array}{clc}
H o(T H S / \mathbb{C}) & \longrightarrow & \text { Ens } \\
F & \mapsto & \left.\left[X_{D R}, F\right]_{H o(S P r}(\mathbb{C})\right)
\end{array}
$$

est co-représentable par un type d'homotopie schématique pointé, que l'on notera $(X, x)^{D R}$, et qui est appelé le type d'homotopie schématique de de Rham de $(X, x)$.

Par définition il existe une équivalence tensorielle entre la catégorie tensorielle des représentations linéaires de dimension finies de $\pi_{1}\left((X, x)^{D R}, *\right)$ et celle des fibrés vectoriels algébriques munis de connexions intégrables sur $X$. En d'autres termes, le schéma en groupes $\pi_{1}\left((X, x)^{D R}, *\right)$ est le dual de Tannaka de la catégorie des fibrés plats sur $X$. De plus, pour une telle représentation $V$, la cohomologie du champ $(X, x)^{D R}$ à valeurs dans $V$ s'identifie naturellement avec la cohomologie de de Rham algébrique de $X$ à valeurs dans la connexion correspondante.

$$
H^{*}\left((X, x)^{D R}, V\right) \simeq H_{D R}^{*}(X, V) .
$$

- La correspondence de Riemann-Hilbert interprétée de façon adéquate permet de construire un isomorphisme naturel de champs pointés

$$
\rho_{R H}:\left(X^{t o p} \otimes \mathbb{C}\right)^{s c h} \simeq(X, x)^{D R} .
$$

En d'autres termes, il existe un morphisme naturel de champs pointés

$$
(X, x) \longrightarrow(X, x)^{D R},
$$

que l'on pourrait appelé application des périodes, et qui induit par propriété universelle de la schématisation l'isomorphisme $\rho_{R H}$. Cette équivalence est une généralisation de l'isomorphisme de comparaison bien connu entre cohomologie de de Rham et cohomologie de Betti au cas des types d'homotopie.

- À $X$, on peut aussi associer le champ $X_{D o l}$, qui par définition est le champ classifiant du $X$-schéma en groupes formel $\hat{T X}$, complété formel du fibré tangent (vu comme schéma en groupes sur $X$, voir [51]). On considére $X_{D o l}$ comme un champ pointé (en $x \in X(\mathbb{C})$ ), et donc comme un objet de la catégorie $\mathrm{Ho}\left(\mathrm{SPr}_{*}(\mathbb{C})\right)$. Comme précédemment, on définit un foncteur

$$
\begin{array}{clc}
H o(T H S / \mathbb{C}) & \longrightarrow & \text { Ens } \\
F & \mapsto & \left.\left[X_{D o l}, F\right]_{H o(S P r}(\mathbb{C})\right)
\end{array}
$$

et l'on considère un sous-foncteur défini comme suit. À un morphism de champs pointés $X_{D o l} \longrightarrow F$, où $F$ est un type d'homotopie schématique pointé, on peut associer le morphism induit par composition avec la projection $F \longrightarrow \tau_{\leq 1} F \simeq K(H, 1)$, où $H=\pi_{1}(F, *)$. Ce morphisme correspond à une classe d'isomorphisme de $H$-torseur sur le champ $X_{D o l}$, où de manière équivalente à un $H$-fibré principal de Higgs sur $X$ (comme défini dans [50]), que nous noterons $P$. Pour tout représentation linéaire de dimension finie $V$ de $H$, ce $H$-fibré principal de Higgs donne lieu à un fibré de Higgs $P \times{ }^{H} V$. Nous dirons alors que le morphisme $X_{\text {Dol }} \longrightarrow F$ est semi-stable de 
degré 0 si pour tout représentation linéaire de dimension finie $V$ le fibré de Higgs $P \times{ }^{H} V$ est semi-stable et de degré 0 au sens de [50]. Si l'on note $\operatorname{par}\left[X_{D o l}, F\right]_{H o\left(S P r_{*}(\mathbb{C})\right)}^{s s, 0}$ le sous-ensemble de $\left[X_{D o l}, F\right]_{H o\left(S P r_{*}(\mathbb{C})\right)}$ formé des morphismes semi-stables de degré 0 on obtient ainsi un foncteur

$$
\begin{array}{ccc}
H o(T H S / \mathbb{C}) & \longrightarrow & \text { Set } \\
F & \mapsto & {\left[X_{D o l}, F\right]_{H o\left(S P r_{*}(\mathbb{C})\right)^{s s}, 0}}
\end{array}
$$

On montre alors (par exemple à l'aide des techniques de constructions de [31]) que ce foncteur est co-représentable par un type d'homotopie schématique pointé $(X, x)^{D o l}$, et appelé le type d'homotopie de Dolbeault de $X$.

- La correspondence fondamentale de la théorie de Hodge non-abélienne, telle qu'énoncée dans [50, Lem. 2.2], permet de construire un isomorphisme naturel de champs pointés

$$
\rho_{\text {Hod }}:(X, x)^{D o l} \simeq(X, x)^{D R} .
$$

Il existe donc un diagramme d'isomorphismes naturels de champs pointés

$$
(X, x)^{D o l} \stackrel{\rho_{H o d}}{\longrightarrow}(X, x)^{D R} \stackrel{\rho_{R H}}{\prec}\left(X^{t o p} \otimes \mathbb{C}\right)^{s c h} .
$$

Le groupe (discret) $\mathbb{C}^{\times}$opère par homothéties sur le $X$-schéma en groupes $T X$, et donc par fonctorialité sur le champ $X_{D o l}$. Ceci induit, à travers les isomorphismes ci-dessus une action de $\mathbb{C}^{\times}$sur le champ $\left(X^{\text {top }} \otimes \mathbb{C}\right)^{\text {sch }}$. Cette action n'est pas uniquement une action dans la catégorie homotopique des champs pointés, mais existe de facçon naturelle en tant qu'objet de la catégorie homotopique des préfaisceaux simpliciaux pointés $\mathbb{C}^{\times}$-équivariants (voir [31] pour plus de détails). Cette action de $\mathbb{C}^{\times}$sur $\left(X^{\text {top }} \otimes \mathbb{C}\right)^{\text {sch }}$ est par définition la décomposition de Hodge de $\left(X^{t o p} \otimes \mathbb{C}\right)^{s c h}$. Cette terminologie est justifiée par le théorème principal de [31] qui affirme que l'action de $\mathbb{C}^{\times}$ permet de retrouver les structures de Hodge sur la cohomologie, l'homotopie rationnelle et le groupe fondamental de $X$.

- Le champ $\left(X^{t o p} \otimes \mathbb{C}\right)^{\text {sch }}$, muni de sa décomposition de Hodge me semble un invariant digne d'intérêt. Par exemple, dans [31] on montre comment on peut obtenir des exemples de types d'homotopie qui ne sont pas réalisables par des variétés complexes lisses et projectives, et dont l'obstruction à la réalisabilité se trouve dans des invariants d'homotopie supérieure (essentiellement l'action du groupe fondamental sur les groupes d'homotopie). Une question qui reste à étudier est le problème de type Torelli, c'est à dire la reconstruction de certain type de variétés algébriques à partir du champ $\left(X^{\text {top }} \otimes \mathbb{C}\right)^{\text {sch }}$ muni de sa décomposition de Hodge.

- Pour finir avec la théorie de Hodge, signalons qu'il existe aussi une variante de la construction précédente, qui à $(X, x)$ associe un type d'homotopie de Hodge $X^{\mathbb{H}}$. Il s'agit d'un type d'homotopie schématique pointé sur $\mathbb{C}$, muni d'un morphisme naturel

$$
X^{\mathbb{H}} \longrightarrow K\left(\mathbb{G}_{m}, 1\right) .
$$


La fibre homotopique de ce morphisme est notée $\bar{X}^{\mathbb{H}}$, et appelé le type d'homotopie de Hodge géométrique de $X$. Les systèmes locaux sur $\bar{X}^{\mathbb{H}}$ correspondent aux systèmes locaux qui peuvent être munis d'une structure de variation de structures de Hodge complexes polarizabes sur $X$. De plus la cohomologie de $\bar{X}^{\mathbb{H}}$ à coefficients dans un tel système local s'identifie à la cohomologie de $X$ à valeurs dans la variation de structure de Hodge correspondante. En particulier, pour un tel sytème local, l'action de $\mathbb{G}_{m}$ induite sur $H^{n}\left(\bar{X}^{\mathbb{H}}, L\right)$ correspond à la décomposition de Hodge

$$
H^{n}(X, L) \simeq \bigoplus_{p+q=n} H^{p}\left(X, L \otimes \Omega^{q}\right)
$$

en opérant par poids $q$ sur la composante $H^{p}\left(X, L \otimes \Omega^{q}\right)$. Il existe de plus, un morphisme naturel de types d'homotopie schématiques

$$
(X \otimes \mathbb{C})^{s c h} \longrightarrow \bar{X}^{\mathbb{H}},
$$

qui est $\mathbb{C}^{\times}$-équivariant. D'une certain façon, ce morphisme est le quotient maximal de $(X \otimes \mathbb{C})^{\text {sch }}$ sur lequel le groupe $\mathbb{C}^{\times}$opère algébriquement.

Il existe aussi des versions plus fines de $X^{\mathbb{H}}$ correspondants aux variations de structures de Hodge réelles, voir rationnelles. Les types d'homotopie schématiques sont alors définis sur $\mathbb{R}$ ou sur $\mathbb{Q}$. Le groupe $\mathbb{G}_{m}$ est alors remplacé par le groupe de Tannaka de la catégorie des structures de Hodge réelles ou rationnelles. Les systèmes locaux sur $X^{\mathbb{H}}$ correspondent alors aux variations de structures de Hodge (définies sur $\mathbb{R}$ ou $\mathbb{Q}$ ) et sa cohomologie calcule la cohomologie absolue au sens de A. Beilinson et P. Deligne. Il doit aussi exister des versions Hodge mixtes de ces constructions, controlant les variations de structures de Hodge mixtes et leur cohomologie (y compris pour des variétés ouvertes singulières).

3.5.2. Type d'homotopie (iso-)cristallin. Dans [40], M. Olsson construit des analogues cristallins des constructions présentées dans le paragraphe précédent. Dans le court paragraphe ci-dessous je résume la situation. Il va sans dire que les résultats de ce paragraphe sont tous dus à M. Olsson.

Dans [40], à une variété lisse et projective $X$ sur un corps algébriquement clos $k$ de caractéristique $p>0$, une sous-catégorie Tannakienne $\mathcal{C}$ de la catégorie $I \operatorname{soc}(X)$ des isocristaux sur $X$, et un point $x \in X(k)$, M. Olsson associe un type d'homotopie schématique pointé $X_{\mathcal{C}}$ sur $K=\operatorname{Frac}(W(k)$ ) (le corps des fractions de l'anneau des vecteurs de Witt sur $k$ ). Ce type d'homotopie schématique possède une catégorie des systèmes locaux en $K$-espaces vectoriels de dimension finie équivalente à la catégorie $\mathcal{C}$, et sa cohomologie à coefficients dans un tel système local est naturellement isomorphe à la cohomologie cristalline de $X$ à coefficients dans l'isocristal correspondant. Lorsque $\mathcal{C}=I \operatorname{soc}(X)$, le champ $X_{\mathcal{C}}$ est noté $(X, x)^{i s o c}$, appelé le type d'homotopie (iso-)cristallin de $X$, et est l'analogue 
cristallin du type d'homotopie de de Rham présenté précedemment. Un autre exemple intéressant est celui où $\mathcal{C}$ est engendrée, comme catégorie Tannakienne sur $K$, par les isocristaux provenant de $F$-isocrystaux. Dans ce cas, le champ $X_{\mathcal{C}}$ est noté $(X, x)^{F-i s o c}$ et appelé le type d'homotopie $F$-(iso-)cristallin de $X$.

Il existe un isomorphisme naturel $F^{*}\left((X, x)^{i s o c}\right) \simeq(X, x)^{i s o c}$, où $F^{*}$ désigne l'image réciproque par le Frobenius. Cet isomorphisme est l'analogue cristallin de l'action de $\mathbb{C}^{\times}$sur la schématisation d'une variété complexe présentée précédemment. Cependant, tout comme dans le cas complexe l'action de $\mathbb{C}^{\times}$n'est pas une action algébrique, l'isomorphisme $F^{*}\left((X, x)^{i s o c}\right) \simeq(X, x)^{i s o c}$ ne peut pas s'interpréter raisonablement comme une structure de $F$-isocristal sur le champ $(X, x)^{i s o c}$.

En contre partie, le champ $(X, x)^{F-i s o c}$ est lui muni d'une structure de $F$ isocristal, et le morphisme $(X, x)^{i s o c} \longrightarrow(X, x)^{F-i s o c}$ est l'analogue du morphisme $(X \otimes \mathbb{C})^{s c h} \longrightarrow \bar{X}^{\mathbb{H}}$. On peut décrire la structure de $F$-isocristal sur $(X, x)^{F-i s o c}$ à l'aide de la notion de type d'homotopie schématique non-neutre ${ }^{2}$ de la façon suivante.

Notons $\mathbb{H}_{F}$ la gerbe affine $\left(\operatorname{sur} \mathbb{Q}_{p}\right.$ ) dual de la catégorie Tannakienne des $F$ isocristaux sur Speck (voir par exemple [43, VI §4.3]). Comme $k$ est algébriquement clos le lien de $\mathbb{H}_{F}$ est un schéma en groupes diagonalisable dont le groupe des caractères est $\mathbb{Q}$ (ceci correspond à la décomposition des $F$-isocristaux à l'aide des pentes). La structure de $F$-isocristal sur $(X, x)^{F-i s o c}$ peut s'interpréter, à travers la correspondence entre $F$-isocristal sur $S p e c k$ et représentations linéaires de $\mathbb{H}_{F}$, comme un type d'homotopie schématique (non-neutre car la gerbe $\mathbb{H}_{F}$ elle même est non-neutre) $F^{0}$ sur $\mathbb{Q}_{p}$ muni d'une action de la gerbe $\mathbb{H}_{F}$. Ce champ $\mathbb{H}_{F^{-}}$ équivariant s'insére dans une suite de fibrations de type d'homotopie schématiques (non pointés)

$$
F^{0} \longrightarrow\left[F^{0} / \mathbb{H}_{F}\right] \longrightarrow \mathbb{H}_{F} .
$$

Le champ du milieu est relié de très près au type d'homotopie étale p-adique de $X$. En effet, les systèmes locaux de $\mathbb{Q}_{p}$-espaces vectoriels de dimension fini sur $\left[F^{0} / \mathbb{H}_{F}\right]$ correspondent aux $F$-isocrystaux sur $X$, et incluent donc les représentations continues $p$-adiques de $\pi_{1}^{e t}(X, x)$ de dimension finies. De plus, pour un système local $L$ sur $\left[F^{0} / \mathbb{H}_{F}\right]$ associé à une représentation $p$-adique $V$, la cohomologie de $\left[F^{0} / \mathbb{H}_{F}\right]$ à valeurs dans $L$ s'identifie avec la cohomologie étale $p$-adique de $X$ à valeurs dans $L$. La relation précise entre $\left[F^{0} / \mathbb{H}_{F}\right]$ et le type d'homotopie étale $p$-adique de $X$ peut aussi s'expliciter et l'on peut montrer que $F^{0}$ muni de son action de $\mathbb{H}_{F}$ détermine celui-ci a équivalence près (voir [40] pour plus de détails. D'une certaine façon, le type d'homotopie étale $p$-adique de $X$ est la partie de pente 0 de $F^{0}$ ).

\footnotetext{
${ }^{2}$ Un type d'homotopie schématique (non-neutre) sur un corps $k$ est un préfaisceau simplicial sur $A f f / k$, tel qu'il existe une extension de corps $k \subset K$, et un point $* \in F(K)$ faisant de $F$ un type d'homotopie schématique pointé sur $K$. Ils sont considérés comme des champs sur $k$ (i.e. des objets de $H o(k))$.
} 
3.5.3. Type d'homotopie $l$-adique. Pour terminer je signale l'existence d'un type d'homotopie $l$-adique d'un schéma $X$ sur lequel $l$ est inversible. Il s'agit d'une construction pour laquelle il n'existe pas de références détaillées (on trouvera tout de même quelques lignes dans $[58,59]$ ), et je me contenterais donc de donner un bref apperçu des principales propriétés. S'il le désire, le lecteur pourra considérer que les lignes qui suivent décrivent une situation conjecturale.

On fixe un schéma $X$ et $x \in X(\bar{k})$ un point géométrique. On peut alors construire un type d'homotopie schématique pointé sur $\mathbb{Q}_{l},\left(X^{e t} \otimes \mathbb{Q}_{l}\right)^{\text {sch }}$, appelé le type d'homotopie l-adique de $X$ et jouissant des propriétés suivantes. Les systèmes locaux de $\mathbb{Q}_{l}$-espaces vectoriels de dimension finie sur $\left(X^{e t} \otimes \mathbb{Q}_{l}\right)^{\text {sch }}$ sont en correspondance avec les représentations $l$-adiques continues de $\pi_{1}^{e t}(X, x)$. De plus, pour un tel système local $L$, la cohomologie de $\left(X^{e t} \otimes \mathbb{Q}_{l}\right)^{\text {sch }}$ s'identifie naturellement avec la cohomologie de $X$ à valeurs dans le système local étale $l$-adique correspondant. Le champ $\left(X^{e t} \otimes \mathbb{Q}_{l}\right)^{\text {sch }}$ est une version l-adique de la schématisation de l'espace topologique sous-jacent à une variété algébrique complexe.

Considèrons maintenant le cas où $X$ est une variété sur un corps $k$, et soit $\bar{X}=X \times_{\text {Spec } k} S p e c \bar{k}$ son extension à la clotûre séparable de $k$. Le groupe de Galois $H=\operatorname{Gal}(\bar{k} / k)$ opère par fonctorialité sur le champ non-pointé $\left(\bar{X}^{\text {et }} \otimes \mathbb{Q}_{l}\right)^{\text {sch }}$. Cette action n'est pas continue, de la même façon que l'action de $\mathbb{C}^{\times}$donnant la décomposition de Hodge décrite précédemment n'est pas une action de $\mathbb{G}_{m}$. On peut cependant considérer un certain quotient $\left(\bar{X}^{e t} \otimes \mathbb{Q}_{l}\right)_{a r}^{\text {sch }} \operatorname{de}\left(\bar{X}^{e t} \otimes \mathbb{Q}_{l}\right)^{\text {sch }}$, sur lequel $H$ va opèrer de façon continue. Par définition, la catégorie des systèmes locaux sur $\left(\bar{X}^{e t} \otimes \mathbb{Q}_{l}\right)_{a r}^{s c h}$ est la sous-catégorie Tannakienne des systèmes locaux $l$-adiques sur $\bar{X}$ engendré par ceux qui proviennent de systèmes locaux $l$-adiques sur $X$. De plus, pour $L$ un tel système local la cohomologie de $\left(\bar{X}^{e t} \otimes \mathbb{Q}_{l}\right)_{a r}^{s c h}$ à coefficients dans $L$ s'identifie naturellement avec la cohomologie de $\bar{X}$ à valeurs dans le système local $l$-adique correspondant.

Il existe une action naturelle de $H=\operatorname{Gal}(\bar{k} / k)$ sur le type d'homotopie schématique non-pointé $\left(\bar{X}^{e t} \otimes \mathbb{Q}_{l}\right)_{a r}^{s c h}$, et cette action est continue en un sens adéquat (e.g. on doit avoir que l'action de $H$ induite sur les $\mathbb{Q}_{l}$-espaces vectoriels (linéairement compacts) $\pi_{i}\left(\left(\bar{X}^{e t} \otimes \mathbb{Q}_{l}\right)_{a r}^{s c h}\right)$ est continue pour la topologie $l$-adique). Lorsque $k$ est par exemple un corps de nombre, le champ $H$-équivariant $\left(\bar{X}^{e t} \otimes\right.$ $\left.\mathbb{Q}_{l}\right)_{a r}^{s c h}$ est un analogue arithmétique du champ $\bar{X}^{\mathbb{H}}$ muni de sa décomposition de Hodge, ou encore de $(X, x)^{F-I \text { soc }}$ muni de sa structure de $F$-isocristal (voir $§ 3.5 .1$ et $\$ 3.5 .2)$.

Par fonctorialité, un point rationnel $x \in X(k)$ induit un point fixe homotopique de $H$ sur $\left(\bar{X}^{e t} \otimes \mathbb{Q}_{l}\right)_{a r}^{s c h}$, ou en d'autres termes un morphismes de champs $H$-équivariants $* \longrightarrow\left(\bar{X}^{e t} \otimes \mathbb{Q}_{l}\right)_{a r}^{s c h}$ (on rappelle que l'ensemble simplicial des points fixes homotopiques d'une action de $H$ sur un champ $F$ peut-être défini comme $\mathbb{R} \underline{H o m}_{H}(*, F)$, où $\mathbb{R} \underline{H o m}_{H}$ fait référence à la catégorie de modèles des 
préfaisceaux simplicial munis d'une action de $H$ ). On obtient donc une application

$$
N A J: X(k) \longrightarrow \pi_{0}\left(\left(\left(\bar{X}^{e t} \otimes \mathbb{Q}_{l}\right)_{a r}^{s c h}\right)^{h H}\right),
$$

de l'ensemble des points $k$-rationnels de $X$ vers l'ensemble des composantes connexes des points fixes homotopiques de $H$. Cette application s'appelle l'application d'Abel-Jacobi l-adique non-abélienne. On justifie cette terminologie en remarquant qu'il existe un diagramme commutatif

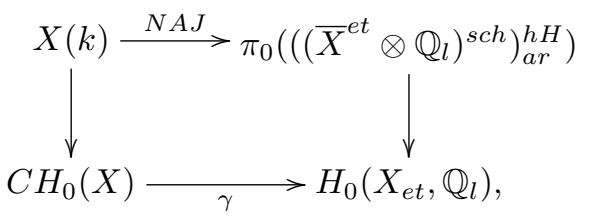

où $\gamma$ est le morphisme classe de cycle, et où le morphisme vertical de droite est induit par le morphisme d'abélianisation de $\left(\bar{X}^{\text {et }} \otimes \mathbb{Q}_{l}\right)_{a r}^{s c h}$ vers son type d'homologie. Notons que le morphisme classe de cycles permet de construire les applications d'Abel-Jacobi $l$-adiques supérieures (voir par exemple [27]), à l'aide de la suite spectrale de Leray-Serre (qui dégénerre en $E^{2}$ lorsque $X$ est propre et lisse)

$$
E_{p, q}^{2}=H_{\text {cont }}^{p}\left(H, H_{q}\left(\bar{X}_{e t}, \mathbb{Q}_{l}\right) \Rightarrow H_{q-p}\left(X_{e t}, \mathbb{Q}_{l}\right) .\right.
$$

L'existence de cette application $N A J$ semble montrer que le champ $\left(\bar{X}^{\text {et }} \otimes\right.$ $\left.\mathbb{Q}_{l}\right)_{a r}^{s c h}$, muni de son action du groupe $H$, détient des informations intéressantes sur les propriétés arithmétiques de $X$. Il serait par exemple intéressant de trouver des conditions sur la variété $X$ pour que l'application $N A J$ soit injective. On aimerait aussi trouver des exemples de variétés $X$ avec deux points $k$-rationnels $x$ et $y$, tel que $N A J(x) \neq N A J(y)$ mais $[x]=[y]$ dans le groupe de Chow des 0 cycles $\mathrm{CH}_{0}(X)$. Ceci impliquerait en particulier que l'application $N A J$ détecte des informations strictement plus fines que les applications d'Abel-Jacobi supérieures.

Remarquons enfin que lorsque $X$ est un courbe lisse projective de genre au moins 2 sur une corps de nombre $k$, l'application $N A J$ est une version $l$-adique de l'application déjà considérée par A. Grothendieck dans son programme de géométrie anabélienne, et qui à un point $k$-rationnel de $X$ associe un scindage de la suite exacte fondamentale

$$
1 \longrightarrow \pi_{1}^{e t}(\bar{X}) \longrightarrow \pi_{1}^{e t}(X) \longrightarrow H=\operatorname{Gal}(\bar{k} / k) \longrightarrow 1 \text {. }
$$

A. Grothendieck conjecture en particulier que cette dernière application est bijective. Il parrait donc raisonable d'essayer d'utiliser l'application $N A J$ afin de detecter les points rationnels de variétés arithmétiques qui ne sont plus des $K(\pi, 1)$ (comme par exemple des sections hyperplanes de $K(\pi, 1)$ ). J'espère revenir sur ce sujet dans un travail ultérieur. 


\section{Champs $\infty$-géométriques}

Dans cette section on supposera que $k$ est un corps. Cette hypothèse n'est nullement nécessaire mais simplifie quelque peu les définitions.

Rappelons que pour un espace vectoriel $V$ de dimension finie, on peut construire un champ algébrique (au sens d'Artin) $A l g_{V}$ classifiant les algèbres associatives et unitaires dont l'espace vectoriel sous-jacent est isomorphe à $V$. Rappelons aussi qu'étant donné une $k$-algèbre commutative $A$ de dimension fini on peut construire une variété projective Grass $_{A}$ dont les points classifient les chaines d'idéaux

$$
I_{n} \hookrightarrow I_{n-1} \ldots \hookrightarrow I_{0}=A .
$$

Dans ce paragraphe nous allons construire des champs de modules analogues à $\mathrm{Alg}_{V}$ et $\mathrm{Grass}_{A}$ dans le cadre de l'algèbre homotopique (i.e. lorsque $V$ est un complexe de $k$-vectoriel et $A$ une dga commutative, et que l'on ne s'intéresse aux structures qu'à quasi-isomorphismes près). Nous montrerons en particulier comment ces champs de modules sont recouverts par des champs affines pour en faire des champs $\infty$-géométriques.

\subsection{Définition}

On peut définir un champ algébrique (au sens d'Artin, voir par exemple [35]) comme le champ quotient associé à un groupoide affine et lisse (précisemment ceci ne donne que les champs algébriques quasi-compact et à diagonale affine, mais nous nous suffirons de ceci). Dans ce paragraphe nous allons généraliser la notion de champ algébrique en considérant des quotients associés à des objets en groupoides lisses (en un sens à définir) dans la catégorie des champs affines.

Pour commencer nous aurons besoin de certaines notions de finitude et de lissité pour des morphismes entre champs affines.

Soit $A \in k-A l g^{\Delta}$ une $k$-algèbre co-simpliciale (appartenant à $\mathbb{V}$ d'après nos conventions. On dispose de la catégorie $A / k-A l g^{\Delta}$, des objets sous $A$, munie de sa structure de catégorie de modèles induite (fibrations, équivalences et cofibrations testées dans $k-A l g^{\Delta}$ ), et dont les objets seront appelés des A-algèbres. La catégorie $A / k-A l g^{\Delta}$ possède une structure simpliciale (pour laquelle le foncteur d'oubli $A / k-A l g^{\Delta} \longrightarrow k-A l g^{\Delta}$ préserve les exponentiations par des ensembles simpliciaux) qui en fait une catégorie de modèles simpliciale. On peut donc définir des Hom's simpliciaux dérivés qui seront notés $\mathbb{R} \underline{\operatorname{Hom}}_{A-A l g}(-,-)$.

Définition 4.1.1. $\quad$ Soit $A \in k-A l g^{\Delta}$ une $k$-algèbre co-simpliciale et $B \in$ $A / k-A l g^{\Delta}$ une $A$-algèbre. Nous dirons que $B$ est de présentation finie sur $A$ (ou encore que le morphism $A \rightarrow B$ est de présentation finie) si pour tout système inductif filtrant de $A$-algèbres $\{C\}_{i \in I}$ (où $I \in \mathbb{V}$ ) le morphism naturel

$$
\operatorname{Colim}_{i \in I} \underline{\mathbb{H o m}}_{A-A l g}\left(B, C_{i}\right) \longrightarrow \mathbb{R} \underline{\operatorname{Hom}}_{A-A l g}\left(B, \operatorname{Colim}_{i \in I} C_{i}\right)
$$


est une équivalence.

- Un morphisme dans $H o\left(k-A l g^{\Delta}\right)$ est de présentation finie si l'on peut le représenter dans $k-A g^{\Delta}$ (à isomorphisme prés) par un morphisme de présentation finie au sens ci-dessus.

- Un morphisme de champs affines est de présentation finie s'il correspond par Cor. 2.2.3 à un morphisme de présentation finie dans $H o\left(k-A l g^{\Delta}\right)$.

Remarques:

- On remarque aisémment que si un morphisme de $H o\left(k-A l g^{\Delta}\right)$ possède un représentant dans $k-A l g^{\Delta}$ qui soit de présentation finie alors il en est de même de tous ses représentants. La notion de morphismes de présentation finie dans $H o\left(k-A l g^{\Delta}\right)$ est donc raisonable.

- Par adjonction on voit immédiatement qu'un morphisme de $k$-algèbres $A \longrightarrow$ $B$ (non co-simpliciales) est de présentation finie comme morphisme de $k-$ $A l g^{\Delta}$ si et seulement si $B$ est une $A$-algèbre de présentation finie au sens usuel. La définition 4.1.1 est donc une généralisation de la notion usuelle.

Nous passons maintenant à la notion de morphismes étales et de morphismes lisses.

Définition 4.1.2. Soit $f: F \longrightarrow G$ un morphisme de champs affines.

- Nous dirons que $f$ est formellement étale si pour toute $k$-algèbre $A \in k-A l g$, et $I \subset A$ un idéal de carré nul, le morphisme induit

$$
F(A) \longrightarrow F(A / I) \times_{G(A / I)}^{h} G(A)
$$

est une équivalence.

- Nous dirons que $f$ est étale s'il est formellement étale et de présentation finie

- Nous dirons que $f$ est un recouvrement étale s'il est étale et si de plus le morphisme de faisceaux $\pi_{0}(F) \longrightarrow \pi_{0}(G)$ est un épimorphisme.

Remarque: La notion de morphisme étale de champs affines généralise la notion usuelle pour les schémas affines. Cependant il est important de noter qu'il existe des recouvrements étales de champs affines $F \longrightarrow$ Spec $k$ où $F$ n'est pas représentable un schéma affine. En effet, on vérifie immédiatement que lorsque $k=\mathbb{F}_{p}$ le champ $K(\mathbb{Z} / p, 1)$ est un tel exemple.

Soit $E$ un $k$-espace vectoriel co-simplicial. Nous dirons que $E$ est parfait si $H^{*}(E)$ est de dimension finie sur $k$.

Rappelons que pour $E$ un $k$-espace vectoriel co-simplicial on dispose de la $k$ algèbre co-simpliciale libre sur $E$ notée $S^{*}(E)$. Un morphisme dans $H o\left(k-A l g^{\Delta}\right)$ sera dit parfait s'il est isomorphe à un morphisme de la forme

$$
A \longrightarrow A \otimes_{k} S^{*}(E),
$$

où $E$ parfait. De part la propriété universelle satisfaite par $S^{*}(E)$ on voit qu'un morphisme parfait est toujours de présentation fini au sens de Def. 4.1.1. Par le plongement $\mathbb{R} S p e c$ de Cor. 2.2 .3 on étent la notion de morphismes parfaits au cas des morphismes de champs affines. 
Définition 4.1.3. Un morphisme de champs affines $f: F \longrightarrow G$ est lisse s'il est de présentation fini et s'il existe des champs affines $F^{\prime}$ et $G^{\prime}$ et un diagramme commutatif de champs

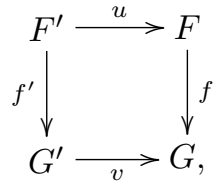

où $f^{\prime}$ est un morphisme étale, $u$ est un recouvrement étale, et $v$ est un morphisme parfait.

On vérifie que les morphismes lisses entre champs affines sont stables par composition et changements de bases (i.e. produits fibrés homotopiques). On vérifie aussi qu'un morphisme entre schémas affines est lisse au sens de la définition 4.1.3 si et seulement s'il est lisse au sens usuel.

Soit $X_{\bullet}: \Delta^{o p} \longrightarrow S \operatorname{Pr}(k)$ un objet simplicial dans la catégorie des préfaisceaux simpliciaux. Pour tout entier $n>1$ on dispose du $n$-éme morphisme de Segal (bien défini dans $H o(S P r(k))$ )

$$
\sigma_{n}: X_{n} \longrightarrow \underbrace{X_{1} \times_{X_{0}}^{h} X_{1} \cdots \times_{X_{0}}^{h} X_{1}}_{n \text { fois }},
$$

induit par les $n$ morphismes $[1] \longrightarrow[n]$ dans $\Delta$ qui envoient 0 et 1 sur $i$ et $i+1$ (pour $0 \leq i<n$ ). De même, on dispose du morphisme

$$
i: X_{2} \longrightarrow X_{1} \times_{X_{0}}^{h} X_{1},
$$

induit par les morphismes $d_{1}, d_{2}:[1] \longrightarrow[2]$, bien défini dans $H o(S \operatorname{Pr}(k))$. Lorsque tous les morphismes $\sigma_{n}$ et $i$ sont des équivalences nous dirons que $X$ • est un groupoide de Segal dans $\operatorname{SPr}(k)$.

Nous arrivons enfin à la définition des champs $\infty$-géométriques. La définition que nous donnons ici n'est pas la plus générale car elle n'englobe que le cas des quotients de champs affines par des champs en groupoides affines et lisses. Quoiqu'il en soit cette définition sera suffisante pour traiter les exemples qui nous intéressent dans cet article. Rappelons que pour $X_{\bullet}$ un objet simplicial de $S \operatorname{Pr}(k)$, en d'autres termes un préfaisceau bi-simplicial, nous notons $\left|X_{\bullet}\right|$ son préfaisceau simplicial diagonal. L'objet $\left|X_{\bullet}\right|$ est naturellement isomorphe dans $\operatorname{Ho}(\operatorname{SPr}(k))$ à la colimite homotopique du diagramme simplicial $n \mapsto X_{n}$.

Définition 4.1.4. Un champ $F$ est $\infty$-géométrique s'il est isomorphe à un champ de la forme $\left|X_{\bullet}\right|$, où $X_{\bullet}$ est un groupoide de Segal dans $\operatorname{SPr}(k)$ qui satisfait aux deux conditions suivantes.

- Les champs $X_{0}$ et $X_{1}$ sont des champs affines.

- Le morphisme $d_{0}: X_{1} \longrightarrow X_{0}$ est un morphisme lisse de champs affines. 
Il est immédiat de vérifier les propriétés suivantes.

- Tout champ affine est un champ $\infty$-géométrique.

- Un produits fibrés homotopique de champs $\infty$-géométriques est un champ $\infty$-géométrique. En particulier les champs $\infty$-géométriques sont stables par limites homotopiques finies.

- Un champ algébrique au sens d'Artin (voir [35]) qui est quasi-compact et dont la diagonale est un morphisme représentable et affine est un champ $\infty$-géométrique.

- Tout champ trés présentable au sens de Def. 3.2.5 est un champ $\infty$-géométrique.

La théorie des champs $\infty$-géométriques peut évidemment se poursuivre dans les mêmes directions que celles suivit par exemple dans [35, 53]. Nous ne le ferons pas ici et nous renvoyons à $[62,61]$ pour plus de détails sur une théorie plus générale.

\subsection{Application}

Dans ce paragraphe on présente un exemple d'application de la notion de champs $\infty$-géométrique du paragraphe précédent.

4.2.1. Le champ des structures multiplicatives. On fixe un complexe de $k$-espaces vectoriels $E$, à cohomologie bornée et de dimension finie. On cherche à définir le champ classifiant les structures multiplicatives sur $E$ à quasi-isomorphisme près, ou en d'autres termes les $k$-algèbres différentielles graduées (associatives et unitaires par exemple) dont le complex sous-jacent est quasi-isomorphe à $E$.

Pour cela, on rappelle que pout toute anneau $A$ (associatif, commutatif, unitaire), il existe une structure de catégorie de modèles sur la catégorie $A-D G A$, des $A$-algèbres différentielles graduées (non bornées). Les équivalences (resp. les fibrations) pour cette structure sont les quasi-isomorphismes (resp. les épimorphismes). L'existence de cette structure de modèles est démontrée par exemple dans [45].

Pour toute $k$-algèbre $A \in k-A l g$, on définit une catégorie $\underline{A l g}_{E}(A)$ de la façon suivante. Ses objets sont les $A$-algèbres différentielles graduées $B \in A-$ $D G A$, cofibrantes pour la structures de modèles décrite dans [45], et telles qu'il existe un morphisme fidèlement plat $A \longrightarrow A^{\prime}$ tel que les complexe de $A^{\prime}$-modules $B \otimes{ }_{A}^{\mathbb{L}} A^{\prime}$ et $E \otimes_{k}^{\mathbb{L}} A^{\prime}$ soient quasi-isomorphes. Les morphismes dans $A l g{ }_{E}(A)$ sont les équivalences (i.e. les quasi-isomorphismes) de $A$-algèbres différentielles graduées.

Pour un morphisme de $k$-algèbres $A \rightarrow A^{\prime}$, on dispose d'un foncteur de changement de base

$$
\underset{B}{\operatorname{Alg}_{E}(A)} \longrightarrow \frac{A l g_{E}}{B}\left(A^{\prime}\right)
$$

qui est bien défini car l'on s'est restreint aux $A$-algèbres différentielles graduées cofibrantes, et donc plates sur $A$ (ainsi le foncteur ci-dessus préserve bien les 
équivalences). Ceci définit un pseudo-foncteur

$$
\begin{array}{ccc}
\underline{A l g}_{E}: \quad k-A l g & \longrightarrow & C a t \\
A & \mapsto \quad & \underline{A l g}{ }_{E}(A),
\end{array}
$$

que l'on s'empresse de strictifier en un vrai foncteur par le procédé standard (voir par exemple [38, Thm. 3.4]).

Finalement, on compose avec le foncteur associant à toute catégorie son nerf pour obtenir un préfaisceau simplicial

$$
\begin{aligned}
& \underline{A l g}_{E}: \quad k-A l g \quad \longrightarrow \quad S \text { Set } \\
& A \quad \mapsto \quad B \underline{A l g}_{E}(A),
\end{aligned}
$$

et donc un objet de $S \operatorname{Pr}(k)$. Il se trouve que $B A \underline{A l g}_{E}$ ainsi défini est un champ au sens de Def. 1.1.3. La preuve de ce fait, qui semble cependant un résultat folklorique, est longue et technique (elle suit la démarche adoptée dans [23] pour démontrer que le préchamp des complexes est un champ) et nous ne la donnerons pas. Le théorème suivant donne un premier exemple non-trivial de champ $\infty$ géométrique. Nous nous contenterons d'esquisser sa preuve qui sera reprise en plus grande généralité dans [61].

Théorème 4.2.1. Le champ $B \underline{A l g}{ }_{E}$ est un champ $\infty$-géométrique.

Esquisse de preuve: Nous allons montrer que $B \underline{A l g} \underline{g}_{E}$ est en réalité le champ quotient d'un champ affine par un champ en groupes affine et lisse.

On commence par définir un champ $B \underline{\operatorname{Mod}}_{E}$ de la façon suivante. Pour toute $k$-algèbre $A$ on définit une catégorie $\underline{\operatorname{Mod}}_{E}(A)$, dont les objets sont les complexes de $A$-modules plats qui sont localement, pour la topologie plate sur $A$, quasiisomorphes à $E \otimes_{k} A$, et les morphismes sont les quasi-isomorphismes de complexes de $A$-modules. Lorsque $A$ varie dans $k-A l g$, ceci définit un pseudo-foncteur $A \mapsto$ $\underline{\operatorname{Mod}}_{E}(A)$ de $k-A l g$ vers $C a t$ que l'on strictifie et à qui l'on applique le foncteur classifiant $B: C a t \longrightarrow S S e t$. Ceci nous donne le champ cherché $B \underline{M o d}_{E}$.

Bien entendu, il existe un morphisme de champs $B \underline{A l g}_{E} \longrightarrow B \underline{M o d}_{E}$, qui oubli la structure d'algèbre différentielle graduée. De plus, $\overline{\operatorname{par}}[14,2.3]$ on voit que le champ $B \underline{\operatorname{Mod}}_{E}$ est isomorphe à $K(H, 1)$, où $H$ est le préfaisceau en monoides simpliciaux défini par

$$
\begin{array}{ccc}
H: k-A l g & \longrightarrow & \text { SMon } \\
A & \mapsto & A u t_{C(A)}\left(E \otimes_{k} A\right),
\end{array}
$$

où $A u t_{C(A)}\left(E \otimes_{k} A\right)$ désigne le sous-ensemble simplicial du l'ensemble simplicial des morphismes ("mapping spaces") $\operatorname{Map}_{C(A)}\left(E \otimes_{k} A, E \otimes_{k} A\right)$ de la catégorie de modèles des complexes (non bornés) de $A$-modules (voir par exemple $[19,26]$ pour la description de la structure de modèles $C(A)$ et la définition des mapping spaces) formé des quasi-isomorphismes.

Lemme 4.2.2. Le champ $H$ est affine et lisse. 
Preuve: On commence par considèrer le champ des endomorphismes de $E$

$$
\begin{aligned}
& \mathbb{R} \underline{\operatorname{End}}(E): \quad k-A l g \longrightarrow \quad \text { SSet } \\
& A \quad \mapsto \quad \operatorname{Map}_{C(A)}\left(E \otimes_{k} A, E \otimes_{k} A\right) .
\end{aligned}
$$

Il est facile de voir que ce champ est isomorphe à $\mathbb{R} \operatorname{Spec} S^{*}(M)$, où $M$ est le $k$-module co-simplicial associé au complexe tronqué $\tau_{\geq 0} E \otimes_{k} E^{*}$ par la correspondence de Dold-Puppe. Ainsi, le champ $\mathbb{R} \underline{\operatorname{End}}(E)$ est affine.

On regarde maintenant le sous-préfaisceau en monoides simpliciaux $H$ de $\mathbb{R} \underline{E n d}(E)$ formé des quasi-isomorphismes. Il existe un diagramme homotopiquement cartésien de champs

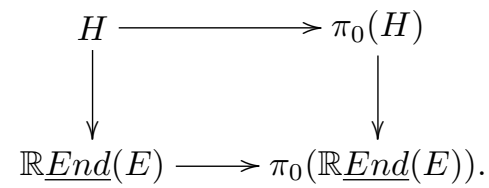

De plus, le faisceau en monoides $\pi_{0}(\mathbb{R} \underline{E n d}(E))$ est isomorphe au produit $\prod_{i} \mathcal{M}_{n_{i}}$, oú $\mathcal{M}_{n_{i}}$ est le schéma affine des matrices carées de rangs $n_{i}$, et $n_{i}=\operatorname{dim} H^{i}(E)$. De méme, $\pi_{0}(H)$ est le sous-faisceau des éléments inversibles dans $\pi_{0}(\mathbb{R} \underline{E n d}(E)$ ), ou en d'autres termes est isomorphe au produit $\prod_{i} G l_{n_{i}}$. Le diagrame homotopiquement cartésien précédent montre donc que $H$ s'écrit comme un produit fibré homotopique de champs affines et de présentation finie ( sur Speck) et donc est lui-même un champ affine et de présentation finie.

Il nous reste a voir que $H \longrightarrow S p e c k$ est lisse au sens de la définition Def. 4.1.3. Or, nous avons vu que le morphisme $\mathbb{R} \underline{\operatorname{End}}(E) \longrightarrow$ Speck est un morphisme parfait et donc lisse. Enfin, il est immédiat de vérifier que le morphisme naturel $H=\mathbb{R} \underline{A u t}(E) \longrightarrow \mathbb{R} \underline{E n d}(E)$ est étale au sens de la définition Def. 4.1.2, et donc lisse. Ceci montre que $H$ est un champ affine lisse.

On vient donc de construire un morphisme de champs

$$
B \underline{A l g}_{E} \longrightarrow K(H, 1),
$$

où $H$ est un préfaisceau en monoides simpliciaux dont le champ sous-jacents est affine et lisse. De plus, par définition le faisceau en monoides $\pi_{0}(H)$ est un faisceaux en groupes (i.e. $H$ est un $H_{\infty}$-champs au sens de Def. 1.4.2. On sait alors (voir par exemple [57, Prop. 1.5]) que $H$ est équivalent, en tant que préfaisceau en monoides simpliciaux, à un préfaisceau en groupes simpliciaux. On peut alors appliquer les techniques de champs équivariants, développées dans [31], qui nous apprennent que le champ $B A g_{E}$ est le champ quotient $[X / H]$, où $X$ est la fibre homotopique du morphisme $\overline{B A l g} E \longrightarrow K(H, 1)$. Le champ quotient $[X / H]$ s'écrit aussi de la forme $\left|X_{*}\right|$, où $X_{*}$ est le groupoide classifiant de l'action de $H$ sur $X$, défini par la formule $X_{n}:=X \times H^{n-1}$. Ainsi, le lemme 4.2.2 montre que pour démontrer le théorème 4.2.1 il nous suffit de voir que $X$ est un champ affine. 
Lemme 4.2.3. Le champ $X$, fibre homotopique du morphisme

$$
\underline{B A l g}_{E} \longrightarrow B \underline{\operatorname{Mod}}_{E}
$$

est un champ affine.

Preuve: Ce lemme est une conséquence d'un des résultats principal de [42].

On rappelle qu'il existe une structure de catégorie de modèles sur la catégorie des opérades (unitaires) dans la catégorie monoidale $C(k)$ des complexes $k$-espaces vectoriels (voir par exemple [19]). Pour toute $k$-algèbre $A \in k-A l g$ on définit une opérade $\underline{E n d}(E)_{A}$ dans $C(k)$ par la formule

$$
\underline{\operatorname{End}}(E)_{A}(n):=\underline{\operatorname{Hom}}_{k}\left(E^{\otimes_{k} n}, E \otimes_{k} A\right),
$$

où $\underline{H o m}_{k}$ désigne les Hom internes de la catégorie des complexes de $k$-espaces vectoriels. Pour un morphisme de $k$-algèbres $A \longrightarrow A^{\prime}$ on dispose d'un morphisme d'opérades $\underline{E n d}(E)_{A} \longrightarrow \underline{E n d}(E)_{A^{\prime}}$, et ceci fait de $A \mapsto \underline{E n d}(E)_{A}$ un préfaisceau en opérades unitaires sur la catégorie $A f f / k$.

On définit alors un foncteur

$$
\begin{array}{ccc}
\mathbb{R} \underline{\operatorname{Hom}}(\mathcal{A S S}, \underline{\operatorname{End}}(E)): \quad k-A l g & \longrightarrow & S S e t \\
A & \mapsto & \operatorname{Map}_{O p(k)}\left(\mathcal{A S S}, \underline{\operatorname{End}}(E)_{A}\right),
\end{array}
$$

où $M a p_{O p(k)}$ désigne les mapping spaces de la catégorie de modèles des opérades unitaires dans $C(k)$ (voir par exemple $[26, \S 5.2]$ ), et $\mathcal{A S S}$ est l'opérade unitaire finale (i.e. celle qui classifie les algèbres associatives et unitaires). On obtient ainsi un objet $\mathbb{R} \underline{\operatorname{Hom}}(\mathcal{A S S}, \underline{\operatorname{End}}(E)) \in \operatorname{Ho}(\operatorname{SPr}(k))$. Le théorème [42, Thm. 1.1.5] (ou du moins sa version où la catégorie de modèles $\mathcal{M}_{R}$ est rempmlacée par $\left.C(k)\right)$ nous dit que le champ $X$ est isomorphe au champ $\mathbb{R} \underline{H o m}(\mathcal{A S S}, \underline{E n d}(E))$. Il nous reste donc à montrer que le champ $\mathbb{R} \underline{H o m}(\mathcal{A S S}, \underline{\operatorname{End}}(E))$ est affine.

Pour cela, on écrit $\mathcal{A S S} \simeq \operatorname{Hocolim}_{n \in \Delta^{o p}} \mathcal{O}_{n}$, où chaque opérade $\mathcal{O}_{n}$ est une opérade libre. Ainsi, on a une équivalence naturelle de champs

$$
\mathbb{R} \underline{\operatorname{Hom}}(\mathcal{A S S}, \underline{\operatorname{End}}(E)) \simeq \operatorname{Holim}_{n \in \Delta} \mathbb{R} \underline{\operatorname{Hom}}\left(\mathcal{O}_{n}, \underline{\operatorname{End}}(E)\right),
$$

où $\mathbb{R} \underline{H o m}\left(\mathcal{O}_{n}, \underline{\operatorname{End}}(E)\right)$ est défini comme $\mathbb{R} \underline{\operatorname{Hom}}(\mathcal{A S S}, \underline{\operatorname{End}}(E))$ en remplaçant l'opérade $\mathcal{A S S}$ par $\mathcal{O}_{n}$. Par la proposition Prop. 2.2.7 il nous faut donc montrer que pour une opérade libre $\mathcal{O}$ le champ $\mathbb{R} \underline{\operatorname{Hom}}(\mathcal{O}, \underline{\operatorname{End}}(E))$ est un champ affine. Mais dire que $\mathcal{O}$ est libre signifie qu'il existe une famille de complexes $D_{n}$ pour $n \geq 1$ et des équivalences fonctorielles en $A$

$$
\begin{gathered}
\mathbb{R} \underline{\operatorname{Hom}}(\mathcal{O}, \underline{\operatorname{End}}(E))(A) \simeq \prod_{n} \operatorname{Map}_{C(k)}\left(E^{\otimes_{k} n} \otimes_{k} D_{n}, E \otimes_{k} A\right) \\
\simeq \prod_{n} \operatorname{Map}_{C(k)}\left(E^{\otimes_{k} n} \otimes_{k} E^{*} \otimes_{k} D_{n}, A\right),
\end{gathered}
$$

où $M a p_{C(k)}$ sont les mapping spaces de la catégorie de modèles des complexes de $k$-espaces vectoriels. Ceci montre qu'il suffit de vérifier que pour un complexe fixé $C$, le champ défini par $A \mapsto \operatorname{Map}_{C(k)}(C, A)$ est un champ affine. Mais il est clair que ce champ est isomorphe à $\mathbb{R} S p e c S^{*}(M)$, où $M$ est le $k$-espace vectoriel co-simplicial obtenu en appliquant la correspondence de Dold-Puppe au complexe 
tronqué $\tau_{\geq 0} C$.

En conclusion, $B \underline{A l g}_{E}$ s'écrit $[X / H]$, où $X$ est un champ affine et $H$ est un champ en groupes affine et lisse qui opère sur $X$. Ceci montre que $B \underline{A l g}{ }_{E}$ est un champ $\infty$-géométrique au sens de la définition 4.1.4.

\section{Remarques:}

- On pourrait aussi démontrer un théorème analogue au théorème Thm. 4.2.1 pour le champs classifiants les sturctures multiplicatives associatives, unitaires et commutatives. Dans le cas où $k$ n'est pas de caratéristique nulle il faut cependant utiliser la notion de $E_{\infty}$-algèbres au lieu de celle d'algèbres différentielles graduées commutatives.

- Des espaces de modules formels de structures mutiplicatives avaient déjà été construits dans $[33,20]$, et notre champ $B \underline{A l g} \underline{G}_{E}$ en est une contre-partie globale et tronquée. La version non-tronquée, ou encore dérivée du champ $B \underline{A l g}_{E}$ est définie et étudiée dans [62] (voir aussi [61]).

4.2.2. Périodes non-abéliennes. On suppose maintenant que $k=\mathbb{C}$. Pour une variété lisse projective complexe $X$, on dispose de sa $\mathbb{C}$-algèbre différentielle graduée commutative de cohomologie $C^{*}\left(X^{t o p}, \mathbb{C}\right) \simeq C^{*}\left(X, \Omega_{X}^{*}\right)$. Elle est muni d'une filtration de Hodge

$$
F^{i} C^{*}\left(X, \Omega_{X}^{*}\right):=C^{*}\left(X, \Omega_{X}^{>i}\right)
$$

qui est une filtration par des idéaux différentiels gradués. Dans cette section on se propose d'étudier la variation de la filtration de Hodge sur $C^{*}\left(X^{t o p}, \mathbb{C}\right)$ lorsque l'on déforme la variété $X$, et ce à l'aide d'une application des périodes non-abéliennes. L'approche que nous proposons dans ce paragraphe n'est qu'un première approximation et ne tient pas compte de toute la structure (en particulier la transversalité de Griffiths est négligée), et demandera certainement à être modifiée et completée dans un travail ultérieur.

On rappelle que pour toute $\mathbb{C}$-algèbre $B \in \mathbb{C}-A l g$, il existe une structure de catégorie de modèles sur la catégorie $B-C D G A_{+}$, des $\mathbb{C}$-algèbres différentielles graduées commutatives concentrées en degrés positifs, où les équivalences sont les quasi-isomorphismes et les fibrations sont les surjections (voir par exemple [4] ou encore la preuve de Thm. 2.1.2). On fixe un objet $A \in \mathbb{C}-C D G A_{+}$à cohomologie bornée et de dimension finie. Nous allons définir un champ $B \underline{F i l t}_{A}^{n}$, classifiant les filtrations de longueur $n$ sur $A$.

Pour $B \in \mathbb{C}-A l g$, on définit une catégorie $\underline{F i l t}_{A}^{n}(B)$ de la façon suivante. Les objets de $\underline{F i l t}_{A}^{n}(B)$ sont les diagrames dans $B-C D G A_{+}$

$$
A \otimes_{\mathbb{C}} B=A^{(n+1)} \longrightarrow A^{(n)} \longrightarrow \ldots A^{(1)} \longrightarrow A^{(0)}=0,
$$

vérifiant les deux conditions suivantes

- Pour tout entier $i \leq n$, l'objet $A^{(i)} \in B-C D G A_{+}$est cofibrant. 
- Pour tout entier $i$ le morphisme induit

$$
H^{*}\left(A \otimes_{\mathbb{C}} B\right) \simeq H^{*}(A) \otimes_{\mathbb{C}} B \longrightarrow H^{*}\left(A^{(i)}\right)
$$

est un épimorphisme scindé de $B$-modules (en particulier, on voit que tous les $B$-modules $H^{*}\left(A^{(i)}\right)$ sont projectifs et de type fini, et tous les morphismes $H^{*}\left(A^{(i)}\right) \longrightarrow H^{*}\left(A^{(i-1)}\right)$ sont des épimorphismes scindés de $B$-modules). $C D G A_{+}$

Les morphismes dans $\underline{F i l t}_{A}^{n}(B)$ sont les diagrames commutatifs dans $B-$

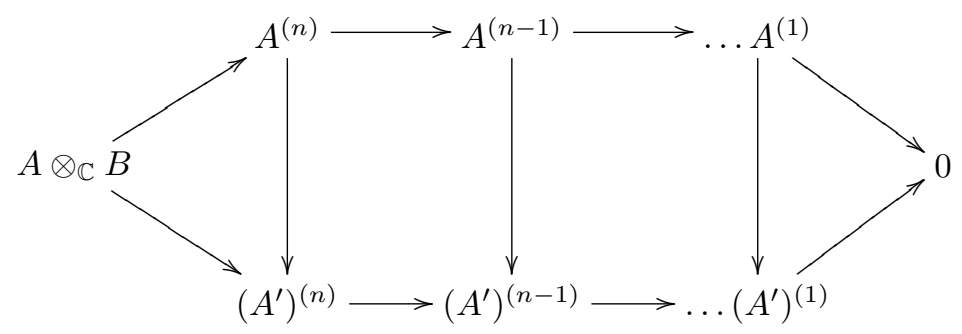

où les morphismes verticaux sont tous des quasi-isomorphismes.

Pour un morphisme de $\mathbb{C}$-algèbres $B \longrightarrow B^{\prime}$ on dispose d'un foncteur de changement de base $\underline{F i l t}_{A}^{n}(B) \longrightarrow \underline{F i l t}_{A}^{n}\left(B^{\prime}\right)$, qui à un objet

$$
A \otimes_{\mathbb{C}} B=A^{(n+1)} \longrightarrow A^{(n)} \longrightarrow \ldots A^{(1)} \longrightarrow A^{(0)}=0,
$$

associe l'objet

$$
A \otimes_{\mathbb{C}} B^{\prime}=A^{(n+1)} \otimes_{\mathbb{B}} B^{\prime} \longrightarrow A^{(n)} \otimes_{\mathbb{B}} B^{\prime} \longrightarrow \ldots A^{(1)} \otimes_{B} B^{\prime} \longrightarrow 0 .
$$

Ceci définit un pseudo-foncteur $B \mapsto \underline{F i l t}_{A}^{n}(B)$, que l'on strictifie et au quel on applique le foncteur classifiant pour obtenir un objet $B \underline{F i l t}_{A}^{n} \in S \operatorname{Pr}(\mathbb{C})$.

La proposition suivante est une généralisation de l'existence des variétés Grassmaniennes. Nous ne la démontrerons pas.

Proposition 4.2.4. Le préfaisceau simplicial BFilt ${ }_{A}^{n}$ est un champ $\infty$-géométrique.

Supposons maintenant que $B$ soit une $\mathbb{C}$-algèbre locale Artinienne et $p$ : $\mathcal{X} \longrightarrow$ Spec $B$ un morphisme projectif et lisse. Sur le site Zariski de $\mathcal{X}$, on dispose d'un diagrame de faisceaux de $B-C D G A_{+}$

$$
\Omega_{\mathcal{X} / B}^{*} \longrightarrow \Omega_{\mathcal{X} / B}^{\leq n-1} \longrightarrow \Omega_{\mathcal{X} / B}^{\leq n-2} \quad \cdots \longrightarrow \Omega_{\mathcal{X} / B}^{\leq 0}=\mathcal{O}_{\mathcal{X}}
$$

où $\Omega_{\mathcal{X} / B}^{*}$ est le complexe de de Rham (algébrique) relatif du morphismes $p$. En utilisant une structure de modèles adéquate sur la catégorie des faisceaux de $B-$ $C D G A_{+}$(voir par exemple [31]) on peut prendre l'image directe dérivée de ce diagrame

$$
\mathbb{R} p * \Omega_{\mathcal{X} / B}^{*} \longrightarrow \mathbb{R} p_{*} \Omega_{\mathcal{X} / B}^{\leq n-1} \longrightarrow \mathbb{R} p_{*} \Omega_{\mathcal{X} / B}^{\leq n-2} \quad \cdots \longrightarrow \mathbb{R} p_{*} \mathcal{O}_{\mathcal{X}}
$$


qui est un diagrame dans $B-C D G A_{+}$.

En utilisant la connexion de Gauss-Manin on peut voir qu'il existe une équivalence (non canonique) dans $B-C D G A_{+}$

$$
\mathbb{R} p * \Omega_{\mathcal{X} / B}^{*} \simeq C^{*}\left(X^{\text {top }}, \mathbb{C}\right) \otimes_{\mathbb{C}} B,
$$

où $X:=\mathcal{X} \otimes_{B} B / m$ est la fibre spéciale de $p$ et $C^{*}\left(X^{\text {top }}, \mathbb{C}\right)$ son algèbre différentielle graduée de cohomologie. Ainsi, si l'on choisit une telle équivalence et si l'on pose $A=C^{*}\left(X^{t o p}, \mathbb{C}\right)$, on obtient un diagrame dans $B-C D G A_{+}$

$$
A \otimes_{\mathbb{C}} B \longrightarrow \mathbb{R} p_{*} \Omega_{\mathcal{X} / B}^{\leq n-1} \longrightarrow \mathbb{R} p_{*} \Omega_{\mathcal{X} / B}^{\leq n-2} \quad \cdots \longrightarrow \mathbb{R} p_{*} \mathcal{O}_{\mathcal{X}} .
$$

Enfin, quitte à prendre un remplacement cofibrant de ce diagrame, il est bien connu que ceci définit un point dans $B \underline{F i l t}_{A}^{n}(B)$. Ce point peut aussi se représenter par un morphisme de champs

$$
\text { Spec } B \longrightarrow B \underline{F i l t}_{A}^{n},
$$

qui est appelé application des périodes non abéliennes de la famille $\mathcal{X} \longrightarrow$ Spec $B$. Ce morphisme est bien défini à un isomorphisme non canonique près (qui dépend du choix de la trivialisation $\left.\mathbb{R} p * \Omega_{\mathcal{X} / B}^{*} \simeq C^{*}\left(X^{t o p}, \mathbb{C}\right) \otimes_{\mathbb{C}} B\right)$.

Pour terminer, signalons que la construction précédente possède aussi la version globale suivante.

Soit $p: X \longrightarrow S$ un morphisme projectif et lisse de $\mathbb{C}$-schémas séparés de type fini. On suppose que $S$ est connexe et on choisit un point fermé $s \in S$. On pose $A=C^{*}\left(X_{s}^{\text {top }}, \mathbb{C}\right)$, la $\mathbb{C}$-algèbre différentielle graduée de cohomologie de la fibre de $p$ en $s$.

On dipose sur l'espace $S^{\text {top }}$ d'un faisceau de $\mathbb{C}-C D G A_{+}, \mathbb{R} p_{*}^{\text {top }}(\mathbb{C})\left(p^{\text {top }}\right.$ est le morphisme d'espaces topologique $X^{t o p} \longrightarrow S^{\text {top }}$ ), qui est localement équivalent au faisceau constant de fibre $A$. Il est donc classifié par un morphisme de monoides simpliciaux (morphisme de monodromie)

$$
\rho: G \longrightarrow \mathbb{R} \underline{A u t}{\underline{\mathbb{C}-C D G A_{+}}}(A),
$$

où $G$ est le groupe simplicial des lacets de $S^{\text {top }}$ (i.e. un groupe simplicial tel que $B G$ soit équivalent au type d'homotopie de $S^{t o p}$ ). En d'autres termes le groupe simplicial $G$ opère sur $A$, et donc aussi sur le préfaisceau simplicial $B \underline{F i l t}_{A}^{n}$. On peut donc former le champ quotient (i.e. le quotient homotopique par $G$ ) $D:=$ $\left[B \underline{F i l t}_{A}^{n} / G\right]$. Le champ $D$, qui n'est plus $\infty$-géométrique (mais seulement un champ analytique $\infty$-géométrique, en un sens à définir), peut être appelé le domaine des périodes non-abéliennes de la famille $p$.

On peut alors construire un morphisme de champs analytiques

$$
P: S^{a n} \longrightarrow D^{a n}
$$

qui est une globalisation de l'application des périodes définies ci-dessus. Pour cela il nous faudrait parler du procédé d'analytification de champs, et construire $P$ en recollant des applications des périodes locales pour les quelles le faisceau $\mathbb{R} p_{*}^{t o p}(\mathbb{C})$ 
est équivalent au faisceau constant de fibre $A$. Nous ne le ferons pas dans cet article.

L'espoir est bien entendu que les applications des périodes non-abéliennes detectent des variations que l'on ne peut détecter à l'aide des variations de structures de Hodge sur la cohomologie ou même sur les groupes d'homotopie. On pose donc la question suivante.

Problème: Trouver un morphisme projectif et lisse $X \longrightarrow S$ où $S$ est simplement connexe et où l'application des périodes non-abéliennes n'est pas constante.

Dans [54], C. Simpson construit un exemple de famille $X \longrightarrow S$, avec $S$ simplement connexe, et pour laquelle la variation sur le type d'homotopie de Dolbeault de la fibre est infinitésimalement non triviale. Cet exemple fournit-il une réponse positive à la question précédente? 


\section{Appendix A. Le problème de la schématisation}

Dans son manuscript [18], A. Grothendieck aborde un problème qu'il appelle problème de la schématisation des types d'homotopie. Dans cet appendice je résume ce que j'ai personellement compris de cette question. Il s'agit bien entendu d'un point de vue personel et de ce fait subjectif.

Tout d'abord, pour tout schéma de base $S$ (on supposera que $S=$ Speck pour un anneau $k$ ), il doit exister un notion $d$ ' $\infty$-champs en groupoides sur $S$ (appelés par la suite simplement champs sur $S$, ou champs sur $k$ ). On demande que les champs sur $S$ soient des généralisations des faisceaux et des champs en groupoides (au sens de [35]), par exemple sur le grand site fpqc de $S$.

Si l'on suit le point de vue de A. Grothendieck, les champs sur $S$ doivent former une $\infty$-catégorie. De façon plus approximative on peut demander qu'il existe des notions de morphismes de champs et d'équivalences de champs, qui permettent alors de parler de la catégorie homotopique des champs sur $S$, notée $H o(S)$ (obtenue en inversant formellement les équivalences).

Enfin, on s'attend à ce que les constructions standards sur les faisceaux (limites, colimites, Hom internes, images inverses et directes ...) s'étendent en des constructions au niveaux des champs. Par exemple il doit exister une notion de limites et colimites homotopique de champs sur $S$. Une manière efficace d'obtenir l'existence de telles constructions est de demander de plus que les champs sur $S$ forment une catégorie de modèles dont $H o(S)$ en est la catégorie homotopique. Cependant, pour des raisons qui semblent personelles A. Grothendieck n'envisage pas dans [18] d'utiliser la théorie des catégories de modèles.

La théorie des préfaisceaux simpliciaux de [30, 28] donne évidemment un exemple d'une telle théorie des champs (voir [24] pour plus de détails sur la comparaison entre champs en groupoides et préfaisceaux simpliciaux).

Supponsons maintenant que l'on dispose d'une théorie des champs sur $S$ satisfaisant les attentes décrites ci-dessus. En utilisant l'existences de colimites homotopiques on peut définir une construction $A \mapsto B A$, qui fait d'un champ en groupes abéliens $A$, un nouveau champ en groupes abéliens $B A$ dont le champ des lacets est équivalents à $A$. Par itérations on obtient des champs d'EilenbergMacLane

$$
K\left(\mathbb{G}_{a}, n\right):=B\left(K\left(\mathbb{G}_{a}, n-1\right)\right) \quad K\left(\mathbb{G}_{a}, 0\right):=\mathbb{G}_{a},
$$

où $\mathbb{G}_{a}$ est le faisceau en groupes additif sur $S$.

Les champs $K\left(\mathbb{G}_{a}, n\right)$ semblent d'un importance capitale aux yeux de Grothendieck, car il les considèrent comme les exemples primitifs fondamentaux de types d'homotopie schématiques, dont la définition est un des problème central de [18]. Bien qu'il ne précise jamais ce que doivent être ces types d'homotopie schématiques, les champs $K\left(\mathbb{G}_{a}, n\right)$ en sont toujours des exemples. On peut aussi déduire des constructions proposées dans [18] que les types d'homotopie schématiques forment une sous-catégorie pleine de la catégorie des champs $H o(S)$ qui 
est stable par limites homotopiques (ou encore de façon plus restrictive on peut demander la stabilité par produits fibrés homotopiques uniquement, si l'on veut se restreindre aux objets de type fini).

Nous dirons donc qu'une sous-catégorie pleine $\mathcal{C}$ de $H o(S)$ est une catégorie de types d'homotopie schématiques sur $S$, si elle est stable par limites homotopiques et si elle contient les champs $K\left(\mathbb{G}_{a}, n\right)$.

On se donne maintenant une catégorie de types d'homotopie schématiques sur $S, \mathcal{C} \subset H o(S)$, au sens ci-dessus. L'existence d'une théorie raisonable des colimites homotopiques dans $H o(S)$ implique que la catégorie $H o(S)$ est enrichie dans la catégorie homotopique des ensembles simpliciaux $H o(S S e t)$. Ainsi, on peut définir un foncteur des sections globales

$$
\begin{array}{cl}
\mathbb{R} \Gamma: H o(S) & \longrightarrow H o(\text { SSet }) \\
F & \mapsto \underline{H o m}(*, F) .
\end{array}
$$

Par restriction on obtient un foncteur

$$
\mathbb{R} \Gamma: \mathcal{C} \longrightarrow H o(\text { SSet }) .
$$

On dira alors que la catégorie $\mathcal{C}$ vérifie les conditions du problème de la schématisation si le foncteur $\mathbb{R} \Gamma$ possède un adjoint à gauche. Nous noterons cet adjoint par

$$
-\otimes k: H o(S S e t) \longrightarrow \mathcal{C} \text {. }
$$

Par adjonction on déduit la formule de conservation de la cohomologie suivante, chère à $\mathrm{A}$. Grothendieck

$$
H^{n}(X, k) \simeq H^{n}\left(X \otimes k, \mathbb{G}_{a}\right):=\left[X \otimes k, K\left(\mathbb{G}_{a}, n\right)\right]_{H o(S)},
$$

pour tout ensemble simplicial $X$.

Soit $\mathcal{C} \subset H o(S)$ une catégorie de types d'homotopie schématiques vérifiant les conditions du problème de la schématisation. On suppose de plus que $S=S p e c k$ où $k$ est un corps. On rappelle que l'on note

$$
-\otimes k: H o(S S e t) \longrightarrow H o(S)
$$

l'adjoint à gauche du foncteur $\mathbb{R} \Gamma$. On peut alors énoncer le problème de la schématisation de la façon suivante.

Problème de la schématisation: Soit $k$ un corps de caractéristique nulle (resp. de caractéristique positive $p>0$ ). Trouver des catégories de types d'homotopie schématiques $\mathcal{C} \subset H o($ Speck) qui vérifient les conditions du problème de la schématisation au sens ci-dessus, et tel que le foncteur

$$
-\otimes k: H o(\text { SSet }) \longrightarrow \mathcal{C} \subset H o(\text { Speck })
$$

restreint aux types d'homotopie rationnels 1-connexes et de type fini (resp. aux types d'homotopie p-complets 1-connexes et de type fini) soit pleinement fidèle. 
Bien entendu, de part son énoncé le problème de la schématisation possède de nombreuses solutions, dont au moins une triviale lorsque $\mathcal{C}=H o(S)$ (et $k$ est algèbriquement clos). Nous avons montré dans la paragraphe $\S 2.3$ et $\S 2.5$ que les champs affines sur un corps $k$ forment une catégorie $\mathcal{C}$ qui donne un solution au problème précédent.

Pour finir, signalons que l'on peut énoncer des versions quelque peu modifiées du problème précédent, en considérant par exemples des champs pointés, ou encore des champs connexes et/ou pointés ... Nous laissons le soin au lecteur de modifier les définitions précédentes pour qu'elles soient adaptées à ces nouveaux contextes. Nous avons montré dans le paragraphe $\S 3.3$ que les types d'homotopie schématiques donnaient une solution au problème de la schématisation dans le cas pointé et connexe.

\section{Références}

[1] M. Artin, B. Mazur, Etale homotopy, Lecture Notes in Math. 100, Springer 1969.

[2] B. Blander, Local projective model structure on simpicial presheaves, K-theory $\mathbf{2 4}$ (2001), No. 3, $283-301$.

[3] A.K. Bousfield, The localization of spaces with respect to homology, Topology 14 (1975), $133-150$.

[4] A.K. Bousfield, V.K.A.M. Gugenheim, On PL DeRham theory and rational homotopy type, Memoirs of the Amer. Math. Soc. 179, (1976).

[5] A.K. Bousfield, D.M. Kan, Homotopy limits, completions and localisations, Lecture Notes in Math. 304, Springer-Verlag, Berlin, 1975.

[6] L. Breen, T. Ekedahl, Construction et propriétés de la sphère schématique, C.R. Acad. Sci. Paris Sér. 1 Math. 300 (1985), No. 19, 665-668.

[7] E.H. Brown, R.H. Szczarba, Rational and real homotopy theory with arbitrary fundamental groups, Duke Math. J. 71 (1993), 299 - 316.

[8] M. Burger, S. Mozes, Finitely presented simple groups and products of trees, C.R. Acad. Sci. Paris Sér. I Math. 324 (1997), 747 - 752.

[9] P. Deligne, Le groupe fondamental de la droite projective moins trois points, dans Galois groups over $\overline{\mathbb{Q}}$, Math. Sci. Res. Inst. Publ. 16, Springer 1989.

[10] P. Deligne, Catégories Tannakiennes, in Grothendieck Festschrift Vol. II, progress in math. 87, Birkhauser Boston 1990.

[11] M. Demazure, P. Gabriel, Groupes algébriques, Tome I, Masson \& Cie. Paris, NorthHolland publishing company, 1970.

[12] A. Dold, The homology of symmetric products and other functors of complexes, Ann. of Math. 68 (1958), $54-80$

[13] D. Dugger, S. Hollander, D. Isaksen, Hypercovers and simplicial presheaves, Math. Proc. Cambridge Philos. Soc. 136 (2004), 9-51.

[14] B. Dwyer, D. Kan, A classification theorem for diagramms of simplicial sets, Topology 23, (1984), 139-155. 
[15] P. Goerss, J.F. Jardine, Simplicial homotopy theory, Progress in Mathematics, Vol. 174, Birkhauser Verlag 1999.

[16] P. Goerss, Simplicial chains over a field and p-local homotopy theory, Math. Z. 220 (1995), $523-544$.

[17] S. Gomez-Tato, S. Halperin, D. Tanré, Rational homotopy theory for non-simply connected spaces, Trans. of the A.M.S. 352, No. 4 (1999), 1493 - 1525.

[18] A. Grothendieck, Pursuing stacks, munuscript non publié.

[19] V. Hinich, Homological algebra of homotopical algebras, Comm. in Algebra 25 (1997), 3291-3323.

[20] V. Hinich, Formal stacks as dg-coalgebras, J. Pure Appl. Algebra 162 (2001), No. 2-3, 209-250.

[21] V.A. Hinich, V.V. Schechtman, Homotopy limits of homotopy algebras, in K-theory, arithmetic and geometry, Lecture Notes in Mathematics 1289, Springer, Berlin $240-$ 264.

[22] P. Hirschorn, Localization of model categories, Mathematical Surveys and Monographs 99, AMS, 2003.

[23] A. Hirschowitz, C. Simpson, Descente pour les n-champs. Prépublication arXiv math.AG/9807049.

[24] S. Hollander, A homotopy theory for stacks, Prépublication arXiv math.AT/0110247.

[25] G. Hoshschild, G.D. Mostov, Representations and representative functions of Lie groups, Ann. Math, 66 (1957), 495 - 542.

[26] M. Hovey, Model categories, Mathematical surveys and monographs, Vol. 63, Amer. Math. Soc., Providence 1998.

[27] U. Jansen, Mixed motives and algebraic K-theory, Lecture Notes in Math. 1400.

[28] J. F. Jardine, Simplicial presheaves, J. Pure and Appl. Algebra 47 (1987), 35 - 87.

[29] J. F. Jardine, Stacks and the homotopy theory of simplicial sheaves, in Equivariant stable homotopy theory and related areas (Stanford, CA, 2000). Homology Homotopy Appl. 3 (2001), No. 2, 361-384.

[30] A. Joyal, Lettre à A. Grothendieck, 1984.

[31] L. Katzarkov, T. Pantev, B. Toën, Schematic homotopy types and non-abelian Hodge theory. Pré-publication math.AG/0107129.

[32] L. Katzarkov, T. Pantev, B. Toën, Some properties of the schematization functor. Pré-publication arXiv math.AG/0503418.

[33] M. Kontsevich, Y. Soibelman, Deformations of algebras over operads and the Deligne conjecture, Conférence Moshé Flato 1999, Vol. 1 (Dijon), 255-307, Math. Phys. Stud. 21, Kluwer Acad. Publ, Dordrecht, 2000.

[34] I. Kriz, p-adic homotopy theory, Topology and its applications 52 (1993), 279 - 308.

[35] G. Laumon and L. Moret-Bailly, Champs algébriques, A series of Modern Surveys in Mathematics vol. 39, Springer-Verlag 2000.

[36] A. Lubotzky, A. Magid, Varieties of representations of finitely generated groups, Memoirs of the Amer. Math. Soc. 336, (1985).

[37] M. Mandell, $E_{\infty}$-algebra and p-adic homotopy theory, Topology 40 (2001), no. 1, pp. 43-94. 
[38] J.P. May, Pairings of categories and spectra, JPAA 19 (1980), 299-346.

[39] J.S. Milne, Etale cohomology, Princeton University Press, Princeton, New Jersey 1980.

[40] M. Olsson, Crystalline structures on homotopy types associated with isocrystals. Prépublication disponible à http://www-math.mit.edu/ molsson.

[41] D. Quillen, Rational homotopy theory, Ann. of Math. 90 (1969), 205 - 295.

[42] C. Rezk, Spaces of algebra structures and cohomology of operads, Thèse (MIT), disponible à http://www.math.uiuc.edu/ rezk/papers.html.

[43] N. Saavedra, Categories Tannakiennes, Lecture Notes in Math. 265, Springer 1972.

[44] J. Sauloy, Théorie de Galois des équations aux q-différences fuschiennes, Thèse de l'université Paul Sabatier, Toulouse 1999.

[45] S. Schwede, B. Shipley, Algebras and modules in monoidal model categories, Proc. London Math. Soc. (3) 80 (2000), 491 - 511.

[46] G. Segal, Homotopy everything $H$-space, manuscipt.

[47] M. Demazure, A. Grothendieck, Schémas en groupes I: Propriétés générales des schémas en groupes, Lecture notes in Math. 151, Springer-Verlag 1970.

[48] M. Artin, A. Grothendieck, J.L. Verdier, Théorie des topos et cohomologie étale des schémas I, Lecture notes in Math. 269, Springer, Berlin 1972 - 1973.

[49] M. Artin, A. Grothendieck, J.L. Verdier, Théorie des topos et cohomologie étale des schémas II, Lecture notes in Math. 270, Springer, Berlin 1972 - 1973.

[50] C. Simpson, Higgs bundle and local systems, Publ. Math. I.H.E.S. 75 (1992), 5 - 95.

[51] C. Simpson, Homotopy over the complex numbers and generalized cohomology theory, dans Moduli of vector bundles (Taniguchi symposium December 1994, M. Maruyama ed., Dekker (1996), 229 - 263.

[52] C. Simpson, On a relative notion of algebraic Lie group and applications to $n$-stacks. Prépublication arXiv math.AG/9607002.

[53] C. Simpson, Algebraic n-stacks. Prépublication arXiv math.AG/9609014.

[54] C. Simpson, Secondary Kodaira-Spencer classes and non-abelian Dolbeault cohomology. Pré-publication math.AG/9712020.

[55] L. Smith, Homological algebra and the Eilenberg-Moore spectral sequence, Trans. Amer. Math. Soc. 129 (1967), 58 - 93.

[56] D. Sullivan, Infinitesimal computations in topology, Publ. Math. I.H.E.S. 47 (1978), $269-331$.

[57] B. Toën, Vers une interprétation galoisienne de la théorie de l'homotopie, Cahiers de Top. et Géom. Diff. Cat. 43 (2002), No. 4, 257-312.

[58] B. Toën, Dualité de Tannaka supérieure I: Structures monö̈dales. Pré-publication disponible à http://www.mpim-bonn.mpg.de.

[59] B. Toën, Homotopical and higher categorical structures in algebraic geometry, thèse d'habilitation, Mai 2003. Prépublication arXiv math.AG/0312262.

[60] B. Toën, G. Vezzosi, Homotopical algebraic geometry I: Topos theory, Advances in Mathematics, 193, Issue 2 (2005), p. 257-372.

[61] B. Toën, G. Vezzosi, Homotopical algebraic geometry II: Geometric stacks, à parraitre dans Memoirs of the Amer. Math. Soc.. Prépublication arXiv math.AG/0404373. 
[62] B. Toën, G. Vezzosi, From HAG to DAG: derived moduli spaces, à parraitre dans "Axiomatic, enriched and motivic homotopy theory", Proceedings of a NATO-ASI at the Isaac Newton Institute of Mathematical Sciences, 2002 (Workshop Organisers: J. Greenlees, R. Jardine, F. Morel). Prépublication arXiv math.AG/0210407.

Bertrand Toën

Laboratoire Emile Picard UMR CNRS 5580

Université Paul Sabatier, 118, route de Narbonne

31062 Toulouse Cedex 04, France

e-mail: toen@math.ups-tlse.fr 